\title{
IgA transcytosis and antigen recognition govern ovarian cancer immunity
}

https://doi.org/10.1038/s41586-020-03144-0

Received: 22 September 2019

Accepted: 17 December 2020

Published online: 3 February 2021

\section{Open access}

Check for updates

\begin{abstract}
Subir Biswas', Gunjan Mandal', Kyle K. Payne', Carmen M. Anadon', Chandler D. Gatenbee ${ }^{2}$, Ricardo A. Chaurio', Tara Lee Costich', Carlos Moran ${ }^{3}$, Carly M. Harro', Kristen E. Rigolizzo', Jessica A. Mine', Jimena Trillo-Tinoco', Naoko Sasamoto ${ }^{4}$, Kathryn L. Terry ${ }^{4}$, Douglas Marchion ${ }^{4}$, Andrea Buras ${ }^{5}$, Robert M. Wenham ${ }^{5}$, Xiaoqing Yu $^{6}$, Mary K. Townsend ${ }^{7}$, Shelley S. Tworoger ${ }^{7,8}$, Paulo C. Rodriguez ${ }^{1}$, Alexander R. Anderson ${ }^{2} \&$ Jose R. Conejo-Garcia ${ }^{1 凶}$
\end{abstract}

Most ovarian cancers are infiltrated by prognostically relevant activated $\mathrm{T}$ cells ${ }^{1-3}$, yet exhibit low response rates to immune checkpoint inhibitors ${ }^{4}$. Memory $\mathrm{B}$ cell and plasma cell infiltrates have previously been associated with better outcomes in ovarian cancer ${ }^{5,6}$, but the nature and functional relevance of these responses are controversial. Here, using 3 independent cohorts that in total comprise 534 patients with high-grade serous ovarian cancer, we show that robust, protective humoral responses are dominated by the production of polyclonal IgA, which binds to polymeric IgA receptors that are universally expressed on ovarian cancer cells. Notably, tumour B-cell-derived IgA redirects myeloid cells against extracellular oncogenic drivers, which causes tumour cell death. In addition, IgA transcytosis through malignant epithelial cells elicits transcriptional changes that antagonize the RAS pathway and sensitize tumour cells to cytolytic killing by T cells, which also contributes to hindering malignant progression. Thus, tumour-antigen-specific and -antigen-independent IgA responses antagonize the growth of ovarian cancer by governing coordinated tumour cell, $\mathrm{T}$ cell and $\mathrm{B}$ cell responses. These findings provide a platform for identifying targets that are spontaneously recognized by intratumoural B-cell-derived antibodies, and suggest that immunotherapies that augment $B$ cell responses may be more effective than approaches that focus on $T$ cells, particularly for malignancies that are resistant to checkpoint inhibitors.
Ovarian cancer is an immunogenic disease in which the pre-established immunoreactive landscape determines the outcome of the patient ${ }^{1-3,7}$. However, as monotherapies such as immune checkpoint inhibitors that augment $\mathrm{T}$ cell activity have only very modest response rates in patients with advanced disease ${ }^{4}$. Recent studies have suggested that plasma cell and memory B cell infiltrates, including those in tertiary lymphoid structures ${ }^{8}$, are associated with the cytolytic activity of T cells at ovarian cancer beds, resulting in improved survival of patients ${ }^{5,6}$. Although these studies suggest that humoral responses may potentiate $T$ cell immune surveillance, the roles of different antibody isotypes in malignant progression are controversial.

To characterize the role of B cells in ovarian cancer, we first stained a panel of 534 annotated high-grade serous ovarian cancers (HGSOCs) from 3 independent cohorts with $\mathrm{T}$ and $\mathrm{B}$ cell markers. As expected, $\mathrm{CD} 19^{+} \mathrm{B}$ cell infiltrates were associated with improved overall survival (Extended Data Fig. 1a, b), similar to data from The Cancer Genome Atlas (TCGA) (Extended Data Fig. 1c), and positively correlated with
T cell infiltrates (Extended Data Fig. 1d, e). In addition, intra-epithelial T cells only predict improved survival ${ }^{2}$ when $B$ cells co-infiltrate tumour islets (Extended Data Fig. 1f).

To characterize the isotypes produced by these B cells, we analysed viable single-cell suspensions from 29 freshly dissociated HGSOCs. Intracellular staining of plasma cells and $\mathrm{CD} 19^{+} \mathrm{CD} 20^{-} \mathrm{CD} 38^{\text {high }} \mathrm{CD} 27^{+}$ cells (defined as plasmablasts) revealed the dominant production of class-switched IgA, followed by IgG; this is consistent with mRNA expression data from TCGA (Fig. 1a, Extended Data Figs. 1g-i, 2a, b) and with surface staining of B cells (Fig. 1a, Extended Data Fig. 1g-i).

As previously reported ${ }^{6}, \mathrm{CD} 138^{+}$plasma cell infiltrates were associated with improved survival in patients with HGSOC (Extended Data Fig. 2 c), were identified in $80 \%$ of dissociated tumours in $>1 \%$ of total leukocytes and also correlated with intratumoural $\mathrm{T}$ cells (Extended Data Fig. 2d, e).

$\mathrm{CD}^{-} 5^{-} \mathrm{EpCAM}^{+}$tumour cells were also coated by IgA in all the dissociated HGSOCs we evaluated (Fig. 1b). Accordingly, we found universal expression of the polymeric immunoglobulin receptor (pIgR)

'Department of Immunology, H. Lee Moffitt Cancer Center and Research Institute, Tampa, FL, USA. ${ }^{2}$ Department of Mathematical Oncology, H. Lee Moffitt Cancer Center and Research Institute, Tampa, FL, USA. ${ }^{3}$ Department of Pathology, H. Lee Moffitt Cancer Center and Research Institute, Tampa, FL, USA. ${ }^{4} \mathrm{Obstetrics}$ and Gynecology Epidemiology Center, Brigham and

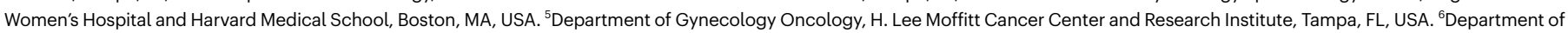
Biostatistics and Bioinformatics, H. Lee Moffitt Cancer Center and Research Institute, Tampa, FL, USA. ${ }^{7}$ Department of Cancer Epidemiology, H. Lee Moffitt Cancer Center and Research Institute, Tampa, FL, USA. ${ }^{8}$ Department of Epidemiology, Harvard T.H. Chan School of Public Health, Boston, MA, USA. ${ }^{凶}$ e-mail: Jose.Conejo-Garcia@moffitt.org 

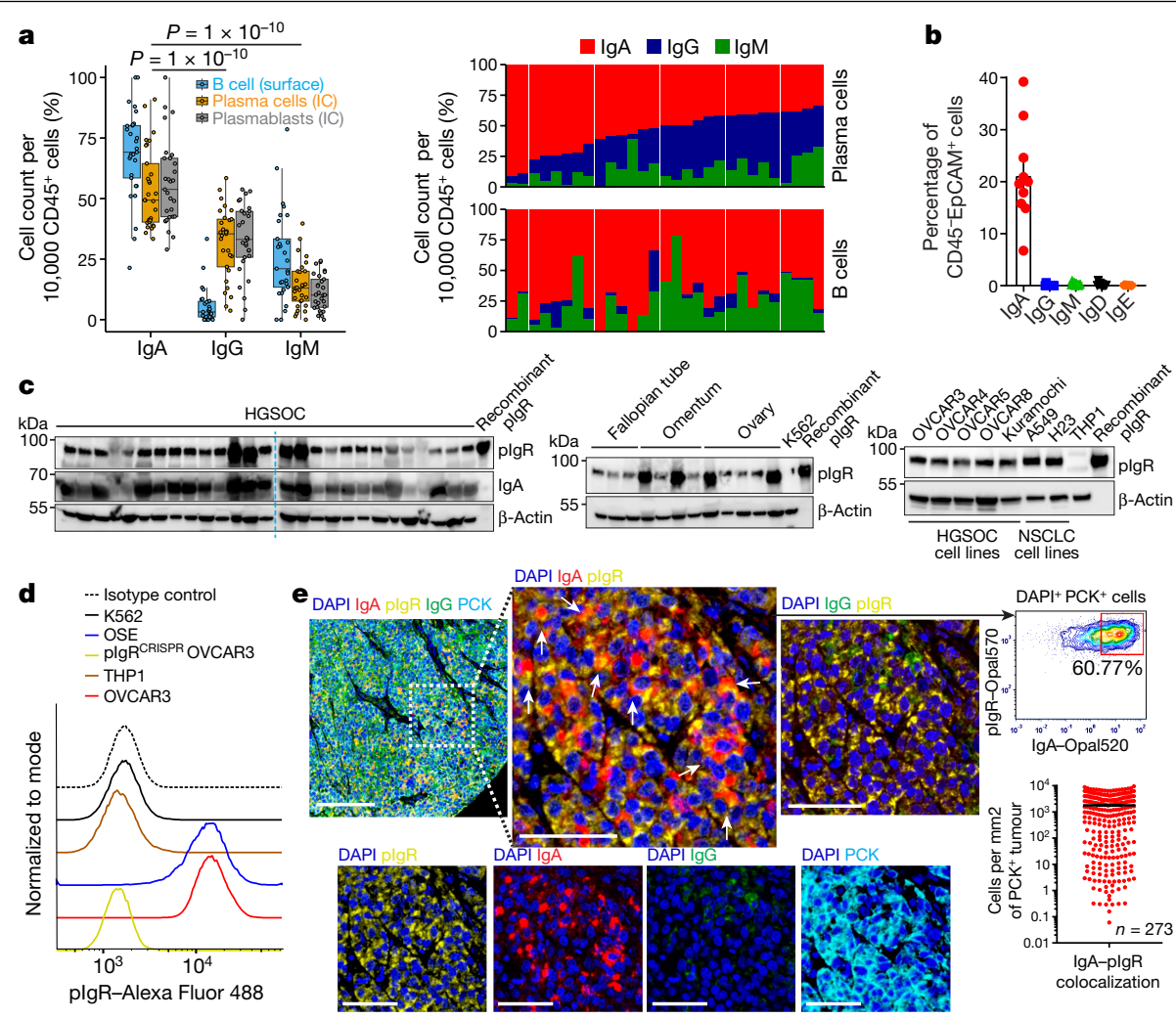

$\mathrm{DAPI}^{+} \mathrm{PCK}^{+}$cells
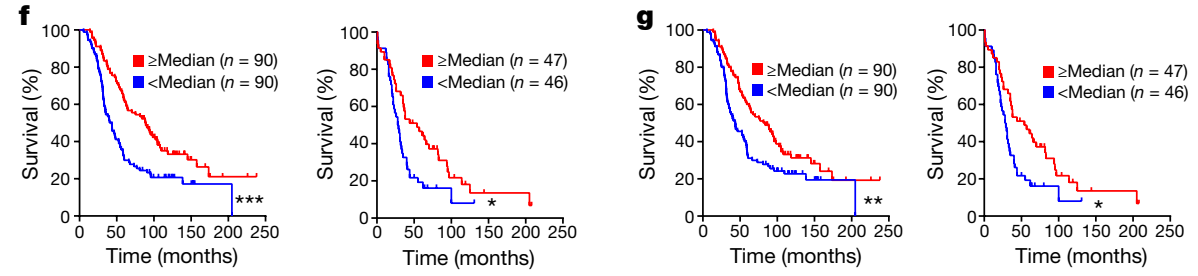

Fig. 1 IgA-plgR colocalization is associated with protective immunity in human ovarian cancer. a, Left, percentage of FACS cell counts of $\lg \mathrm{A}^{+}, \operatorname{IgG}^{+}$or $\operatorname{IgM}^{+}$cells among Ig $^{+} B$ cells or plasmablasts or plasma cells, normalized to 10,000 viable $\mathrm{CD} 45^{+}$cells. $\mathrm{B}$ cells, $\mathrm{CD} 45^{+} \mathrm{CD}^{-} \mathrm{CD} 19^{+} \mathrm{CD} 2 \mathrm{O}^{+}$cells; plasmablasts, $\mathrm{CD}^{\circ} 5^{+} \mathrm{CD}^{-} \mathrm{CD}^{-} 9^{+} \mathrm{CD} 20^{-} \mathrm{CD} 38^{\text {high }}$ cells; plasma cells, $\mathrm{CD} 45^{+} \mathrm{CD}^{-} \mathrm{CD}^{-} 9^{+} \mathrm{CD} 20^{-}$ $\mathrm{CD} 138^{+}$and $\mathrm{CD} 45^{+} \mathrm{CD} 3^{-} \mathrm{CD} 19^{-} \mathrm{CD} 20^{-} \mathrm{CD} 138^{+}$cells. Each dot represents one tumour $(n=29)$. Details of box plots can be found in Methods. $P$ values were obtained by a two-way analysis of variance (ANOVA) followed by Dunnett's test for multiple comparisons. Supplementary Table 1 provides further details on statistics. Right, bar graphs representing the percentage of each isotype produced by plasma cells (top) or B cells (bottom) in the same tumours, normalized to 10,000 viable $\mathrm{CD} 45^{+}$cells. IC, intracellular. b, IgA-coated $\mathrm{CD}^{-} 5^{-} \mathrm{EpCAM}^{+}$tumour epithelial cells (mean \pm s.e.m., $n=10$ ) in dissociated HGSOC.c, Expression of plgR protein in independent HGSOC $(n=27)$; tumour-free Fallopian tube $(n=3)$, ovary $(n=5)$ and omental $(n=4)$ samples; ovarian tumour cell lines; and K562 leukaemia cells and THP1 monocyte cells (negative controls). Positive control, recombinant human plgR. Western blots were repeated twice. NSCLC, non-small-cell lung cancer. d, Histograms

in HGSOC, as well as in tumour-free fallopian tube, ovarian and omental tissue (but not in THP1 monocytes or in K562 leukaemia cells) (Fig. 1c). Expression of cell-surface pIgR was confirmed by flow cytometry in cancer and ovarian surface cell lines (Fig.1d). Consequently, IgA and pIgR colocalized at tumour beds within cytokeratin (PCK)-positive tumour islets in 273 HGSOCs we analysed (Fig. 1e), and IgA-pIgR colocalization-but not pIgR-overexpression alone or stromal IgA-is associated with improved survival (Fig. 1f, Extended Data Fig. 2f, g). Importantly, the coating of tumour cells by IgA-but not IgG-is associated with improved survival (Fig. 1g, Extended Data Fig. 2h), and with increased intra-epithelial $\mathrm{CD}^{+}$and $\mathrm{CD}^{+} \mathrm{T}$ cells (Extended Data Fig. 3a).

showing FACS analysis of plgR, in ovarian surface epithelial (OSE), K562, THP1, wild-type or PIGR-ablated (pIgR ${ }^{\text {CRISPR }}$ ) OVCAR3 cells.e, Left, representative $(n=273)$ combined staining of IgA, plgR, IgG, PCK and DAPI. Instances with IgA-pIgR colocalization are indicated with arrows. Scale bar, $50 \mu \mathrm{m}$ (top left), $20 \mu \mathrm{m}$ (all other panels). Top right, representative ( $n=137$, IgA-plgR colocalization $\geq$ median) dot plot showing IgA-pIgR colocalized signal among $\mathrm{DAPI}^{+} \mathrm{PCK}^{+}$cells. Bottom right, scattered graph showing number of IgA-plgR colocalized cells (averaged from duplicated cores) per $\mathrm{mm}^{2}$ of $\mathrm{PCK}^{+}$ (mean \pm s.e.m., $n=273$ ). f, Increased numbers of cells with IgA-plgR colocalization per $\mathrm{PCK}^{+}$tumour islet area (averaged from duplicated cores) are associated with improved outcome (threshold, median; $P=0.0116$, H. Lee Moffett Cancer Centre cohort (MCC) (right); $P=0.0002$, New England CaseControl study cohort (NECC) (left)). g, Density of IgA-coated cells (averaged from duplicated cores) in tumour islets (cells per $\mathrm{mm}^{2} \mathrm{PCK}^{+}$area) is associated with improved outcome ( $P=0.0110$ for MCC (right) and $P=0.0054$ for NECC (left) cohorts). ${ }^{*} P \leq 0.05,{ }^{* *} P \leq 0.01,{ }^{* * *} P \leq 0.001$, two-sided log-rank (Mantel-Cox) test.

To determine whether IgA-pIgR interactions elicit transcytosis through tumour cells, we first incubated plgR ${ }^{+}$OVCAR3 ovarian cancer cells with fluorescently labelled non-antigen-specific IgA or IgG (Fig. 2a, Extended Data Fig. 3b). Confocal microscopy confirmed that IgA was selectively internalized and deposited on the cell surface within $8 \mathrm{~h}$ (Fig. 2a). Internalization was abrogated upon pepsin-mediated removal of the Fc or CRISPR-mediated ablation of PIGR (Fig. 2a, Extended Data Fig. 3b, c), and co-immunoprecipitation analyses of IgA and pIgR confirmed their physical interaction in human HGSOC (Extended Data Fig. 4a). In support of the notion that IgA indeed transcytoses through tumour cells, several peptides of the 


\section{Article}

a
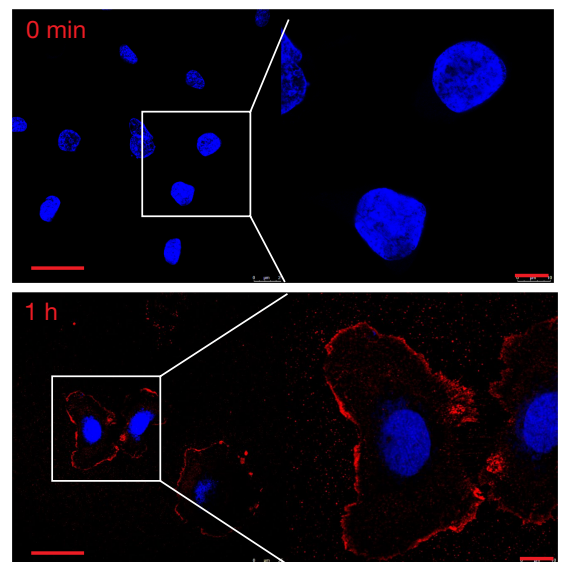

IgA-APC DAPI
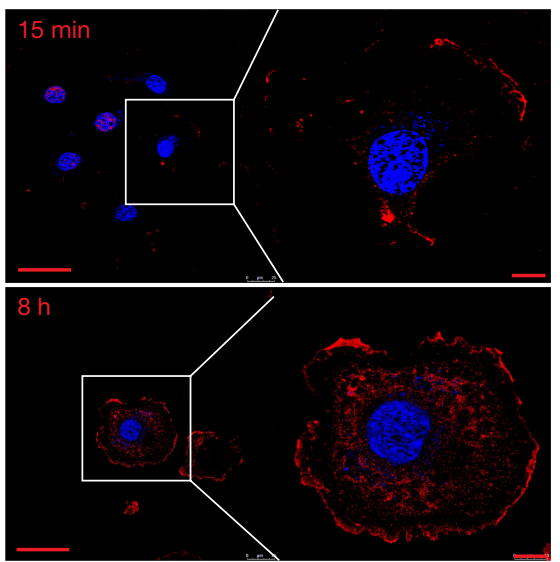

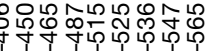

b

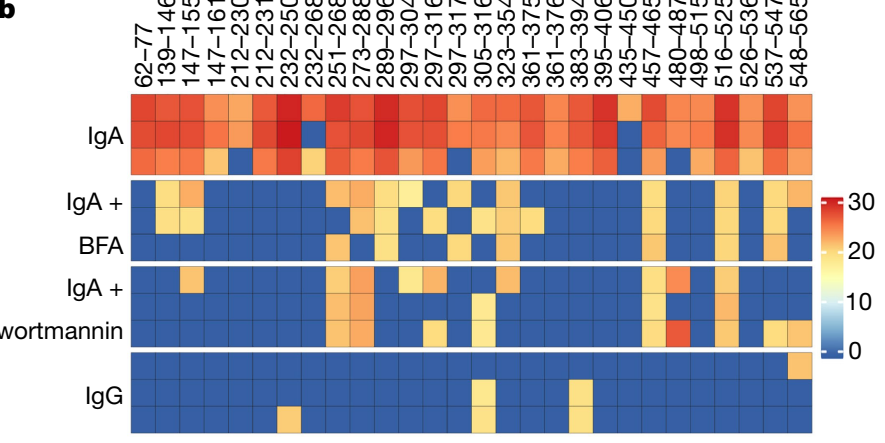

d

GO pathways upregulated by $\lg A$ Inflammatory response

Response to tumour necrosis factor Response to interleukin 1 Response to chemokine

Regulation of inflammatory response Interleukin 6 production

Response to cytokine

Cytokine receptor binding Acute inflammatory response Myelolid leukocyte activation Chemokine receptor binding

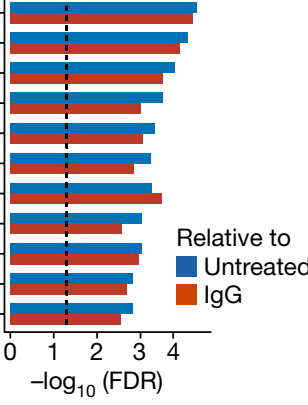

$\mathbf{f}$
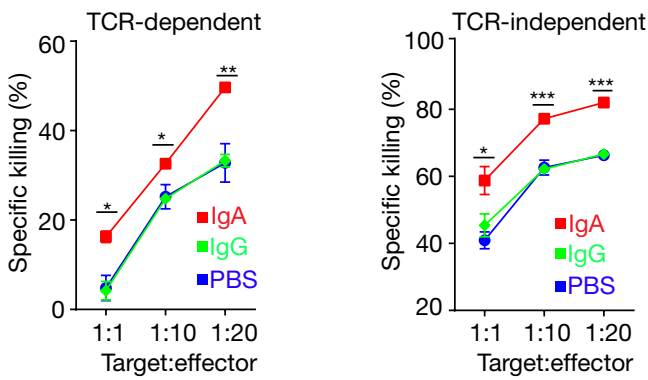

Fig. 2 |See next page for caption.

secretory component were detected in the supernatants of OVCAR3, OVCAR4, OVCAR5 or primary ovarian cancer cells incubated with IgA, but not when these cells were co-incubated with the transcytosis inhibitors wortmannin and brefeldin $\mathrm{A}^{9,10}$, or when cells were incubated with IgG (Fig. 2b, Extended Data Fig. 4b-e, Supplementary Data 1,2). Finally, IgA co-immunoprecipitated with the secretory component in OVCAR3 supernatants, and this was again abolished by transcytosis inhibitors or PIGR ablation in tumour cells (Fig. 2c, Supplementary Data 3).
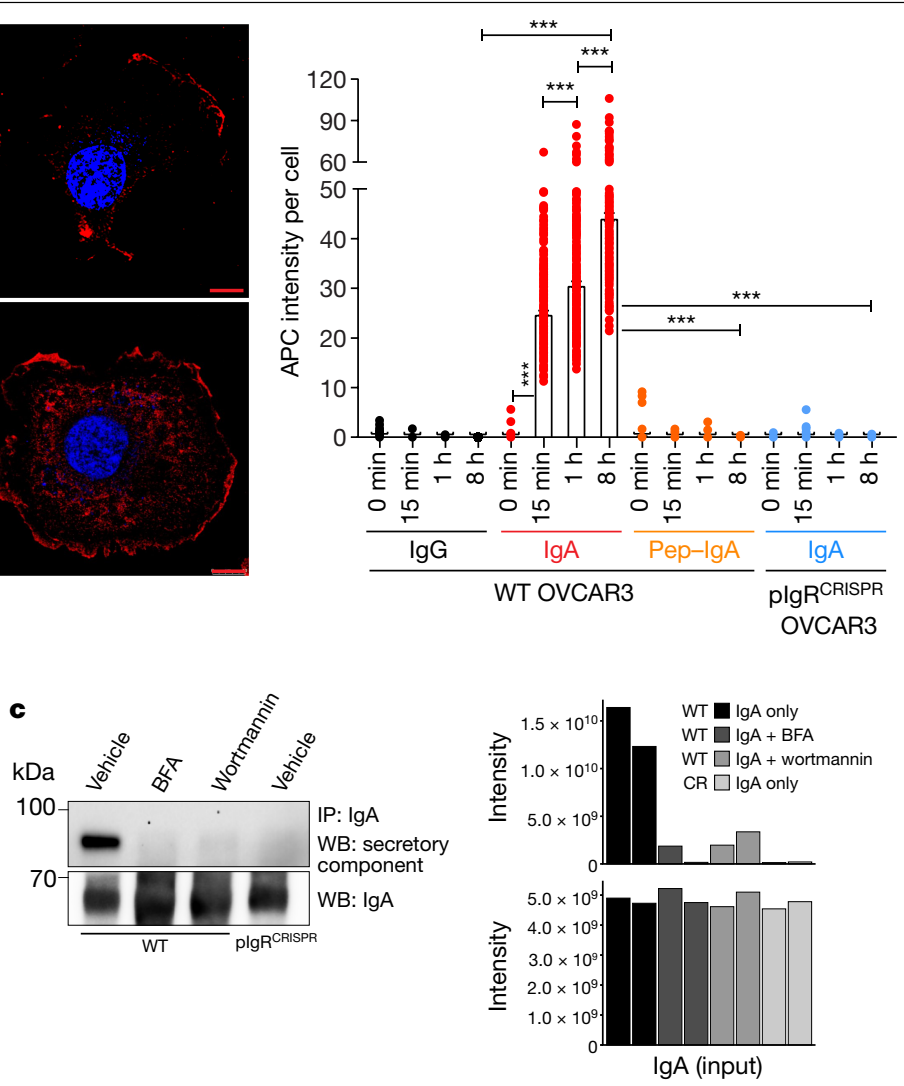

e $\lg A$-treated OVCAR3

IgG-treated OVCAR3
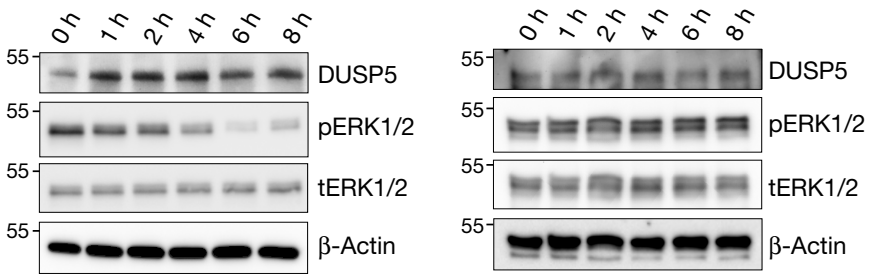

g
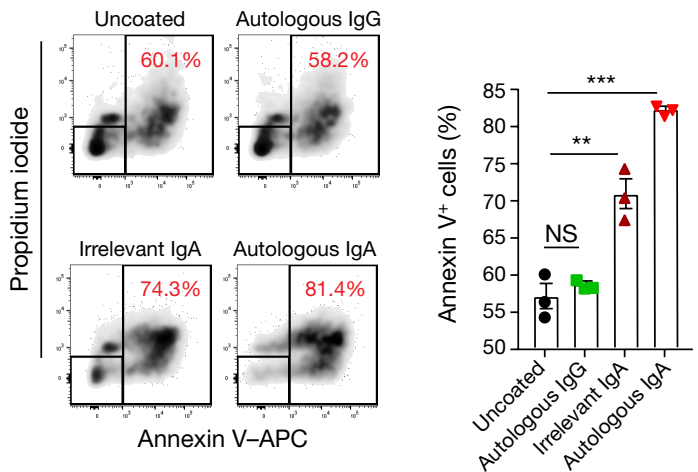

Notably, IgA transcytosis induced broad transcriptional changes in inflammatory pathways in tumour cells, including the upregulation of IFN $\gamma$ receptors (Fig. 2d, Extended Data Fig. 4f, g) and downregulation of tumour-promoting ephrins ${ }^{11}$ (Extended Data Fig. $4 g$, Supplementary Data 4). In addition, several DUSP phosphatases-which are known to counteract phosphorylation events downstream of the RAS pathway ${ }^{12}$-were simultaneously increased upon incubation with non-antigen-specific IgA (but not IgG or vehicle, or in PIGR-ablated, IgA-treated cells), at both the mRNA (Extended Data Fig. 4g) and 
Fig. 2 Transcytosis of IgA through plgR $^{+}$ovarian cancer cells impairs tumour growth and augments cytotoxic killing mediated by $T$ cells. a, Left images of APC-labelled IgA binding and internalization in plgR $^{+}$OVCAR 3 cells (repeated three times). Scale bar, $50 \mu \mathrm{m}$ (main panels), $10 \mu \mathrm{m}$ (magnified regions). Right, comparison of antibody internalization signal (mean \pm s.e.m.) in different treatment conditions and at different temporal points. Each dot represents quantification from one cell. ${ }^{* * *} P \leq 0.001$, unpaired two-tailed $t$-test. Supplementary Table 2 provides details of statistics. b, OVCAR 3 cells were incubated with control IgA or IgG for $8 \mathrm{~h}$ in the presence of wortmannin, brefeldin A (BFA) or vehicle, and supernatants were subjected to liquid chromatography with tandem mass spectrometry (LC-MS/MS). Heat map of all peptides of the extracellular domain of $\operatorname{plgR}(n=3)$; scale represents $\log _{2}$-transformed intensities of plgR peptide fragments detected in LC-MS/ MS.c, Left, co-immunoprecipitates of supernatants from IgA-treated $\mathrm{plgR}^{+}$or PIGR-ablated OVCAR 3 cells (with and without brefeldin A or wortmannin) blotted for the secretory component of plgR and IgA (input control). Right, LC-MS/MS analysis of the co-immunoprecipitates showing intensities ( $\log _{2}$-transformed) of the secretory component of plgR and IgA $(n=2)$. WT, wild type; $\mathrm{CR}, P I G R$-ablated.d, Pre-ranked gene-set enrichment analysis (GSEA), showing the top upregulated gene sets in OVCAR3 cells treated with irrelevant IgA compared to IgG or untreated cells $(n=3)$, Kolmogorov-Smirnov test. GO, Gene Ontology.e, Progressive increase in DUSP5 and concomitant reduction in phospho-ERK1 and phospho-ERK2 (pERK1/2) after IgA treatment (left) of OVCAR3 cells, but not IgG treatment (right). Experiments were repeated three times. tERK1/2, total ERK1 and total ERK2.f, Left, dose-dependent cytotoxic killing of NY-ESO-1-transduced OVCAR3 cells $(n=3)$ by NY-ESO-1TCR-transduced T cells is augmented by co-incubation with IgA, compared to IgG or PBS. Right, IgA treatment also augmented the anti-tumour activity of FSH-targeted chimeric receptor T cells. Mean \pm s.e.m. ${ }^{*} P \leq 0.05,{ }^{* *} P \leq 0.01$, ${ }^{* * *} P \leq 0.001$, ordinary one-way ANOVA. Supplementary Table 2 provides details of statistics. g, Cytotoxic killing of primary $\mathrm{CD} 45^{-} \mathrm{EpCAM}^{+}$tumour cells $(n=3)$ by autologous tumour-infiltrating T cells (1:1 ratio) is augmented by co-incubation with autologous $(P<0.0001)$ or irrelevant $\operatorname{lgA}(P=0.0031)$, but not autologous $\operatorname{IgG}(P=0.1951)$. Mean \pm s.e.m. ${ }^{* *} P \leq 0.01,{ }^{* * *} P \leq 0.001$, NS, not significant; unpaired two-tailed $t$-test. protein levels (Fig. 2e, Extended Data Fig. 4h). Finally, increases in DUSP5 were associated with impaired MEK-ERK signalling, as demonstrated by reduced levels of phospho-ERK1 and phospho-ERK2 (Fig. 2e).

To define the functional relevance of phenotypic changes induced by IgA transcytosis in ovarian cancer cells, we expressed the cancer testis antigen NY-ESO- 1 in HLA-A2 ${ }^{+} \mathrm{FSHR}^{+}$OVCAR3 HGSOC cells, as well as an HLA A2-restricted T cell receptor (TCR) in human T cells that recognizes SLLMWITQC (which corresponds to amino acids 157-165 of NY-ESO-1) ${ }^{13}$. The dose-dependent cytotoxic activity of tumour-antigen-redirected T cells was enhanced upon incubation with irrelevant IgA, compared to control IgG or vehicle (Fig. 2f, left). These effects were independent of changes in MHC-I expression, as the cytotoxic activity of human T cells engineered to express an FSH-targeted chimeric receptor ${ }^{14}$-which recognizes FSHR in OVCAR3 cells independently of MHC-I-was enhanced to a similar extent (Fig. 2f, right). Comparable IgA-dependent sensitization of tumour cells to T-cell-mediated killing was identified using expanded tumour-infiltrating lymphocytes and autologous tumour cells from different patients (Fig. 2g), and a similar enhancement was observed using different tumour antigen-specific IgA (Extended Data Fig. 4i). Increased T cell cytotoxicity required interaction between the Fc domain of IgA and pIgR, because it was abolished using pepsinized antibodies or PIGR-ablated OVCAR3 cells (Extended Data Fig. 4j, k). Accordingly, treatment with non-antigen-specific IgA significantly delayed the growth of OVCAR3 tumours in RAG1-deficient tumour-bearing mice, compared to control IgG or pepsinized IgA (Extended Data Fig. 5a-c).Suppression of tumour growth was not due to any tumour-promoting effect of $\operatorname{IgG}^{15}$, as tumour-bearing mice treated with PBS or pepsin-treated $\left(\mathrm{F}\left(\mathrm{ab}^{\prime}\right)_{2}\right)$ immunoglobulin fragments grew at the same rate as their control IgG-treated counterparts (Extended Data Fig. 5b, c).

To define the relative contribution of antigen recognition by tumour-derived IgA to delayed malignant progression, we produced recombinant dimeric IgA antibodies by cloning the matching $\mathrm{VH}$ and $\mathrm{VL}$ sequences of three clonally expanded, IgA-producing tumour-derived B cells, as determined by single-cell B cell receptor sequencing (Supplementary Data 5). All recombinant antibodies abrogated the progression of autologous cancer cells in vivo more effectively than did control IgA antibodies (Fig. 3a, b).

To identify the specificities of antibodies that are spontaneously generated in ovarian cancer, we optimized a system for the isolation, activation, immortalization and characterization of $B$ cells immunopurified from ten freshly dissociated HGSOCs, using human proteome arrays (Extended Data Fig. 5d). We found that IgA and IgG antibodies secreted by tumour-derived $B$ cells recognized a broad range of tumour antigens, many of which have extracellular domains or represent secreted proteins (Supplementary Data 6). To define the functional relevance of extracellular antigen recognition, we focused on TSPAN7 (a tetraspanin that is overexpressed in human carcinomas $^{16}$ ) and on BDNF (a secreted molecule that is associated with poor prognosis in $\mathrm{HGSOC}^{17}$ ) (Extended Data Fig. 5e). We tetramerized a battery of biotinylated 16-20-mer peptides contained in the extracellular domains of these molecules using fluorescent streptavidin, and sorted tetramer-reactive B cells by fluorescence-activated cell sorting (FACS) from our immortalized batches of intratumoural B cells, and cultured them separately (Extended Data Fig. 5f). These B cells predominately produced IgA (Extended Data Fig. 5g) that specifically recognized these targets expressed in HGSOC tumour cells, as well as recombinant TSPAN7 and BDNF (Fig. 3c). Notably, both TSPAN7- and BDNF-reactive IgA: (1) antagonized tumour growth in vivo more effectively than did irrelevant IgA (Fig. 3d); (2) induced areas of central necrosis and $\mathrm{TUNEL}^{+}$cells (Fig. 3e, Extended Data Fig. $5 \mathrm{~h}-\mathrm{j}$ ); and (3) was engulfed by tumour cells more effectively than was irrelevant IgA (Extended Data Fig. 5k). The anti-tumour effects of BDNF-specific antibodies were retained upon removal of the Fc domain (which suggests the neutralization of secreted BDNF), and pepsinized anti-TSPAN7 antibodies lost their anti-tumour activity, which is suggestive of antibody-dependent cellular cytotoxicity or phagocytosis (Fig. 3f). Accordingly, the superior activity of TSPAN7 antibodies (as compared to control IgA) disappeared in NOD-SCID-gamma (NSG) mice, which lack functional macrophages, dendritic cells and natural killer cells (Fig. 4a). Natural killer cell depletion in tumour-bearing RAG1-knockout mice (Extended Data Fig. 6a) had no effect on anti-tumour activity (Fig. 4b). In further support of antibody-dependent cellular phagocytosis, splenic myeloid cells from tumour-bearing (CD89-deficient) mice bound IgA through Fc $\alpha / \mu R$ (CD351) (Fig. 4c, Extended Data Fig. 6b), and killed OVCAR3 targets more effectively upon coating with TSPAN7-reactive IgA (Fig. 4d). Importantly, there were increases in CD351 ${ }^{+}$myeloid cells at tumour beds after treatment with TSPAN7 antibodies as compared to control IgA (Fig. 4e, Extended Data Fig. 6c). Therefore, polyclonal tumour antigen-specific IgA responses hinder malignant progression through at least two independent mechanisms.

To define the role of plgR-mediated IgA transcytosis in anti-tumour activity, we challenged RAG1-deficient mice with PIGR-ablated OVCAR3 tumours. Notably, the protective effect of non-antigen-specific IgA disappeared in both RAG1-deficient and NSG mice (Fig. 4f, Extended Data Fig. 6d) and tumour-derived TSPAN7 and BDNF antibodies showed decreased anti-tumour effects, consistent with the capacity to transcytose through tumour cells (Extended Data Fig. 6e, f, Supplementary Data 7). In further support of the relevance of antigen recognition, significant anti-tumour effects were elicited by clonally expanded 

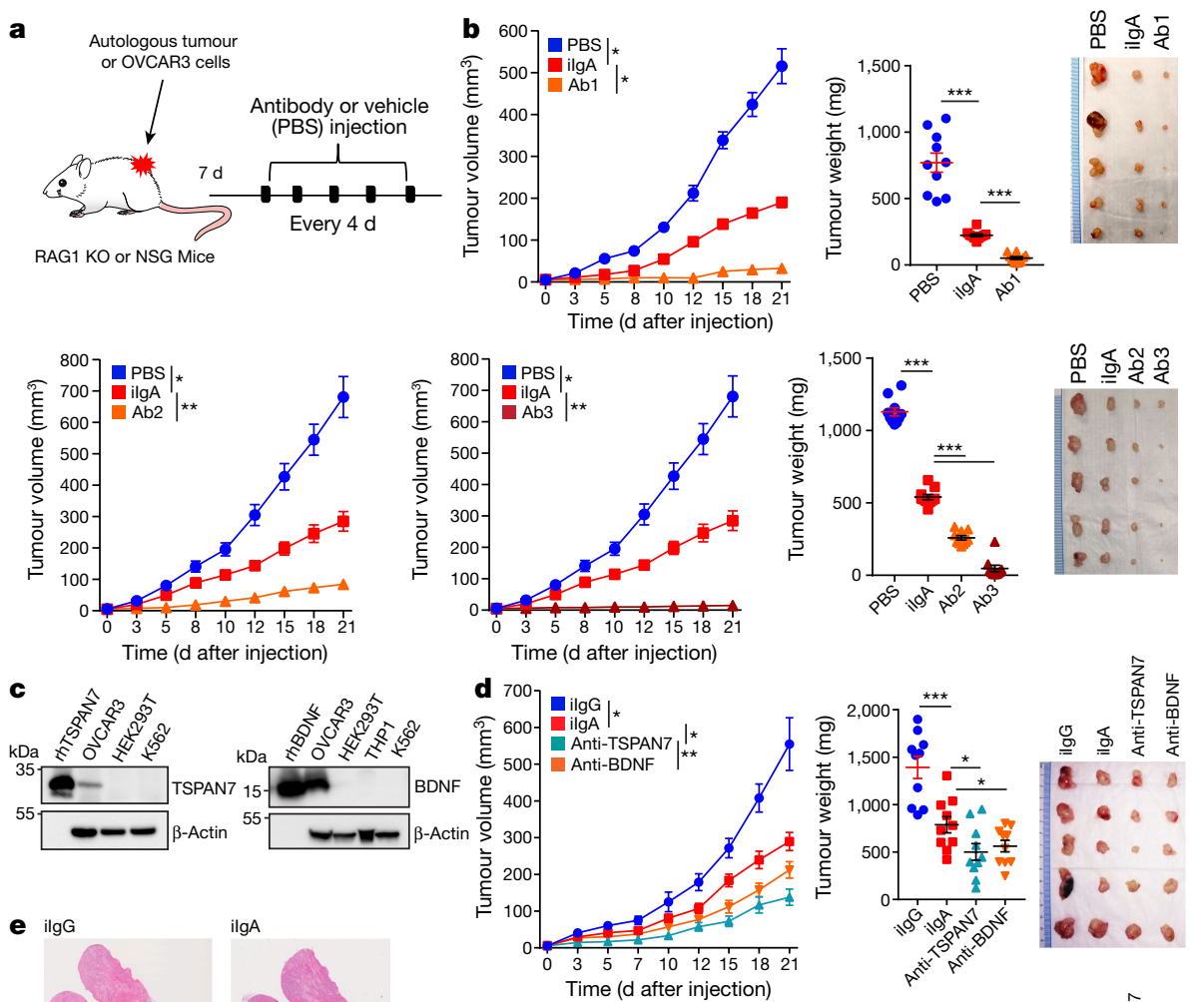

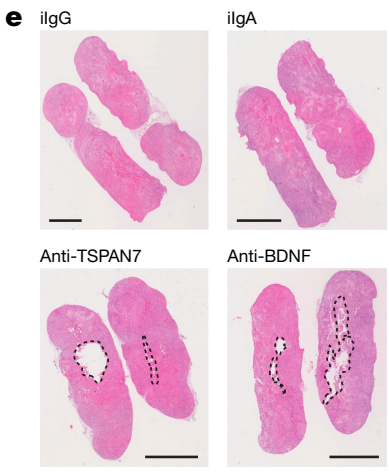

Fig. 3 | Tumour-antigen-specific IgA produced in the ovarian cancer microenvironment antagonizes ovarian cancer progression. a, Schematic of design of experiment shown in b, d,f. Antibody at $100 \mu \mathrm{g}$ per $20 \mathrm{~g}$ body weight or equal volume of vehicle (PBS) was intratumourally injected. $\mathrm{KO}$, knockout.b, Autologous tumour growth curves (left), weight (centre) and volume (right) in tumour-bearing RAG1-knockout mice receiving control (irrelevant IgA (ilgA)) or recombinant dimeric IgA antibodies (labelled Ab1, Ab2 or Ab3) produced with three different matching IgA sequences clonally expanded in two different HGSOC. Respective autologous HGSOC cells were used (tumour no. 1 for Ab1 and tumour no. 2 for Ab2 or Ab3). Supplementary Table 3 provides details of statistics. c, IgA purified from TSPAN7- and BDNF-reactive immortalized B cells recognizes the corresponding recombinant proteins in western blot analysis, along with endogenous TSPAN7 and BDNF expressed in OVCAR3 cells. HEK293T, THP1 and K562 cells were included as negative controls. Experiments were repeated three times.

IgA, but not by control irrelevant IgA against $P I G R$-ablated autologous tumours (Extended Data Figs. 3c, 6g).

Collectively these data demonstrate that IgA produced in the ovarian cancer microenvironment contributes to thwarting malignant progression through both antigen recognition, and via non-specific transcytosis through $\mathrm{plgR}^{+}$tumour cells. These findings indicate that immunotherapies that boost both coordinated $\mathrm{B}$ and $\mathrm{T}$ cell responses against human ovarian cancer-an immunogenic disease that is currently resistant to checkpoint inhibitors-are likely to show increased therapeutic benefit. In support of this notion, a similar synergy has previously been suggested for other malignancies ${ }^{18-21}$. Furthermore,
rhTSPAN7, recombinant human TSPAN7; rhBDNF, recombinant human BDNF. d, Tumour growth curves (left), weight (centre) and tumour volume (right) in tumour-bearing RAG1-knockout mice receiving control or tumour-derived antibodies. Supplementary Table 4 provides details of statistics.e, Representative images ( $n=10$ per group from 2 independent experiments) of central necrosis in tumours from mice receiving IgA from tumour-derived B cells. Scale bars, $4 \mathrm{~mm}$. $\mathrm{f}$, Antibodies used in $\mathbf{d}$ were digested with pepsin to remove their $\mathrm{Fc}$ domain and resulting $\mathrm{F}\left(\mathrm{ab}^{\prime}\right)_{2}$ fragments used to treat OVCAR3 tumour-bearing RAG1-knockout mice under identical conditions. Tumour growth curves (left), weight (centre) and tumour volume (right). Supplementary Table 5 provides details of statistics. In b, d,f, growth curves and tumour weights were pooled from 2 independent experiments ( $n=10$ mice per group in total). Data are mean \pm s.e.m. ${ }^{*} P \leq 0.05,{ }^{* *} P \leq 0.01,{ }^{* * *} P \leq 0.001$, NS, not significant; paired two-tailed $t$-test for growth curves or unpaired two-tailed $t$-test for tumour weights.

IgA-based immunotherapies could be more effective than conventional IgG-targeted interventions against ovarian cancer or other plgR $^{+}$ mucosal tumours.

\section{Online content}

Any methods, additional references, Nature Research reporting summaries, source data, extended data, supplementary information, acknowledgements, peer review information; details of author contributions and competing interests; and statements of data and code availability are available at https://doi.org/10.1038/s41586-020-03144-0. 

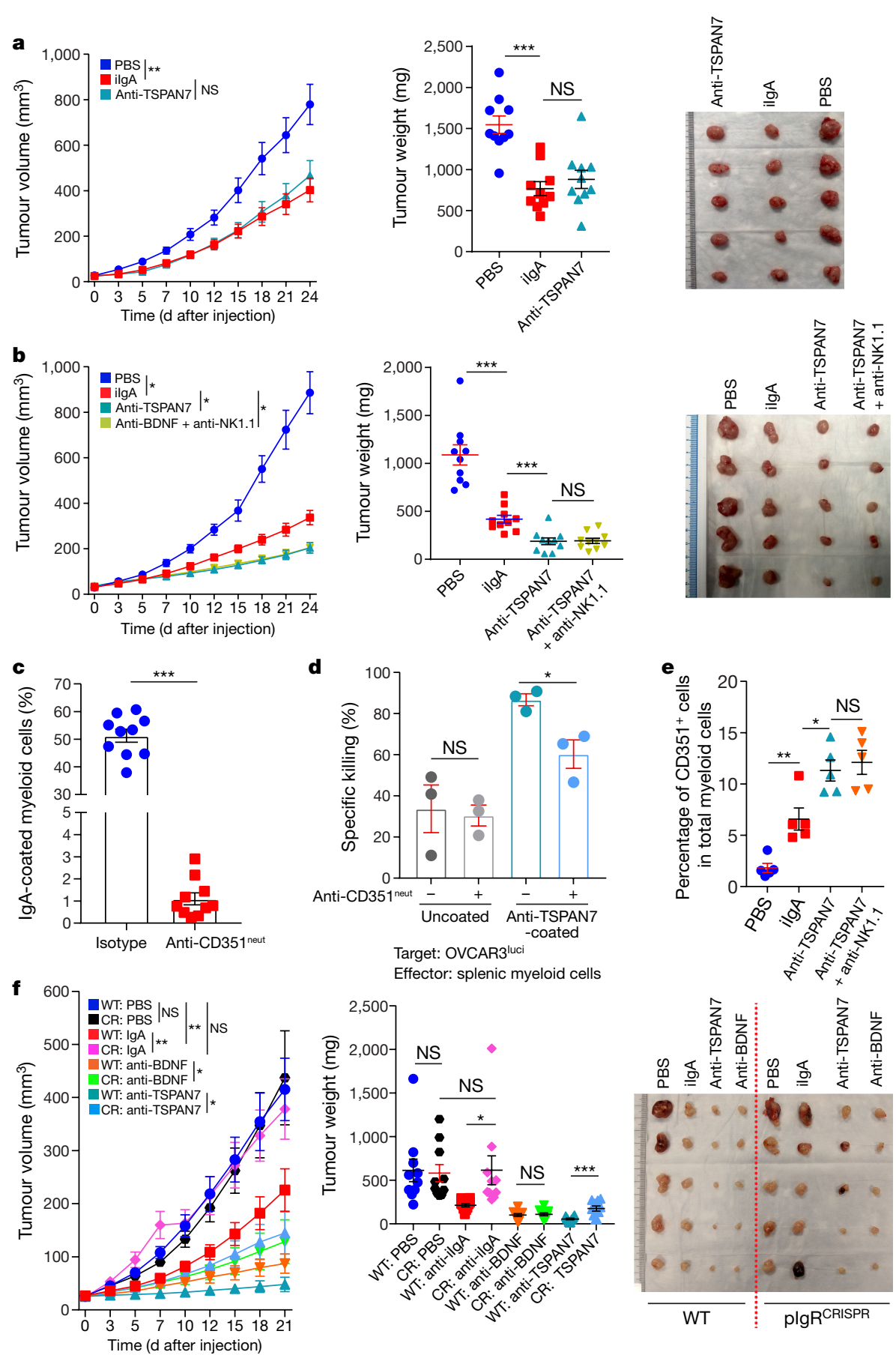

Fig. 4 | Antigen-specific IgA redirects $F c \alpha / \mu R$-positive myeloid cells against cell-surface antigen-positive tumour cells. a, Tumour growth curves (left), tumour weight (centre) and volume (right) in tumour-bearing NSG mice receiving control or tumour-derived antibodies. Supplementary Table 6 provides for details of statistics. b. Tumour growth curves (left), tumour weight (centre) and volume (right) in tumour-bearing RAG1-knockout mice receiving control or tumour-derived antibodies \pm intraperitoneal injections of anti-NK1.1 or control antibodies. Supplementary Table 7 provides for details of statistics. In $\mathbf{a}, \mathbf{b}$, growth curves and tumour weights were pooled from 2 independent experiments ( $n=10$ mice per group in total). c, Binding of IgA antibodies to splenic CD11b ${ }^{+}$cells from tumour-bearing RAG1-knockout mice $(n=10)$, after incubation with $\mathrm{Fc} \alpha / \mu \mathrm{R}$ (CD351)-neutralizing antibodies or isotype controls. $P<0.0001$. d, Cytotoxic killing of OVCAR3 cells by splenic myeloid cells from tumour-bearing RAG1-knockout mice (1:1 ratio) is augmented by coating the tumour cells with anti-TSPAN7, and inhibited by neutralizing CD351
(anti-CD351 $\left.{ }^{\text {neut }}\right)(n=3)$. Supplementary Table 8 provides details of statistics. OVCAR $3^{\text {Luci, }}$, OVCAR3 cells transduced with luciferase-expressing vector. e, Increased accumulation of CD351 $1^{+}$myeloid cells in xenografts in RAG1-knockout mice treated with intratumoural anti-TSPAN7, compared to irrelevant IgA or vehicle, irrespectively of NK1.1 depletion $(n=5)$. Supplementary Table 8 provides for details of statistics.f, Tumour growth curves (left), tumour weight (centre) and volume (right) in wild-type IgR $^{+}$ (WT) or PIGR-ablated (pIgR ${ }^{\text {CRISPR }}$ (CR)) OVCAR3 tumour-bearing RAG1-knockout mice receiving control or tumour-derived antibodies. Two independent experiments were performed with similar results; tumour growth was represented from 1 experiment ( $n=5$ mice per group); tumour weights were pooled from 2 experiments ( $n=10$ mice per group in total). Supplementary Table 9 provides details of statistics. Data are mean \pm s.e.m. ${ }^{*} P \leq 0.05,{ }^{* *} P \leq 0.01$, ${ }^{* * *} P \leq 0.001$, NS, not significant; paired two-tailed $t$-test for growth curves or unpaired two-tailed $t$-test for tumour weights and other experiments. 


\section{Article}

1. Curiel, T. J. et al. Specific recruitment of regulatory T cells in ovarian carcinoma fosters immune privilege and predicts reduced survival. Nat. Med. 10, 942-949 (2004).

2. Zhang, L. et al. Intratumoral T cells, recurrence, and survival in epithelial ovarian cancer. N. Engl. J. Med. 348, 203-213 (2003).

3. Sato, E. et al. Intraepithelial $\mathrm{CD} 8^{+}$tumor-infiltrating lymphocytes and a high $\mathrm{CD} 8^{+}$ regulatory $T$ cell ratio are associated with favorable prognosis in ovarian cancer. Proc. Natl Acad. Sci. USA 102, 18538-18543 (2005).

4. Boland, J. L. et al. Early disease progression and treatment discontinuation in patients with advanced ovarian cancer receiving immune checkpoint blockade. Gynecol. Oncol. 152, 251-258 (2019)

5. Montfort, A. et al. A strong B-cell response is part of the immune landscape in human high-grade serous ovarian metastases. Clin. Cancer Res. 23, 250-262 (2017).

6. Kroeger, D. R., Milne, K. \& Nelson, B. H. Tumor-infiltrating plasma cells are associated with tertiary lymphoid structures, cytolytic T-cell responses, and superior prognosis in ovarian cancer. Clin. Cancer Res. 22, 3005-3015 (2016).

7. Payne, K. K. et al. BTN3A1 governs antitumor responses by coordinating $a \beta$ and $\gamma \delta T$ cells. Science 369, 942-949 (2020).

8. Sautès-Fridman, C., Petitprez, F., Calderaro, J. \& Fridman, W. H. Tertiary lymphoid structures in the era of cancer immunotherapy. Nat. Rev. Cancer 19, 307-325 (2019).

9. Cardone, M. \& Mostov, K. Wortmannin inhibits transcytosis of dimeric IgA by the polymeric immunoglobulin receptor. FEBS Lett. 376, 74-76 (1995).

10. Hunziker, W., Whitney, J. A. \& Mellman, I. Selective inhibition of transcytosis by brefeldin A in MDCK cells. Cell 67, 617-627 (1991).

11. Boyd, A. W., Bartlett, P. F. \& Lackmann, M. Therapeutic targeting of EPH receptors and their ligands. Nat. Rev. Drug Discov. 13, 39-62 (2014).

12. Patterson, K. I., Brummer, T., O'Brien, P. M. \& Daly, R. J. Dual-specificity phosphatases: critical regulators with diverse cellular targets. Biochem. J. 418, 475-489 (2009).

13. Robbins, P. F. et al. Tumor regression in patients with metastatic synovial cell sarcoma and melanoma using genetically engineered lymphocytes reactive with NY-ESO-1. J. Clin. Oncol. 29, 917-924 (2011).
14. Perales-Puchalt, A. et al. Follicle-stimulating hormone receptor is expressed by most ovarian cancer subtypes and is a safe and effective immunotherapeutic target. Clin. Cancer Res. 23, 441-453 (2017).

15. Andreu, P. et al. FcRy activation regulates inflammation-associated squamous carcinogenesis. Cancer Cell 17, 121-134 (2010).

16. Romanska, H. M. \& Berditchevski, F. Tetraspanins in human epithelial malignancies. J. Pathol. 223, 4-14 (2011).

17. Mysona, D. et al. A combined score of clinical factors and serum proteins can predict time to recurrence in high grade serous ovarian cancer. Gynecol. Oncol. 152, 574-580 (2019).

18. Rasoulouniriana, D. et al. A distinct subset of FcyRI-expressing Th1 cells exer antibody-mediated cytotoxic activity. J. Clin. Invest. 129, 4151-4164 (2019).

19. Cabrita, R. et al. Tertiary lymphoid structures improve immunotherapy and survival in melanoma. Nature 577, 561-565 (2020)

20. Helmink, B. A. et al. B cells and tertiary lymphoid structures promote immunotherapy response. Nature 577, 549-555 (2020)

21. Petitprez, F. et al. B cells are associated with survival and immunotherapy response in sarcoma. Nature 577, 556-560 (2020).

Publisher's note Springer Nature remains neutral with regard to jurisdictional claims in published maps and institutional affiliations.

Open Access This article is licensed under a Creative Commons Attribution 4.0 International License, which permits use, sharing, adaptation, distribution and reproduction in any medium or format, as long as you give appropriate credit to the original author(s) and the source, provide a link to the Creative Commons license, and indicate if changes were made. The images or other third party material in this article are included in the article's Creative Commons license, unless indicated otherwise in a credit line to the material. If material is not included in the article's Creative Commons license and your intended use is not permitted by statutory regulation or exceeds the permitted use, you will need to obtain permission directly from the copyright holder. To view a copy of this license, visit http://creativecommons.org/licenses/by/4.0/.

(c) The Author(s) 2021 


\section{Methods}

No statistical methods were used to predetermine sample size. The experiments were not randomized. Investigators were not blinded to allocation during experiments and outcome assessment, unless otherwise mentioned.

\section{Human samples}

Human ovarian carcinoma tissues were procured under protocols approved by the Committee for the Protection of Human Subjects at Dartmouth-Hitchcock Medical Center (no. 17702); and under a protocol approved by the H. Lee Moffitt Cancer Center (MCC no. 19767 and MCC no. 18974). Part of the tumour tissues were either snap-frozen for protein extraction, or were freshly dissociated and cryopreserved. Viable cells in thawed samples were gated for FACS analysis using viability dyes (BioLegend). We have analysed tissue microarrays (TMAs) procured from the Moffitt Cancer Center Tissues Core, which include 93 HGSOC and some control tissues (MCC cohort) (approval MCC no. 50264). Tumour tissue from 261 HGSOCs from 2 prospective cohort studies (the Nurses' Health Study (NHS) and NHSII ${ }^{22}$ ) and from 180 HGSOCs from a population-based case-control study (New England Case-Control Study (NECC cohort)) were used. In brief, the NHS enrolled 121,700 female registered nurses, aged $30-55$, residing in 1 of 11 states of the USA in 1976 . Similarly, the NHSII enrolled 116,429 female registered nurses aged $25-42$ residing in 1 of 14 states of the USA in 1989. At baseline, women completed a mailed baseline questionnaire on their lifestyle and medical history. Questionnaires to collect updated information have since been mailed biennially. Incident epithelial ovarian cancer cases were identified from questionnaires, reports from family or linkage to the National Death Index, and confirmed by medical record review or cancer registry linkage. The study protocol was approved by the institutional review boards of the Brigham and Women's Hospital and Harvard T. H. Chan School of Public Health, and those of participating registries as required. Return of the questionnaires is considered to imply informed consent.

The NECC used state-wide cancer registries and hospital tumour boards to identify 2,040 cases of epithelial ovarian cancer from eastern Massachusetts and New Hampshire from 1992 to $2008^{23}$. Women were ineligible if they were younger than age 18 , did not have a telephone, did not speak English, moved, died, had a prior bilateral oophorectomy or their physician declined permission to contact. The institutional review boards at Brigham and Women's Hospital and Dartmouth Medical School approved the study protocol, and each participant provided written informed consent.

The NHS and NHSII requested paraffin-embedded tissue blocks containing representative tumour samples from cases of epithelial ovarian cancer with a pathology report. The NECC accessed tumour blocks from patients, most of whom were diagnosed at Brigham and Women's Hospital. In NECC, funding was available to obtain tissue blocks for only a subset of cases, oversampling high-grade serous tumours. Tissue blocks were reviewed to verify histology and grade and make TMAs. TMAs were arrayed at the Dana-Farber/Harvard Cancer Center Specialized Histopathology Core by taking at least three, and up to six, core biopsies with a $1.0 \mathrm{~mm}$ (NECC) or $0.6 \mathrm{~mm}$ (NECC, NHS and NHSII) diameter from ovarian cancer tissue blocks and re-embedding the cores into a single block. All specimens of HGSOC used and analysed in this study are described in Supplementary Table 10.

\section{Cell lines}

OVCAR3, A549, NIH-H23, HEK-293T, K562 and THP1 cells were purchased from ATCC; OVCAR4, OVCAR5 and OVACR8 cells were procured from National Cancer Institute; Kuramochi cell line was procured fromJCRB Cell Bank. Human OSE cells were purchased from ScienCell Research Laboratories (no. 7310). We transduced OVCAR3 tumour cells with a luciferase-expressing vector (OVCAR $3^{\text {luci }}$ ). To make OVCAR3 cells as target of NY-ESO-1-specific T cells, OVCAR3 ${ }^{\text {luci }}$ cells were transduced to express NY-ESO-1(OVCAR3 ${ }^{\text {luci }}{ }^{-N Y-E S O-1)}$. Tumour-sorted CD45-EpCAM ${ }^{+}$ primary HGSOC cells were cultured continuously in R10 medium (RPMI-1640, 10\% FBS, penicillin $\left(100 \mathrm{IU} \mathrm{ml}^{-1}\right)$, streptomycin $(100 \mu \mathrm{g}$ $\left.\mathrm{ml}^{-1}\right)$, L-glutamine $(2 \mathrm{mM})$, sodium pyruvate $(0.5 \mathrm{mM})$ ) (ThermoScientific) until they adhere and grow similar to a cell line. All cell lines, except OSE, were routinely cultured in R10 medium. OSE cells were routinely cultured in recommended complete medium, purchased from ScienCell Research Laboratories. Cell lines routinely tested for negative mycoplasma contamination.

\section{CRISPR-Cas9-mediated ablation of PIGR in OVCAR3 cells}

CRISPR RNA (crRNA) targeting PIGR 5'-CUUCACAACAGAGCGA CGAUGUUUUAGAGCUAGAAA-3' (IDT) was reconstituted to make $100 \mu \mathrm{M}$ in nuclease-free duplex buffer (IDT), and then mixed at equimolar concentration with Alt-R CRISPR-Cas9 trans-acting crRNA (tracrRNA), Atto550 (IDT) in a sterile PCR tube. Guide RNA was prepared by annealing crRNA and tracrRNA duplexes by heating at $95^{\circ} \mathrm{C}$ for 5 min in PCR thermocycler, followed by gradual slow cooling to room temperature. Nine $\mu \mathrm{l}$ of crRNA-tracrRNA duplexes was mixed with $6 \mu \mathrm{l}(180 \mathrm{pmol})$ of TrueCut Cas9 Protein v.2 (Invitrogen), followed by incubation at room temperature for 10 min to form Cas9 ribonucleoproteins (RNPs). OVACR3 or primary human HGSOC cells were suspended into final concentration of $5 \times 10^{6}$ cells per ml. Fifteen microlitres of the Cas 9 RNPs was added to $100 \mu \mathrm{l}$ of the cell suspension and electroporated using Neon Tranfection System (ThermoFisher), performed at $1170 \mathrm{~V}$, $30 \mathrm{~ms}$ for 2 pulses. Cells were then cultured in R10 medium. Electroporation was confirmed by analysing Atto500 (tracrRNA) signal by flow cytometry on the next day, and loss of pIgR from the OVACR3 cells or primary HGSOC cells was confirmed by western blot five days later.

\section{Mice and tumours}

Female, 4-6-week-old Rag1-deficient $\left(\operatorname{Rag} 1^{-/-}\right)$mice and NSG mice of the same age group were procured from Charles River Laboratories and Jackson Laboratory, respectively; and maintained by the animal facility of $\mathrm{H}$. Lee Moffitt Cancer Center, with a 12 light/12 dark cycle, at about $18-23^{\circ} \mathrm{C}$ with $40-60 \%$ humidity. Mouse experiments were approved by the Institutional Animal Care and Use Committee at the University of South Florida.

Flank tumours were initiated by injecting $1 \times 10^{7}$ OVCAR 3 or autologous primary human HGSOC cells (wild-type or PIGR-ablated) cells. Tumour volume was calculated as: $0.5 \times\left(L \times W^{2}\right)$, in which $L$ is length and $W$ is width. Tumour tissues were dissected mechanically into single-cell suspensions for flow cytometry, or retained for RNA and protein isolation.

Intratumoural or peritumoural injections of antibodies were done on several days, starting from day 7 after the tumour challenge, at a dose of $100 \mu \mathrm{g}$ per $20 \mathrm{~g}$ body weight.

Natural killer cells were depleted from RAG1-deficient mice by intraperitoneal injections of $200 \mu \mathrm{g}$ of NK1.1-neutralizing antibodies (anti-NK1.1, BioXCell, PK136, BE0036) 3 days before tumour challenge, followed by $100-\mu \mathrm{g}$ injections on every 3 or 4 days.

Tumour volumes in mice were measured using code names on the cages and ear tags, instead of specific information about the treatments that the mice received. Apart from this, no blinding method was used for mouse studies.

\section{Ovarian-cancer-sorted B cell immortalization, antibody} purification, proteome array and pepsin digestion

Cryopreserved single-cell suspensions of HGSOC were thawed in $37^{\circ} \mathrm{C}$ water bath followed by clearing of the cryoprotectant medium by centrifugation. After performing annexin $\mathrm{V}^{+}$dead cell removal, viable single-cell samples were bead-sorted with human CD19 microbeads (Miltenyi Biotec, 130-050-301), according to manufacturer's recommendation. Isolated $\mathrm{CD} 19^{+} \mathrm{B}$ cells were counted and $2 \times 10^{5} \mathrm{~B}$ cells per 
ml R10 were expanded for 5 days using human B cell expansion reagents (R\&D Systems, CDK005), according to manufacturer's recommendation. Expanded B cells were then challenged with Epstein-Barr virus (ATCC-VR-1492) added at 1:10 ratio, and kept in R10 without expansion supplements. In 2 to 3 weeks, most of the Epstein-Barr-virus-infected B cells had died; very few sustained the challenge and become immortalized. Immortalized B cells were grown to confluency. The conditioned medium was collected and concentrated using filters (Millipore Amicon, UFC900325). Part of the concentrated supernatants was analysed for reactivity against human protein antigens, using a proteome array that includes about $81 \%$ of all human proteins spotted into glass slides (CDi Lab).

Specific antigen-reactive B cells were FACS-sorted using tetramers, prepared by biotinylated peptides (GenScript) and PE-labelled streptavidin (BioLegend, 405203), and grown. Peptides were added from a $5.0 \mathrm{mg} \mathrm{ml}^{-1}$ stock to $B$ cells in suspension at a final concentration of $1.0 \mu \mathrm{g}$ per $10^{7} \mathrm{~B}$ cells in $200 \mu \mathrm{l}$ medium and incubated for $30 \mathrm{~min}$ at $4{ }^{\circ} \mathrm{C}$, followed by a wash with PBS and incubation with streptavidin-PE added at 1:20 dilution. We used the peptides HGIPPSCCMNETDCNP and TQSYVRALTMDSKKRI to sort TSPAN7- and BDNF-specific B cells, respectively, from the pool of immortalized $B$ cells.

Sorted cells were diluted in fresh medium and distributed in round-bottom 96-well plates in a way such that each well received one cell. Additional $10,000 \mathrm{~B}$ cells, irradiated with $100 \mathrm{~Gy} \mathrm{X}$-ray and $30 \mathrm{~min}$ $\mathrm{UV}$, were added to each well as feeder cells. Then individual clones of B cells were grown over a period of 1 month. Conditioned medium was collected, concentrated using Amicon filters, and from the concentrated medium, human IgA or IgG were purified using purification kits (LigaTrap, LT-146KIT and LT-095KIT) according to recommended protocols.

To prepare $\mathrm{F}\left(\mathrm{ab}^{\prime}\right)_{2}$ fragments, Fc regions of human IgA or IgG antibodies were pepsin-digested using a pepsin digestion kit (ThermoScientific, 44988) according to the manufacturer's recommendation.

\section{Cytotoxicity assay}

CD3 T cells were bead-sorted from peripheral blood lymphocytes and transduced with a retroviral construct of NY-ESO-1 TCR or human FSH-CER ${ }^{14}$. NY-ESO-1 TCR was made using a sequence corresponding to an HLA-A2-restricted TCR that recognizes SLLMWITQC, corresponding to residues 157 to 165 of NY-ESO-13 (publicly available http:// www.google.com/patents/US8143376), was purchased from IDT and ligated into pBMN-I-GFP. In luciferase-compatible 96-well plates, 10,000 OVCAR3 ${ }^{\text {luci-NY-ESO-1 }}$ or OVCAR $3^{\text {luci }}$ cells were placed. After $4 \mathrm{~h}$ wells were washed, fresh medium was added and cells were treated with non-specific, native human IgA (Abcam, ab91025) or IgG (Abcam, ab98981) at $0.5 \mu \mathrm{g} \mathrm{ml}^{-1}$ final concentration. After $2 \mathrm{~h}$ incubation at $37^{\circ} \mathrm{C}$, we added the appropriate number of $\mathrm{T}$ cells per well in a final volume of $200 \mu \mathrm{l}$. Cells were incubated for $4 \mathrm{~h}$ or $16 \mathrm{~h}$ in a $37-{ }^{\circ} \mathrm{C}$ incubator, and checked for cytotoxicity using the luciferase assay (Promega).

For the autologous cytotoxicity assay, total $\mathrm{CD} 3^{+} \mathrm{T}$ cells, $\mathrm{CD} 19^{+}$ $B$ cells and $\mathrm{CD}^{-} 5^{-} \mathrm{EpCAM}^{+}$tumour epithelial cells were sorted after removal of dead cells. The B cells were immortalized, and IgA or IgG antibodies from concentrated supernatants of growing B cell culture-conditioned medium were separated as described in 'Ovarian-cancer-sorted B cell immortalization, antibody purification, proteome array and pepsin digestion'. The tumour cells were grown in R10 medium to make continuous cultures. Ten thousand tumour cells were placed in 96-well plates. After $4 \mathrm{~h}$ wells were washed, fresh medium was added and cells were treated with autologous B-cell-derived whole or pepsinized IgA, autologous IgG, or irrelevant whole or pepsinized human IgA at $0.5 \mu \mathrm{g} \mathrm{ml}^{-1}$ final concentration. After $2 \mathrm{~h}$ incubation at $37^{\circ} \mathrm{C}$, we added $10,000 \mathrm{~T}$ cells per well in a final volume of $200 \mu \mathrm{l}$. Cells were incubated for $16 \mathrm{~h}$ in a $37-{ }^{\circ} \mathrm{C}$ incubator. Total apoptotic cells (annexin $\mathrm{V}^{+}$) and viable cells (annexin $\mathrm{V}^{-}$propidium iodide $^{-}$) were analysed by flow cytometry.
Wild-type or PIGR-ablated OVACR3 cells were incubated with irrelevant IgA or IgG for $2 \mathrm{~h}, \mathrm{FSH}-\mathrm{CER}$ T cells were added (1:1) and after $16 \mathrm{~h}$ incubation in a $37-{ }^{\circ} \mathrm{C}$ incubator, total apoptotic cells (annexin $\mathrm{V}^{+}$) and viable cells (annexin $\mathrm{V}^{-}$propidium iodide ${ }^{-}$) were analysed by flow cytometry.

Ten thousand OVCAR3 $3^{\text {luci }}$ cells were placed in 96-well plates and after $4 \mathrm{~h}$ wells were washed followed by incubation with or without anti-TSPAN7-IgA $\left(0.5 \mu \mathrm{g} \mathrm{ml}^{-1}\right)$ antibodies for $2 \mathrm{~h}$ at $37^{\circ} \mathrm{C}$, and then splenic myeloid cells from tumour-bearing mice, pre-incubated with isotype or anti-CD351 blocking antibody for $30 \mathrm{~min}$ in ice, were added to the OVACR 3 cells at $1: 1$ ratio and incubated for $12 \mathrm{~h}$, and checked for cytotoxicity using the luciferase assay (Promega).

Cytotoxicity was calculated as (maximum viability control - individual well)/(maximum viability control - maximum death control $) \times 100$, as a percentage.

\section{Multiplex immunohistochemistry}

FFPE TMAs were immunostained using the PerkinElmer OPAL 7-Colour Automation IHC kit on the BOND RX autostainer (Leica Biosystems) and the following anti-human antibodies: CD3 (Dako, A0452, 1:100), CD4 (Cell Marque, EP204, 104R-25, 1:100), CD8 (Dako, C8/144B, M7103,1:800), CD19 (Dako, LE-CD19, M7296, 1:50), CD20 (Dako, L26, M0755, 1:800), CD138 (Dako, MI15, M7228, 1:500), pIgR (Abcam, ab96196, 1:100), IgA (Abcam, EPR5367-76, ab124716, 1:1000), IgG (Abcam, EPR4421, ab109489, 1:500), and pan-cytokeratin (PCK, Dako, AE1/AE3, M3515, 1:200). Nuclei were stained with DAPI. Precisely, tissues were baked at $65^{\circ} \mathrm{C}$ for $2 \mathrm{~h}$ then transferred to the BOND RX (Leica Biosystems) followed by automated deparaffinization, and antigen retrieval using OPAL IHC procedure (PerkinElmer). Autofluorescence slides (negative control) were included, which use primary and secondary antibodies omitting the OPAL fluorophores. Slides were scanned and imaged with the PerkinElmer Vectra3 Automated Quantitative Pathology Imaging System. Multilayer TIFF images were exported from inForm v.2.4.8 (PerkinElmer) and loaded into HALO v.3.0.311.328 (Indica Labs) for quantitative image analysis. Each fluorescent fluorophore is assigned to a dye colour and positivity thresholds were determined per marker on the basis of published nuclear or cytoplasmic staining patterns. Quantifications in tumour islets and stroma were distinguished by PCK staining. Datasets were exported with cytoplasmic, nuclear and total cell counts for each fluorescent marker from the sample set. Cell segmentation files were generated through inForm and dot plots were generated and analysed by FCS Image v.7.0. Standardization of multiplex immunohistochemistry staining experiments with appropriate positive and negative control tissues, including isotype control antibodies, are summarized in Extended Data Figs. 7, 8. Precisely, human tonsil sections were used as a positive control for CD3, CD4, CD8, CD19, CD20, CD138, IgA, IgG and PCK, and as a negative control for plgR. Healthy kidney tissue sections were used as a positive control for pIgR. Glioblastoma sections were used as a negative control for CD3, CD4, CD8, CD19, CD20, CD138, IgA, IgG and PCK. Respective positive-control tissue adjacent sections were stained with isotype control antibodies to rule out false-positive staining.

\section{Microscopy}

Antibody internalization. Whole or pepsinized, non-antigen-specific or specific, IgA or IgG antibodies were conjugated with APC-conjugation kit (Abcam). Fifty thousand OVACR3 cells (wild type or PIGR-ablated) were placed onto a coverslip within 6-well plates and after $12 \mathrm{~h}$ treated with the antibodies. After different hours of incubations, cells were washed, fixed and mounted using a DAPI-containing mounting reagent (CST). Images were captured in a confocal microscope (Leica SP8) using LAS X (v.3.5.5.19976) software. Quantitative acquisition was performed using Zeiss Imager Z2 upright microscope using ZEN 2.3 (blue edition) software. CZI image files were imported into Definiens Tissue Studio v.4.7 (Definiens) to quantify cellular CY5 intensity. The 
software was used to run nucleus and cell detection algorithms to segment each cell, nucleus and cytoplasm and calculate the mean CY5 intensity within in these compartments. Intensity and size thresholds were set to minimize false-positive detection caused by artefacts and background fluorescence.

TUNEL assay. Xenograft tumour FFPE sections were stained for TUNEL ${ }^{+}$ cells using TUNEL Alexa Fluor 647 Imaging assay kit (Thermo) according to the manufacturer's recommendation. Tile images were captured with an ORCA-Flash 4.00 V3 CMOS camera (Hamamatsu Photonics K.K.) and Zen 2 Blue software (Carl Zeiss) and stored in CZI file format. The software was also used to stitch the image tiles into whole-slide images. Representative images were exported to 8-bit TIFF format. CZI image files were imported into Definiens Tissue Studio v.4.7 to quantify the number of TUNEL ${ }^{+}$cells. The software was used to run a nucleus detection algorithm on the CY5 channel of the whole-slide image. Intensity and size thresholds were set to minimize false-positive detection caused artefacts and background fluorescence. An adjacent section for each tumour was stained with haematoxylin and eosin, scanned using automated slide scanner (Aperio-Leica Scanner Console (v.102.0.7.5)) and the images was examined for necrotic holes within the tissues. Each tissue section was manually annotated to determine the area of the entire tissue section and large necrotic holes. The number of TUNEL ${ }^{+}$ cells was normalized by total tissue area minus large necrotic hole areas that appear in many of the tumour sections.

Antibody uptake analysis in xenograft tumours. Xenograft-tumour FFPE sections were rehydrated, antigen was retrieved, blocked and incubated with anti-human IgA antibodies overnight, followed by incubation with Alexa Fluor 647-conjugated secondary antibodies (CST, 4414) and mounted. CZI image files were imported into Definiens Tissue Studio v.4.7 to quantify the number of positive foci in the tissue. The software was used to run a nucleus detection algorithm on the DAPI channel and a spot detection algorithm on the CY5 channel. Intensity and size thresholds were set to minimize false-positive detection caused by artefacts and background fluorescence. The number of positive foci per tissue was normalized by total number of nuclei.

\section{Flow cytometry}

Flow cytometry was performed by staining with Zombie NIR (BioLegend) or Zombie Yellow (BioLegend) or DAPI (ThermoScientific) viability dye, blocking with anti-CD16/32 (BioLegend), and staining for 30 min at $4{ }^{\circ} \mathrm{C}$ with the following anti-human antibodies: CD45 (BD Biosciences, HI30,1:300), CD3 (BD Biosciences, SK7,1:200), CD19 (BD Biosciences, HIB19, 1:200), CD20 (BioLegend, 2H7, 1:200), CD38 (BD Biosciences, HIT2, 1:200), CD138 (BioLegend, MI15, 1:200), CD27 (BD Biosciences, M-T271, 1:200), IgA (Tonbo Biosciences, 35-8016-M001, 1:20), IgG (BioLegend, M1310G05, 1:200), IgM (BioLegend, MHM-88, 1:200), IgD (BD Biosciences, IA6-2, 1:100), IgE (BD Biosciences, G7-26, 1:100), ЕpCAM (BD Biosciences, KS1/4, 1:200), plgR (ThermoScientific, PA5-35340, 1:50) or tetramers against TSPAN7 or BDNF. For intracellular staining for immunoglobulin isotypes, cells were first incubated with surface staining antibodies ( $30 \mathrm{~min}$ in ice), followed by fixation ( $30 \mathrm{~min}$ at room temperature) (eBioscience) and finally incubation with the antibodies for intracellular markers in the permeabilization buffer (eBioscience) (45 min at room temperature).

Mouse xenograft-tumour single-cell suspensions or splenocytes were blocked with Fc blocker (BioLegend) and analysed by flow cytometry after incubation for 30 min at $4{ }^{\circ} \mathrm{C}$ with following anti-mouse antibodies: CD45 (BioLegend, 30-F11), CD11b (BioLegend, M1/70), CD351 (BioLegend, TX61) or with APC-conjugated human IgA. Splenocytes from RAG1-deficient mice were mechanically dissociated and red blood cells were removed, followed by neutralization of $F c \alpha / \mu$ receptor $(\mathrm{Fc} \alpha / \mu \mathrm{R})$ by incubation with CD351-neutralizing antibodies (BioLegend, TX61,137303) or with isotype controls (BioLegend, 400123) at a concentration of $2.0 \mu \mathrm{g}$ per $10^{6}$ cells in $100 \mu$ l volume for $30 \mathrm{~min}$ in ice. After washing, splenocytes were then incubated with APC-conjugated human IgA for another $30 \mathrm{~min}$ in ice and analysed by flow cytometry. Appropriate isotype controls and fluorescence minus one were run. Samples were subsequently run using BD FACS LSRII or sorted using BDFACS ARIA. Data were collected using BD FACS Diva v.8.0.1 and analysed using FlowJo v.10.7.1. Gating strategies used for flow cytometry analyses are summarized in Extended Data Figs. 9, 10.

\section{RNA sequencing}

OVCAR3 cells in culture in 2\% FBS-containing RPMI medium were treated with or without $0.5 \mu \mathrm{g} \mathrm{ml}^{-1}$ of natural human IgA or IgG for $24 \mathrm{~h}$. Total RNA was isolated from cultured cells using RNA isolation kit (Qiagen) and analysed for RINe. An Illumina NextSeq 550 instrument was used to generate 75-basepair paired-end RNA-sequencing (RNA-seq) reads. Base calls were converted to FASTQ files using bcf2 fastq (v.2.20). Paired-end RNA-seq reads were aligned to the GRCh37 human reference genome using $\operatorname{STAR}^{24}(\mathrm{v}$.2.5.3a) following adaptor trimming by cutadapt ${ }^{25}$ (v.1.8.1). For OVCAR3 cells, uniquely mapped reads were counted by feautreCounts ${ }^{26}$ (v.1.5.3) using Gencode V30 transcript annotations for human. Differential expression analysis was performed using DESeq2 (v.1.30.0 $)^{27}$. Heat maps were generated using $z$-score-transformed $\log _{2}(1+$ normalized count $)$.

For each differential expression analysis comparing antibody treated groups with the untreated group, genes were ranked based on $-\log _{10}(P$ value $) \times\left(\right.$ sign of $\log _{2}($ fold change $\left.)\right)$. The preranked gene list was used to perform preranked GSEA ${ }^{28}$ (v.4.0.2) to assess enrichment of hallmarks, curated gene sets and Gene Ontology ${ }^{29}$ terms in $\mathrm{MSigDB}^{28}$. The resulting normalized enrichment score and false-discovery-rate-controlled $P$ values were used to assess the IgA-induced transcriptome changes.

\section{IgA transcytosis and pIgR LC-MS/MS}

One hundred thousand OVCAR3, OVCAR4, OVCAR5 or primary HGSOC-tumour cells were placed in 6-well plates and washed with PBS after $12 \mathrm{~h}$ before treating with or without brefeldin $\mathrm{A}\left(1 \mu \mathrm{g} \mathrm{ml}^{-1}\right)$ or wortmannin $(1 \mu \mathrm{M})$ in serum-free RPMI medium. After $4 \mathrm{~h}$ cells were treated with or without native human IgA (Abcam, ab91025) or IgG (Abcam, ab98981) at $0.5 \mu \mathrm{g} \mathrm{ml}^{-1}$ final concentration. After $12 \mathrm{~h}$, conditioned medium was collected and filtered for contaminant debris removal. Proteins were extracted from the conditioned medium, reduced by DTT, digested by trypsin and subjected to LC-MS/MS analysis by the Moffitt Cancer Center Proteomics Facility. MaxQuant (v.1.5.2.8) was used to analyse the data, identify and quantify the proteins ${ }^{30}$.

\section{Western blot and co-immunoprecipation}

Cells and mechanically dissociated tumour samples were lysed in RIPA buffer (ThermoScientific) with protease-phosphatase inhibitor cocktail (SigmaAldrich) and cleared by centrifugation. Proteins were quantified by BCA assay (ThermoScientific). Membranes were blotted with anti-pIgR (Abcam, ab96196, 1:500), anti-IgA (Abcam, EPR5367-76, ab124716, 1:2,500), anti-phospho-ERK1/2 (CST, D1H6G, 5726, 1:1,000), anti-ERK1/2 (CST, L34F12, 4696, 1:2,000), anti-DUSP5 (Abcam, EPR19684, ab200708, 1:1,000) and anti- $\beta$-actin (CST, 13E5, 5125 or 4970, 1:5,000) antibodies. For TSPAN7 and BDNF, isolated IgA antibodies from concentrated conditioned medium from TSPAN7-and BDNF-reactive B cells were used. Immunoreactive bands were developed using horse radish peroxidase-conjugated secondary antibodies (CST, 7074, 1:5,000; CST, 7076, 1:5,000; Abcam, ab97215, 1:5,000) and enhanced chemiluminescence substrate (GE HealthCare).

HGSOC tumour tissue chunks were pulverized and lysed using nonreducing nondenaturing lysis reagent provided in the co-immunoprecipitation kit (Pierce) used. Pellets of OVCAR3 cells, $\mathrm{CD} 45^{+}$and $\mathrm{CD} 45^{-}$fractions of human ovarian cancer ascites were also lysed with the same nondenaturing lysis reagent. Cell-free human 


\section{Article}

ovarian cancer ascitic fluids were filtered, concentrated and diluted with the nondenaturing lysis reagent. Proteins were immunoprecipitated using anti-human IgA antibody (Abcam, EPR5367-76, ab124716, 1:20) and eluted following the manufacturer's instructions. Elutes and inputs were immunoblotted for plgR and IgA. Conditioned medium from transcytosis experiment in OVCAR3 (wild-type or PIGR-ablated) cells was concentrated and immunoprecipitated with anti-human IgA antibody and part of elutes were western-blotted for the secretory component of pIgR (Abcam, SC-05, ab3924, 1:500) or analysed by LC-MS/MS.

\section{Analysis of TCGA data}

Molecular and clinical data from TCGA for ovarian serous cystadenocarcinoma (designated OV) were downloaded from the cBio Cancer Genomics Portal (http://www.cbioportal.org/), Broad Firehose website (https://gdac.broadinstitute.org/) and Genomic Data Commons Data Portal (https://portal.gdc.cancer.gov/). A total of 428 patients with matched clinical information and tumour RNA-seq data was used in this study. Raw RNA-seq reads were aligned to the GRCh37 human transcriptome using $\mathrm{STAR}^{24}$ (v.2.5.3a). Uniquely aligned reads were counted against Gencode v.19 using htseq-count ${ }^{31}$ (v.0.6.1) and then normalized using DESeq $2{ }^{27}$ taking into account batches and RNA composition bias. All statistical analysis and visualization was performed using $\log _{2}$-transformed normalized count. Kaplan-Meier survival analysis and the log-rank tests were performed to compare overall survival between groups.

\section{X Genomics single-cell V(D)J (BCR) sequencing and recombinant antibody production}

Single-cell V(D)J (BCR) sequencing was performed using the 10XGenomics Chromium system. A single-cell suspension derived from HGSOC-sorted B cells was analysed for viability using the Nexcelom Cellometer K2 and then loaded onto the $10 \mathrm{X}$ Genomics Chromium Single Cell Controller at a concentration of 1,000 cells per $\mu$ l to encapsulate around 5,000 cells per sample. In brief, the single cells, reagents and $10 \mathrm{X}$ Genomics gel beads were encapsulated into individual nanolitre-sized gel beads in emulsion and then reverse transcription of poly-adenylated mRNA was performed inside each droplet. The cDNA libraries were then completed in a single bulk reaction using the10X Genomics Chromium NextGEM Single Cell V(D)J Reagent Kit and enriched for B cell receptor sequences using the VDJ primers provide in the kit. Following PCR purification, fragment DNA libraries were prepared according to the 10X Genomics protocol. The V(D)J enriched libraries were sequenced to at least 5,000 reads per cell on the Illumina NextSeq 500 instrument. Demultiplexing, barcode processing, alignment and gene counting were performed using the 10X Genomics CellRanger V(D)J (v.3.1.0) software.

$B C R$ reads sequenced by $V(D) J$ assay were aligned to $G R C h 38$ reference transcriptome using Cell Ranger VDJ (v.3.1.0, 10X Genomics). BCR heavy and light chains were assembled and annotated using cellranger vdj with --reference $=$ refdata-cell ranger-vdj-GRCh38-alts-ensembl-3.1.0 to determine clonotypes. Recombinant dimeric IgA antibodies were produced by Genscript. In brief, corresponding DNA sequences for immunoglobulin heavy chain, light chain and $\mathrm{J}$ chain were synthesized, and the complete sequence was subcloned into pcDNA3.4 vector and expressed in HD $293 \mathrm{~F}$ cells. Dimeric IgA1 antibodies were eluted from cell culture supernatants. Molecular weight and purity were analysed by SDS-PAGE and high-performance liquid chromatography.

\section{Statistics and reproducibility}

Unless mentioned otherwise, all data are presented as mean with s.e.m. Two-tailed $t$-tests (unpaired and paired, as appropriate) were performed between two groups, and one-way ANOVA were performed for comparisons between more than two groups, unless indicated otherwise.
Pearson correlation analysis was performed for correlation analysis. To compare $\operatorname{IgA}^{+}$and $\operatorname{lgG}^{+}$or $\operatorname{lgM}^{+}$cell percentage in $\mathrm{B}$ cells, plasmablasts and plasma cells, two-way ANOVA followed by Dunnett's ad hoc tests for multiple comparison was performed on arcsine-transformed percentage data (IgA versus IgG or IgM, $P$ value $=1 \times 10^{-10}$ ). Analyses were carried out in Graph Pad Prism (v.7.0) or R (v.3.6.1) software. A significance threshold 0.05 for $P$ values was used. Box plots were generated using gglot 2 in $\mathrm{R}$ (3.6.1) with following parameters: horizontal black lines with each box present median values; boxes extend from 25th to 75th percentile of values; whiskers extend to a maximum of $1.5 \times$ interquartile range ( 75 th percentile- 25 th percentile) beyond the boxes; the lowest dots are the minimum values and highest dots are the maximum values for each box. All experiments are represented by several biological replicates or independent experiments, unless otherwise mentioned. The number of replicates per experiment is indicated in the legends. Bar plots are mean \pm s.e.m. with overlaid data points representing independent experiments or replicates. All western blot analyses were independently replicated at least two times in case of human-derived specimens and three times in case of in vitro experiments, with similar results. Co-immunoprecipitation experiments were independently replicated a minimum two times with similar results. For all multiplex immunohistochemistry of TMAs, individual tumours have replicated cores (2-6, from different areas of the tumour) on the TMAs, and averaged quantification values from replicated cores were used. The TMA slides were scanned twice, analysed, and used with $<1 \%$ data variation. CRSIPR-mediated knockdown experiments were repeated three times independently. Additional information and test results of statistical analysis are provided in the figure legends and Supplementary Tables 1-12. No sample size calculations were performed before the study for human specimens. For most functional in vitro analyses, sample sizes were chosen on the basis of the availability of target cells. Mouse experiments used at least five mice per group per experiments. Because this study focuses on ovarian cancer, only female mice were included in the experimental design. The experiments were not randomized. HGSOC tumour and ascites specimens were obtained from de-identified patients and were not randomized. Peripheral blood mononuclear cells from de-identified donors without cancer were acquired and analysed. Mice were not intentionally randomized. Tumour volumes in mice were measured using code names on the cages and ear tags, instead of specific information about the treatments that the mice received. Apart from this, no blinding method was used for mouse studies. RNA-seq, BCR sequencing, multiplex immunohistochemistry quantifications, fluorescence microscopy quantifications or LC-MS/MS were performed with unidentifiable demarcation. In case of in vitro experiments, samples were often assigned code numbers to facilitate blinded flow cytometry, microscopy and luciferase assay. After all data were collected, the results were analysed and decoded. For analysis of human specimens, blinding is not applicable as no interventions were tested.

\section{Reporting summary}

Further information on research design is available in the Nature Research Reporting Summary linked to this paper.

\section{Data availability}

The RNA-seq data and single-cell BCR sequencing data related to this study are available at the NCBI Gene Expression Omnibus under accession number GSE146820. The mass spectrometry proteomics data are available in PRIDE with identifier PXD018079. Molecular and clinical data from The Cancer Genome Atlas for ovarian serous cystadenocarcinoma (OV) are available at the cBio Cancer Genomics Portal (http:// www.cbioportal.org/), Broad Firehose website (https://gdac.broadinstitute.org/) and Genomic Data Commons Data Portal (https:// portal.gdc.cancer.gov/). The datasets generated during the current 
study are available from the corresponding author upon reasonable request. Source data are provided with this paper.

22. Bao, Y. et al. Origin, methods, and evolution of the three nurses' health studies. Am. J. Public Health 106, 1573-1581 (2016).

23. Vitonis, A. F., Titus-Ernstoff, L. \& Cramer, D. W. Assessing ovarian cancer risk when considering elective oophorectomy at the time of hysterectomy. Obstet. Gynecol. 117, 1042-1050 (2011).

24. Dobin, A. et al. STAR: ultrafast universal RNA-seq aligner. Bijoinformatics 29, 15-21 (2013).

25. Martin, M. Cutadapt removes adapter sequences from high-throughput sequencing reads. EMBOnet J. 17, 10-12 (2011).

26. Liao, Y., Smyth, G. K. \& Shi, W. featureCounts: an efficient general purpose program for assigning sequence reads to genomic features. Bioinformatics 30, 923-930 (2014).

27. Love, M. I., Huber, W. \& Anders, S. Moderated estimation of fold change and dispersion for RNA-seq data with DESeq2. Genome Biol. 15, 550 (2014).

28. Subramanian, A. et al. Gene set enrichment analysis: a knowledge-based approach for interpreting genome-wide expression profiles. Proc. Natl Acad. Sci. USA 102, 15545-15550 (2005)

29. Ashburner, M. et al. Gene Ontology: tool for the unification of biology. Nat. Genet. 25, 25-29 (2000).

30. Cox, J. \& Mann, M. MaxQuant enables high peptide identification rates, individualized p.p.b.-range mass accuracies and proteome-wide protein quantification. Nat. Biotechnol. 26, 1367-1372 (2008).

31. Anders, S., Pyl, P. T. \& Huber, W. HTSeq-a Python framework to work with high-throughput sequencing data. Bioinformatics 31, 166-169 (2015).

Acknowledgements Support for shared resources was provided by a Cancer Center Support Grant (CCSG) P3O-CA076292 to H. Lee Moffitt Cancer Center. We thank the Analytic Microscopy Core, CLIA Tissue Imaging Laboratory, Molecular Genomics, Proteomics, Flow Cytometry and Biostatistics and Bioinformatics Shared Resources for support; and J. Cleveland for his critical review of this manuscript. This study was supported by R01CA157664, R01CA124515, R01CA178687, R01CA211913 and U01CA232758 to JRCG; RO1CA184185 to P.C.R. K.K.P. was supported by T32CA009140 and The American Cancer Society Postdoctoral Fellowship. We thank the participants and staff of the NHS, NHSII and NECC for their contributions to the collection of tissues from the NHS, NHSII and NECC, which were supported by National Institutes of Health Award Numbers UM1 CA186107, UM1 CA176726, P01 CA087969, R01 CA054419, and R35 CA197605; we also acknowledge the following cancer registries: $A L, A Z, A R, C A, C O, C T, D E, F L, G A, I D, I L, I N, I A, K Y, L A, M E, M D, M A, M I, N E, N H, N J$, $\mathrm{NY}, \mathrm{NC}, \mathrm{ND}, \mathrm{OH}, \mathrm{OK}, \mathrm{OR}, \mathrm{PA}, \mathrm{RI}, \mathrm{SC}, \mathrm{TN}, \mathrm{TX}, \mathrm{VA}, \mathrm{WA}$ and WY.

Author contributions S.B. designed, performed and analysed most of the experiments and co-wrote the manuscript; G.M. performed and analysed staining and imaging, immunoblotting and part of the in vivo experiments; K.K.P., C.M.A., T.L.C., R.A.C. and C.M.H. performed part of the in vivo experiments, processed and stored clinical specimens, and critically reviewed ongoing results and interpretations, as well as the manuscript and the response to the reviewers; C.D.G. and A.R.A. contributed to the design of the study and participated in the analyses and interpretation of all histological analyses; N.S., K.L.T., M.K.T. and S.S.T. provided annotated clinical cohorts, supervised epidemiological analyses and interpreted the results of histological analyses; J.T.-T. and D.M. provided support for the procurement of additional clinical samples and participated in the optimization of the immunofluorescence experiments; K.E.R. and J.A.M. performed in vivo treatments and provided technical guidance; C.M. performed multiplex immunohistochemistry experiments and supported the interpretation of the results; A.B. and R.M.W. provided clinical samples and expertise, and participated in the preparation of the manuscript and the response to the reviewers; X.Y. performed all bioinformatical analyses and contributed to writing the manuscript; P.C.R. contributed to the design of the study, participated in the interpretation of the results throughout all phases of the study, and contributed to writing the manuscript and designing the experiments to respond to reviewers; and J.R.C.-G. oversaw and designed the study and experiments, analysed data and co-wrote the manuscript.

Competing interests J.R.C.-G. has stock options and sponsored research, and receives consulting fees from Compass Therapeutics and Anixa Biosciences. He also receives consulting fees from Leidos. R.M.W. reports grants and personal fees from Merck, personal fees from Tesaro/GSK, personal fees from Genentech, personal fees from Legend Biotech, personal fees from AbbVie, personal fees from AstraZeneca, grants and stock from Ovation Diagnostics, personal fees from Clovis Oncology and personal fees from Regeneron, outside the submitted work. Otherwise, the authors do not have any conflicts of interest.

Additional information

Supplementary information The online version contains supplementary material available at https://doi.org/10.1038/s41586-020-03144-0.

Correspondence and requests for materials should be addressed to J.R.C.-G.

Peer review information Nature thanks George Coukos, Tyler J. Curiel and the other,

anonymous, reviewer(s) for their contribution to the peer review of this work. Reprints and permissions information is available at http://www.nature.com/reprints. 


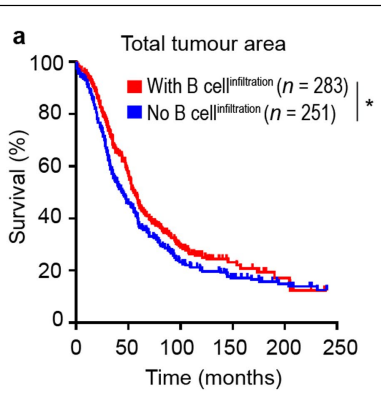

d
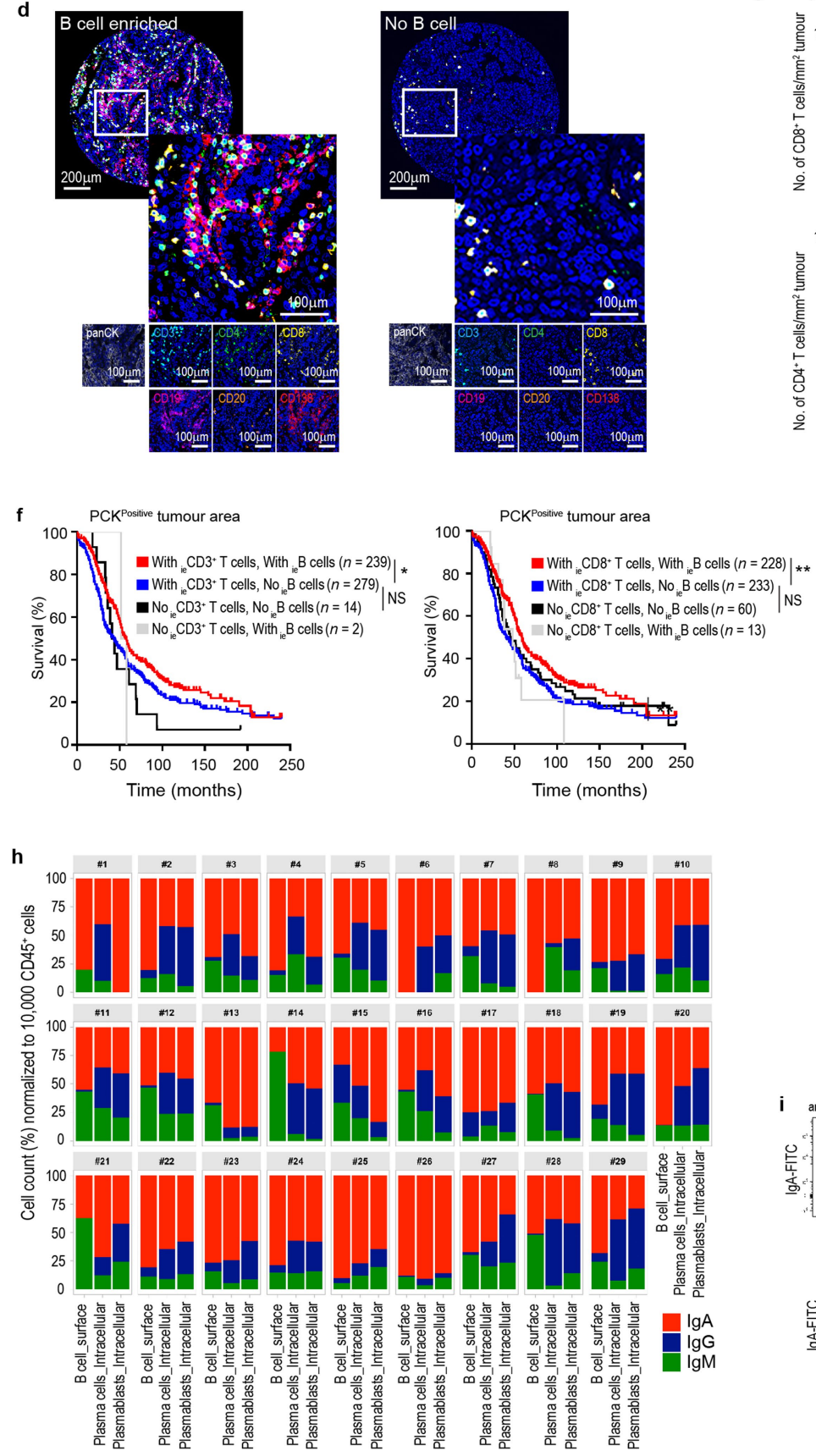

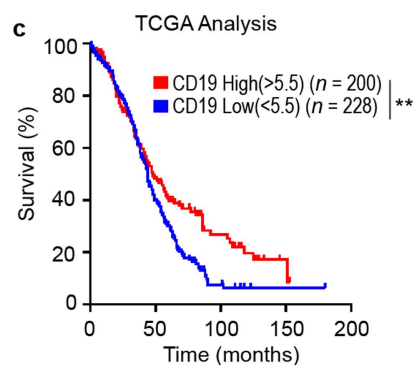

e

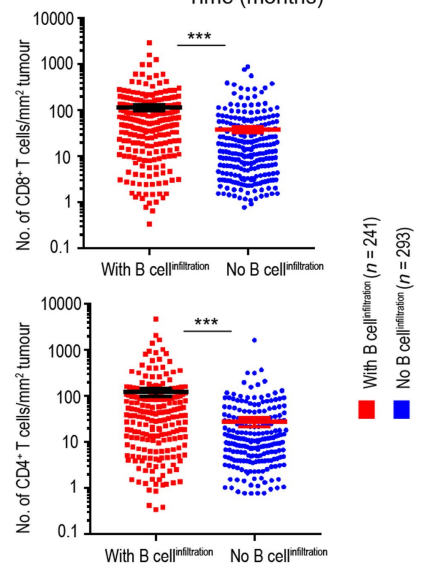

g Viable $C D 45^{+} \mathrm{CD} 3 \mathrm{CD} 19^{+} \mathrm{CD} 20^{+}$

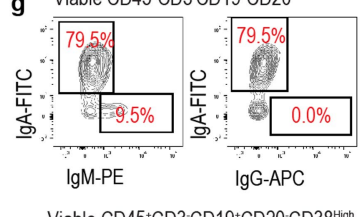

Viable CD45+CD3-CD19+CD20-CD38 High $^{\text {Hig }}$

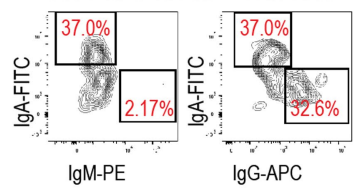

Viable CD45+CD3-CD19+'-CD20-CD138+

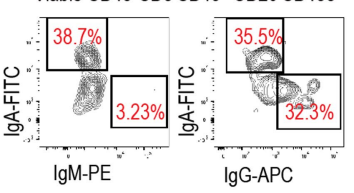

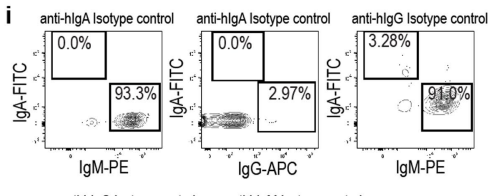

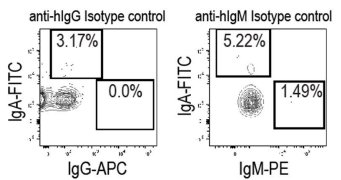

Extended Data Fig. 1|See next page for caption. 
Extended Data Fig. 1 | Isotype-switched humoral responses are associated with better outcome and denser T cell infiltration in HGSOC. $a, b$, Overall survival associated with the presence of $\mathrm{CD} 19^{+} \mathrm{B}$ cells within the total tumour area $(P=0.04)$ (a) or specifically in the $\mathrm{PCK}^{+}$epithelial tumour islets $(P=0.0085)$ (b), in HGSOC as assessed by multiplex immunohistochemistry of TMAs corresponding to 534 patients with HGSOC combined from the NECC $(n=180)$, NHS $(n=261)$ and MCC $(n=93)$. B cell infiltration is defined as the presence of $\mathrm{CD} 19^{+}$cells on any of the duplicate sections analysed for each tumour. c, Survival outcome associated with the expression of CD19 in 428 annotated HGSOCs in TCGA datasets $(P=0.0091) .{ }^{*} P \leq 0.05,{ }^{* *} P \leq 0.01$, two-sided log-rank (Mantel-Cox) test.d, Representative staining of the association between the accumulations of T and B cells at tumour beds $(n=534)$. Scale bar, $200 \mu \mathrm{m}$ or $100 \mu \mathrm{m}$, as indicated. e, Accumulation of $\mathrm{CD}^{+}$(top) and $\mathrm{CD}^{+}$(bottom) T cells in the $\mathrm{PCK}^{+}$tumour islets are associated with the presence of $\mathrm{B}$ cells. $\mathrm{CD} 8$ : with versus without $\mathrm{B}$ cell infiltration, $P<0.0001 ; \mathrm{CD} 4$ : with versus without $\mathrm{B}$ cell infiltration, $P<0.0001$. Data are mean \pm s.e.m. ${ }^{* * *} P \leq 0.001$, unpaired two-tailed $t$-test.f, Overall survival associated with the presence of intra-epithelial CD19 ${ }^{+}$ B cells and total intra-epithelial $\mathrm{CD}^{+}{ }^{+} \mathrm{T}$ cells (left) or intra-epithelial CD3 ${ }^{+}$ $\mathrm{CD}^{+} \mathrm{T}$ cells (right) within ovarian carcinomas, as assessed by multiplex immunohistochemistry of TMAs corresponding to 534 patients with HGSOC combined from the NECC $(n=180)$, NHS $(n=261)$ and MCC $(n=93)$. With intra-epithelial CD3 T cells and with-intra-epithelial B cells versus with intraepithelial CD3 T cells and no intra-epithelial B cells, $P=0.0123$; With intraepithelial CD3 $T$ cells and no intra-epithelial $B$ cells versus no intra-epithelial CD3 T cells and no intra-epithelial $B$ cells, $P=0.0572$; with intra-epithelial CD8 $T$ cells and with intra-epithelial $B$ cells versus with intra-epithelial CD8 T cells and no intra-epithelial B cells, $P=0.0035$; with intra-epithelial CD8 T cells and no intra-epithelial $B$ cells versus no intra-epithelial CD8 T cells and no intraepithelial $\mathrm{B}$ cells, $P=0.4113$. B cell and $\mathrm{T}$ cell infiltration are defined as the presence of $\mathrm{CD} 19^{+}$or $\mathrm{CD} 3^{+}$cells on any of the duplicate sections analysed for each tumour. ${ }^{*} P \leq 0.05,{ }^{* *} P \leq 0.01$, NS, not significant, two-sided log-rank (Mantel-Cox) test.g, Representative FACS analysis of immunoglobulin isotypes on the surface of $B$ cells infiltrating freshly dissociated human HGSOCs $(n=29)$. h, Bar graphs representing tumour-wise $(n=29)$ FACS analysis comparison of percentages of each $^{+}$cells among total Ig $^{+} B$ cells $\left(\mathrm{CD} 45^{+} \mathrm{CD}^{-} \mathrm{CD}^{-} 9^{+} \mathrm{CD} 2 \mathrm{O}^{+}\right.$), plasma cells (intracellular in $\mathrm{CD}^{-} 5^{+} \mathrm{CD}^{-} \mathrm{CD}^{-} 9^{+} \mathrm{CD} 20^{-}$ $\mathrm{CD} 138^{+}$and $\mathrm{CD}^{-} 5^{+} \mathrm{CD}^{-} \mathrm{CD}^{-} 9^{-} \mathrm{CD} 20^{-} \mathrm{CD} 138^{+}$) and plasmablasts (intracellular in $\mathrm{CD}^{2} 5^{+} \mathrm{CD} 3^{-} \mathrm{CD} 19^{+} \mathrm{CD} 20 \mathrm{CD}^{-} 8^{\text {high }}$ ), normalized to 10,000 viable $\mathrm{CD} 45^{+}$cells. i, FACS dot plots from analysis of IgA, IgG and IgM antibodies, and respective isotype controls, in human peripheral blood mononuclear cells, to evaluate the fidelity of the antibodies used. 

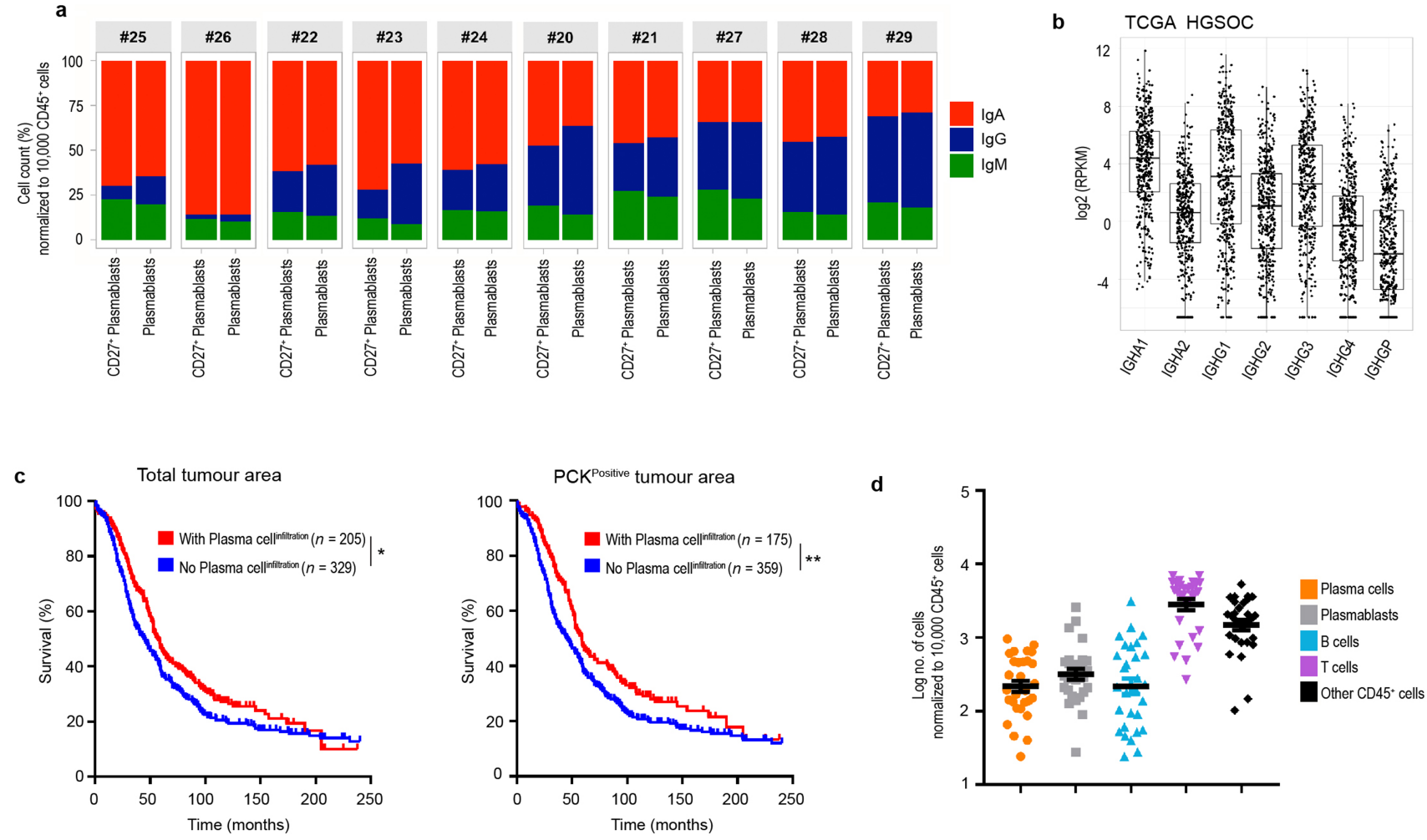

e
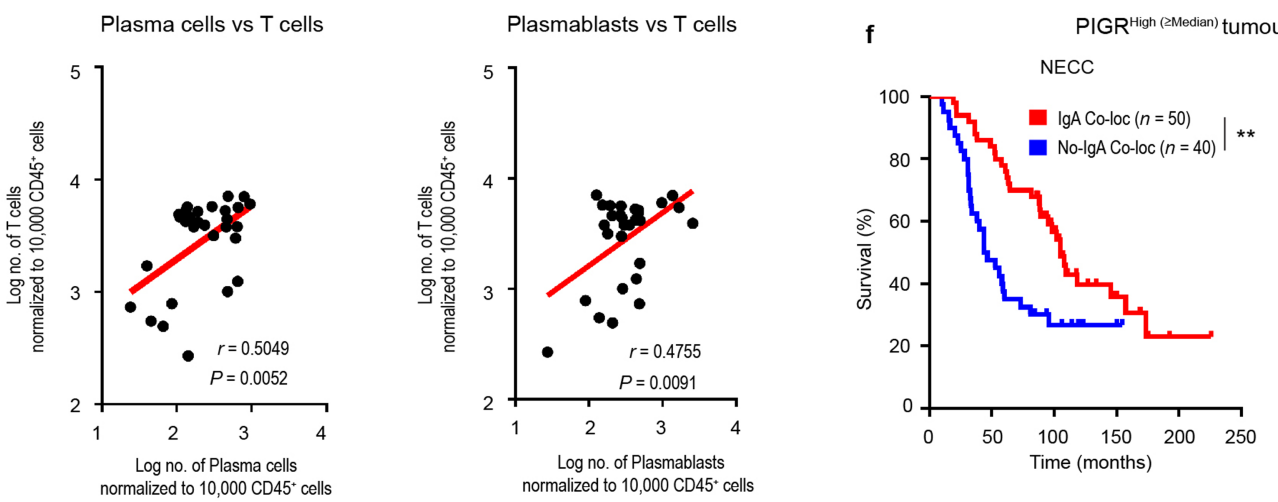

MCC

g

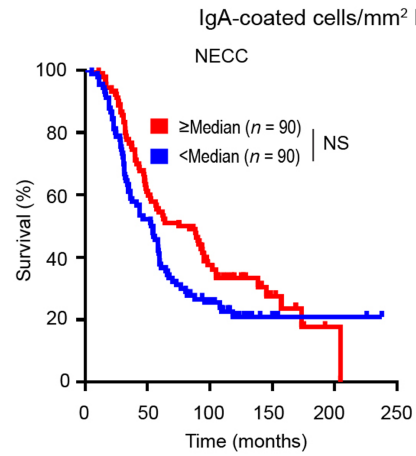

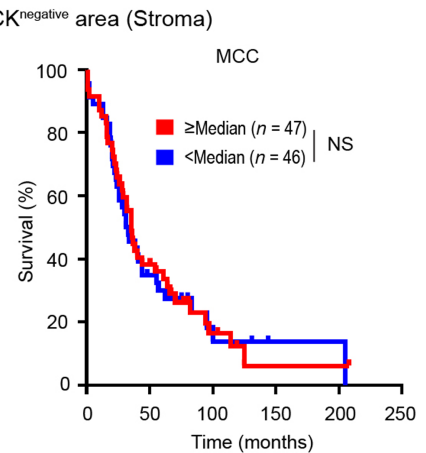
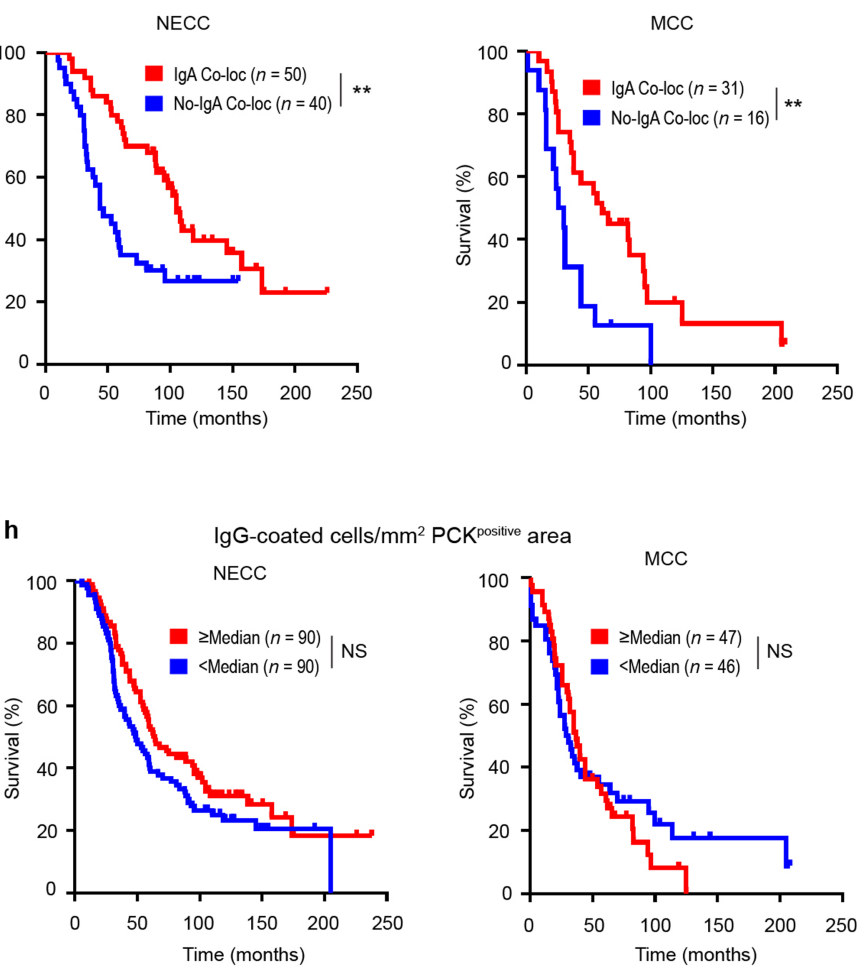

Extended Data Fig. 2 |See next page for caption.

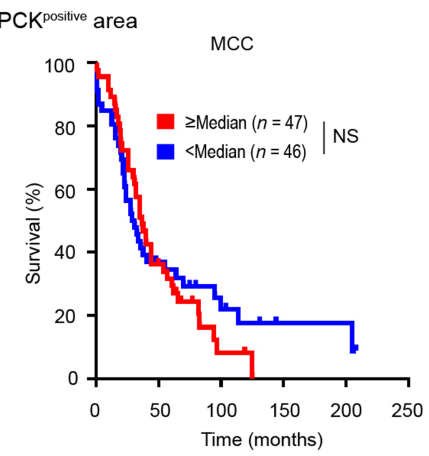


Extended Data Fig. 2 | IgA coating of tumour cells is associated with a better outcome in HGSOC. a, Bar graphs representing tumour-wise FACS analysis $(n=10)$ comparison of percentages of each Ig $^{+}$cells among total Ig $^{+}$ plasmablasts (intracellular in $\mathrm{CD}^{+} 5^{+} \mathrm{CD} 3^{-} \mathrm{CD} 19^{+} \mathrm{CD} 20^{-} \mathrm{CD} 38^{\text {high }}$ ) and $\mathrm{CD} 27^{+}$ plasmablasts (intracellular in $\mathrm{CD} 45^{+} \mathrm{CD} 3^{-} \mathrm{CD} 19^{+} \mathrm{CD} 20^{-} \mathrm{CD} 38^{\text {high }} \mathrm{CD} 27^{+}$), normalized to 10,000 viable $\mathrm{CD} 45^{+}$cells. b, Relative abundances of IgH chains on the basis of TCGA transcriptional analyses $(n=430)$ for each immunoglobulin heavy chain gene. Abundances are shown in $\log _{2}$-transformed reads per kilobase of transcripts (RPKM) values, which corrects for both gene length and sequencing depth. Details of box plots are in Methods. c, Overall survival associated with the presence of $\mathrm{CD} 19^{+} \mathrm{CD} 138^{+}$plasma cells within the total tumour area $(P=0.0285)$ (left) or specifically in the $\mathrm{PCK}^{+}$epithelial tumour islets $(P=0.0053)$ (right), in HGSOC as assessed by multiplex immunohistochemistry of TMAs corresponding to 534 patients with HGSOC combined from the NECC $(n=180)$, NHS $(n=261)$ and MCC $(n=93)$. Plasma cell infiltration is defined as the presence of $\mathrm{CD} 19^{+} \mathrm{CD} 138^{+}$cells on any of the duplicate sections analysed for each tumour. ${ }^{*} P \leq 0.05,{ }^{* *} P \leq 0.01$, two-sided log-rank (Mantel-Cox) test.d, FACS analysis showing number (log-transformed) of plasma cells $\left(\mathrm{CD} 45^{+} \mathrm{CD}^{-} \mathrm{CD} 19^{+} \mathrm{CD} 20^{-} \mathrm{CD} 138^{+}\right.$and $\mathrm{CD} 45^{+} \mathrm{CD}^{-} \mathrm{CD} 19$ $\left.\mathrm{CD} 20^{-} \mathrm{CD} 138^{+}\right)$, plasmablasts $\left(\mathrm{CD} 45^{+} \mathrm{CD} 3^{-} \mathrm{CD} 19^{+} \mathrm{CD} 20^{-} \mathrm{CD} 38^{\text {high }}\right)$, B cells $\left(\mathrm{CD}^{+} 5^{+} \mathrm{CD}^{-} \mathrm{CD}^{-} 9^{+} \mathrm{CD} 2 \mathrm{O}^{+}\right)$, $\mathrm{T}$ cells $\left(\mathrm{CD} 45^{+} \mathrm{CD}^{+}\right)$and other leukocytes $\left(\mathrm{CD} 45^{+} \mathrm{CD}\right.$ $\left.3^{-} \mathrm{CD}^{-} 9^{-} \mathrm{CD} 20^{-} \mathrm{CD} 138^{-}\right)$in HGSOC $(n=29)$. The data are normalized to 10,000 viable $C D 45^{+}$leukocytes. Data are mean \pm s.e.m.e, Graphs showing correlations between log count of T cells and plasma cells (left) (Pearson correlation coefficient $(r)=0.5049$; two-sided nominal $P=0.0052$ ); and between log count of T cells and plasmablasts (right) (Pearson correlation coefficient $(r)=0.4755$; two-sided nominal $P=0.0091)$. All three cell types represent absolute counts normalized to $10,000 \mathrm{CD} 45^{+}$leukocytes.

f, Colocalization of $\operatorname{lgA}$ with $\operatorname{lgR}^{+}$cells (IgA-plgR co-localization $\geq$median) in the $\mathrm{PCK}^{+}$tumour islets is associated with an improved outcome in HGSOC, compared to only plgR ${ }^{\text {high }}$ samples ( $\geq$ median) without IgA colocalization (colocalization $<$ median), in MCC $(P=0.0060)$ and NECC $(P=0.0044)$ cohorts. ${ }^{* *} P \leq 0.01$, two-sided log-rank (Mantel-Cox) test. g, Higher number of IgAcoated cells in the $\mathrm{PCK}^{-}$stromal area (average from duplicated cores) is not associated with an improved outcome in HGSOC, analysed using median IgAcoating threshold in MCC $(P=0.8954)$ and NECC $(P=0.0537)$ cohorts. NS, not significant; two-sided log-rank (Mantel-Cox) test, no multiple comparison adjustment. $\mathbf{h}$, A higher number of IgG-coated cells in the $\mathrm{PCK}^{+}$tumour islets (average from duplicated cores) is not associated with an improved outcome in HGSOC, analysed using median IgG-coating threshold in MCC $(P=0.6350)$ and NECC $(P=0.0731)$ cohorts. NS, not significant; two-sided log-rank (MantelCox) test. 


\section{Article}

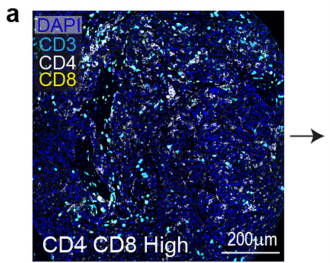

CD4 CD8 High $200 \mu \mathrm{m}$
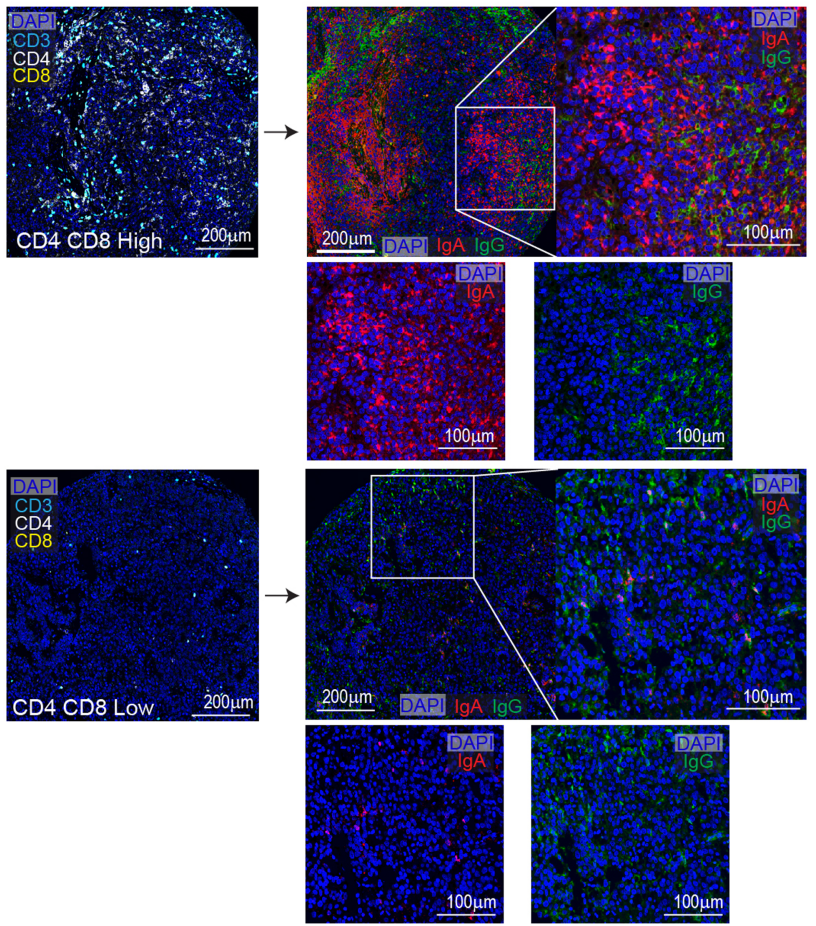

b IgA/lgG-APC, DAPI

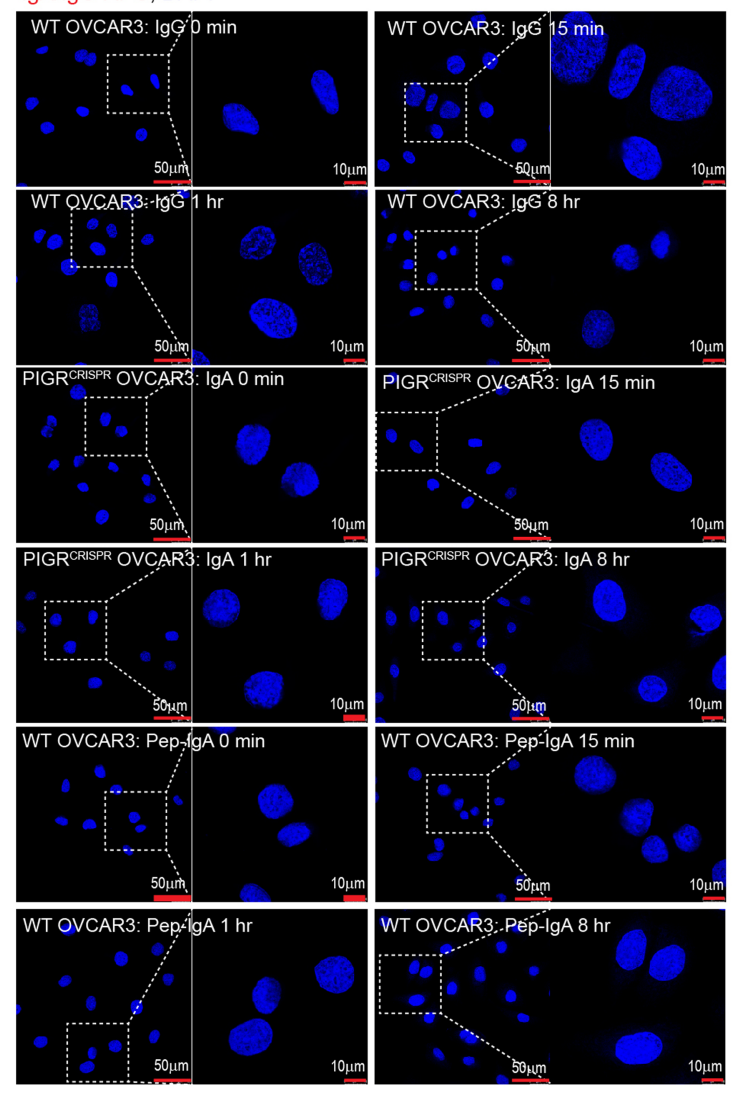

Extended Data Fig. 3 | See next page for caption.
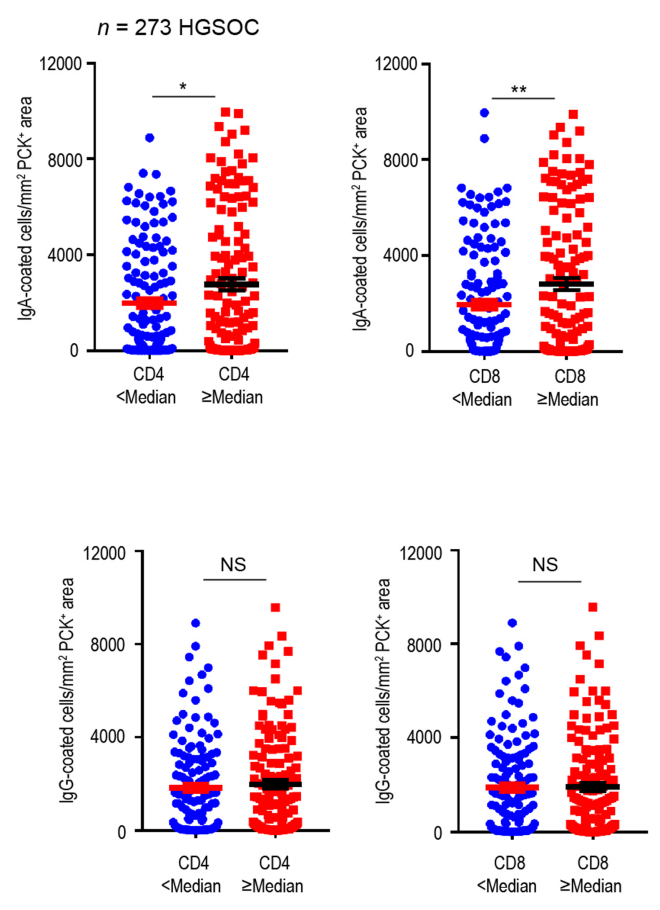

C
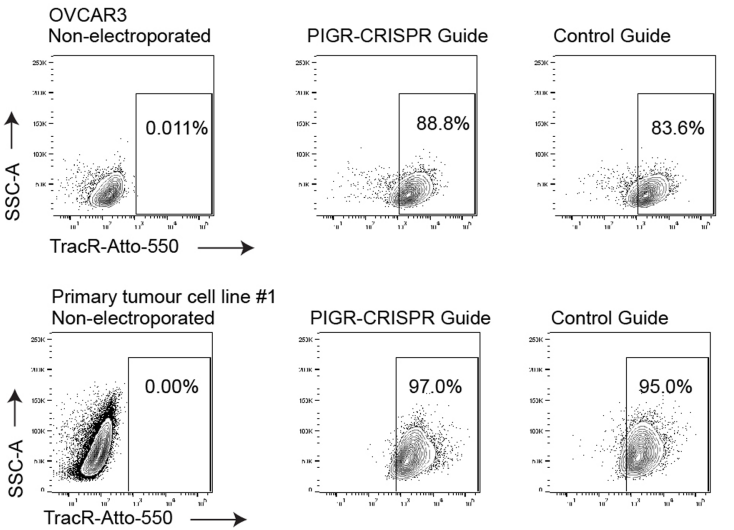

Primary tumour cell line \#2
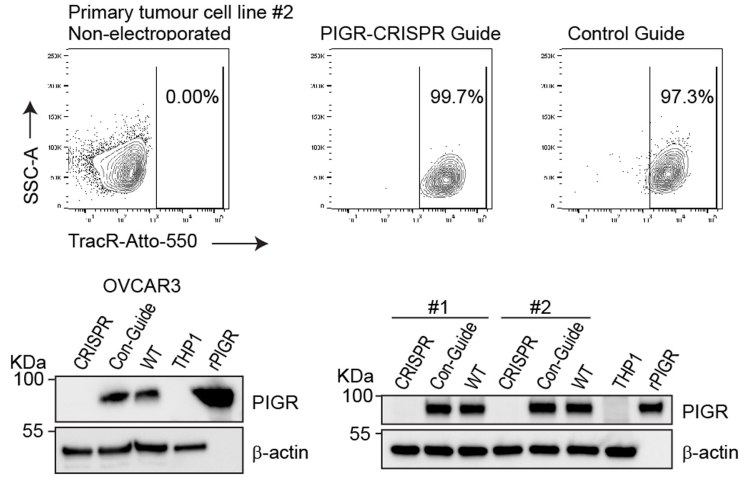
Extended Data Fig. 3 | IgA internalization by HGSOC cells is associated with stronger $T$ cell responses, and is dependent on pIgR-Fc interactions. a, Representative $(n=273)$ IgA and IgG staining in tumours with a high or low density of $\mathrm{CD}^{+}{ }^{+}$and $\mathrm{CD} 8^{+} \mathrm{T}$ cells. Scale bar, $200 \mu \mathrm{m}$ or $100 \mu \mathrm{m}$, as indicated. $\mathrm{CD}^{+}$and $\mathrm{CD} 8^{+} \mathrm{T}$ cell accumulation ( $\geq$ median) is associated with the density of IgA-coated tumour $\left(\mathrm{PCK}^{+}\right)$cells (IgA: $\mathrm{CD} 4<$ median versus $\mathrm{CD} 4 \geq$ median, $P=0.0209$; IgA: $\mathrm{CD} 8<$ median versus $\mathrm{CD} 8 \geq$ median, $P=0.0087$ ); but not associated with IgG-coated tumour $\left(\mathrm{PCK}^{+}\right)$cells $(\mathrm{IgG}: \mathrm{CD} 4<$ median versus CD4 $\geq$ median, $P=0.4451$; IgG: $\mathrm{CD} 8<$ median versus $C D 8 \geq$ median, $P=0.8304$ ). Data are mean \pm s.e.m. ${ }^{*} P \leq 0.05,{ }^{* *} P \leq 0.01$, NS, not significant; unpaired twotailed $t$-test.b, Representative confocal microscopy images of fluorescently
(APC) labelled whole or pepsinized irrelevant IgA- or IgG-binding and internalization in pIgR $^{+}(\mathrm{WT})$ or PIGR-ablated (PIGR ${ }^{\mathrm{CRIPR}}$ ) OVCAR3 cells after $0 \mathrm{~min}, 15 \mathrm{~min}, 1 \mathrm{~h}$ or $8 \mathrm{~h}$ of incubation. Experiments were repeated three times. Scale bar, $50 \mu \mathrm{m}$ or $10 \mu \mathrm{m}$, as indicated.c, FACS dot plots showing electroporation efficiency in pIgR-CRSIPR-guide (centre) or control-guide electroporated cells (right), compared to nonelectroporated OVCAR3 or primary HGSOC cells $(n=2)$ (left). Experiments were repeated three times. Western blots confirmed PIGR ablation in OVCAR3 and primary HGSOC cells ( $n=2$, repeated twice). THP1 cells were used as a negative control and recombinant pIgR (rPIGR) was used as a positive control. WT, wild type (non-electroporated cells). 


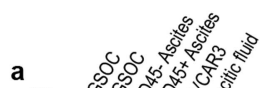
In:IgA, WB: IgA
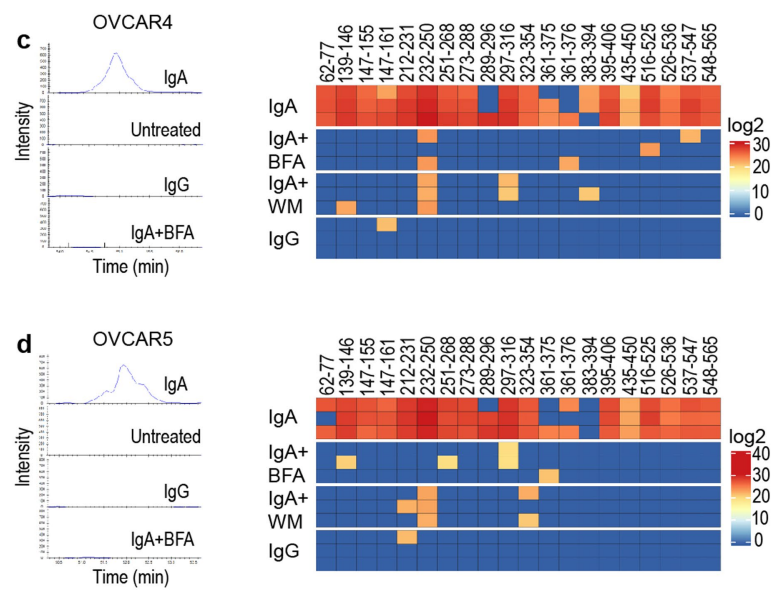

e Primary tumour derived ovarian cancer cells
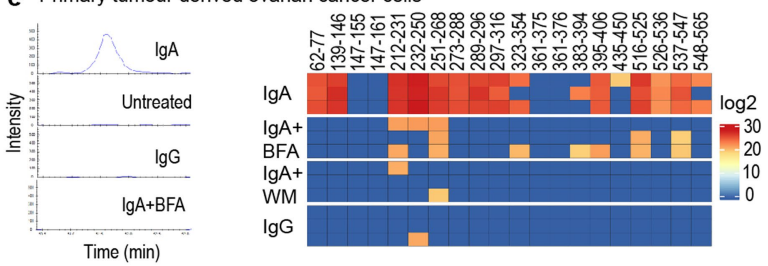

h

IgA-treated PIGR ${ }^{\text {CRISPR }}$ OVCAR3
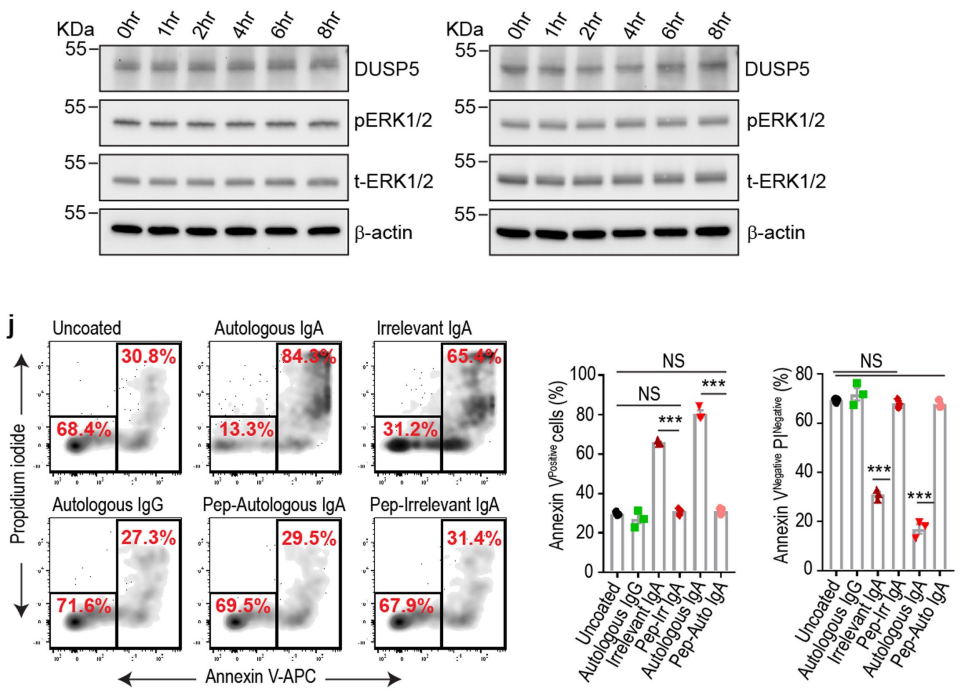
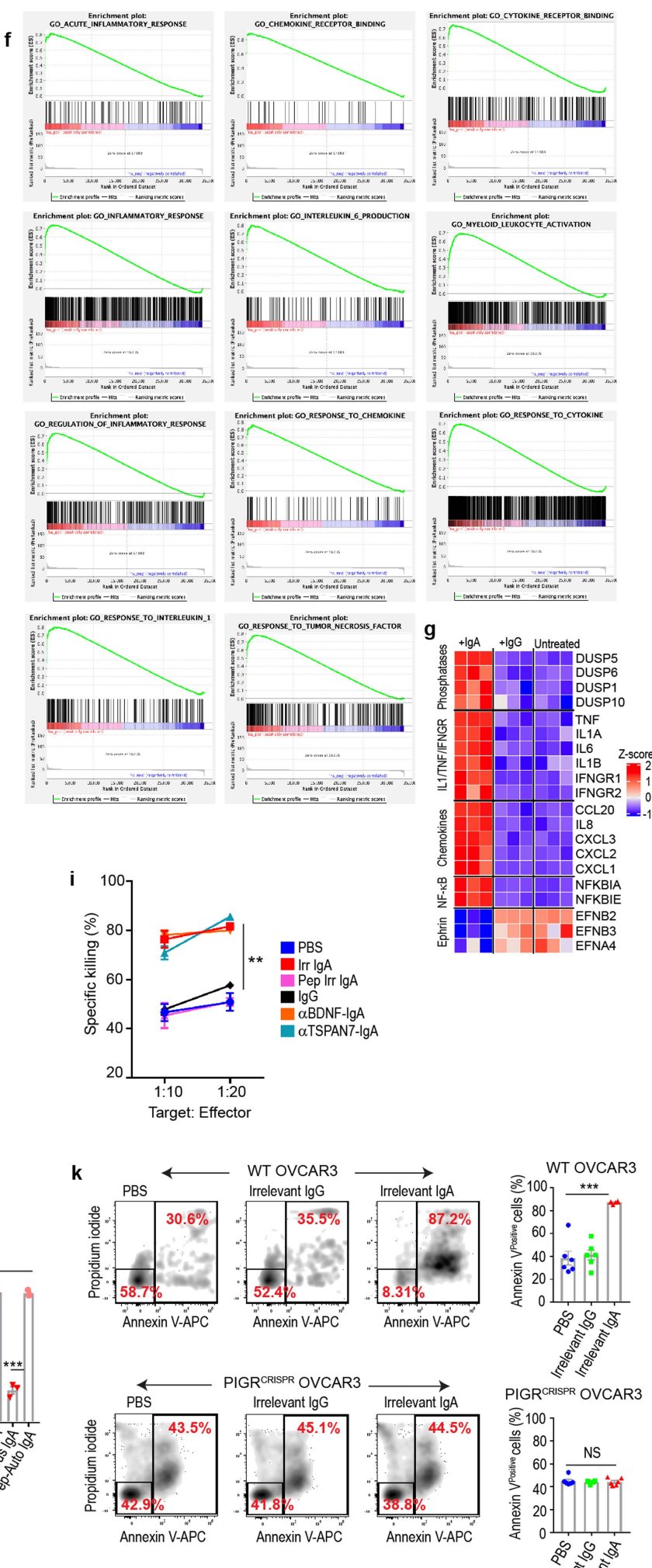

Extended Data Fig. 4 |See next page for caption. 
Extended Data Fig. 4 | IgA transcytosis through HGSOC cells has substantial anti-tumour effects and sensitizes tumour cells to cytolytic killing by T cells. a, Immunoblots showing pIgR co-immunoprecipitation with IgA, using nondenaturing lysates from two HGSOCs, $\mathrm{CD}^{+} 5^{+}$and $\mathrm{CD}^{-} 5^{-}$cells isolated from human ovarian cancer ascites, ascitic fluid and OVCAR3 cells (negative control). Inputs were immunoblotted using $1 \%$ of the amount of lysate used for the co-immunoprecipitation. The experiments were repeated three times. b, OVCAR 3 cells were incubated with $0.5 \mu \mathrm{g} \mathrm{ml}^{-1}$ of control IgA or $\operatorname{IgG}$ for $8 \mathrm{~h}$ in serum-free medium in the presence of wortmannin $(1 \mu \mathrm{M})$, brefeldin $\mathrm{A}\left(1 \mu \mathrm{g} \mathrm{ml}^{-1}\right)$ or vehicle, and supernatants were subjected to mass spectrometry. The amino acids 62-77 fragment of plgR was found only after incubation with IgA (repeated three times). c-e, OVCAR4 (c), OVCAR5 (d) and primary HGSOC (e) tumour cells were incubated with $0.5 \mu \mathrm{g} \mathrm{ml}^{-1}$ of irrelevant IgA or IgG for $8 \mathrm{~h}$ in serum-free medium in the presence of wortmannin $(1 \mu \mathrm{M})$, brefeldin A $\left(1 \mu \mathrm{g} \mathrm{ml}^{-1}\right)$ or vehicle, and supernatants were then subjected to mass spectrometry (left). Right, heat map of all peptides of the extracellular domain of plgR $(n=3)$. BFA, brefeldin A. WM, wortmannin. f, g, GSEA enrichment plots (h) and heat maps (g) using normalized gene expression from RNA-seq analysis from OVCAR3 cells with irrelevant IgA $\left(0.5 \mu \mathrm{g} \mathrm{ml}^{-1}\right), \operatorname{lgG}\left(0.5 \mu \mathrm{g} \mathrm{ml}^{-1}\right)$ or no treatment for $24 \mathrm{~h}(n=3$ experiments). $\mathrm{h}$, No change in the protein levels of DUSP5, total ERK1/2 or phospho-ERK1/2 after vehicle (PBS) treatment of plgR ${ }^{+}$ (WT) OVACR3 cells (left) or after IgA treatment of PIGR-ablated OVACR3 cells (right), incubated up to $8 \mathrm{~h}$. The experiments were repeated three times. i, Dose-dependent cytotoxic killing of OVCAR3 cells by FSH-targeted chimeric receptor T cells is augmented by co-incubation with $0.5 \mu \mathrm{g} \mathrm{ml}^{-1}$ of irrelevant IgA, anti-TSPAN7-IgA or anti-BDNF-IgA compared to IgG, pepsinized irrelevant $\operatorname{lgA}$ or PBS. $n=3$ per group. ${ }^{* *} P \leq 0.01$, unpaired two-tailed $t$-test. j, Cytotoxic killing of autologous $\mathrm{CD} 45^{-} \mathrm{EpCAM}^{+}$tumour cells (with corresponding decrease of annexin $\mathrm{V}^{-}$propidium iodide ( $\left.\mathrm{PI}\right)^{-}$viable cells) by autologous T cells (added at 1:1 ratio) is augmented by co-incubation with $0.5 \mu \mathrm{g} \mathrm{ml}^{-1}$ of autologous IgA or irrelevant IgA but not with autologous IgG, pepsinized autologous or irrelevant IgA, as compared to uncoated cells $(n=3)$. Annexin $\mathrm{V}^{+}$: irrelevant IgA versus pepsinized irrelevant IgA, $P<0.0001$; autologous IgA versus pepsinized autologous IgA, $P<0.0001$; uncoated versus pepsinized irrelevant IgA, $P=0.3769$; uncoated versus pepsinized autologous IgA, $P=0.2208$. Annexin $\mathrm{V}^{-} \mathrm{PI}^{-}$cells: irrelevant IgA versus pepsinized irrelevant IgA, $P<0.0001$; autologous IgA versus pepsinized autologous IgA, $P<0.0001$; uncoated versus pepsinized irrelevant IgA, $P=0.3329$; uncoated versus pepsinized autologous $\operatorname{IgA}, P=0.1916$. Data are mean \pm s.e.m. ${ }^{* * *} P \leq 0.001$, NS, not significant; unpaired two-tailed $t$-test. $\mathbf{k}$, Cytotoxic killing of plgR $^{+}$OVCAR3 cells, but not pIgR ${ }^{\text {CRISPR }}$ OVCAR3 cells, by FSH-targeted chimeric receptor T cells (added at 1:1 ratio) is augmented by co-incubation with $0.5 \mathrm{\mu g} \mathrm{ml}^{-1}$ of irrelevant IgA compared to IgG-coated or uncoated cells $(n=6)$. Wild-type OVCAR3: PBS or irrelevant IgG versus irrelevant IgA, $P<0.0001$. plgR $^{\text {CRISPR }}$ OVCAR3: PBS versus irrelevant $\operatorname{IgA}, P=0.7728$; irrelevant $\operatorname{IgG}$ versus irrelevant $\operatorname{lgA}, P=0.9176$. ${ }^{* * *} P \leq 0.001$, NS, not significant; unpaired two-tailed $t$-test. 

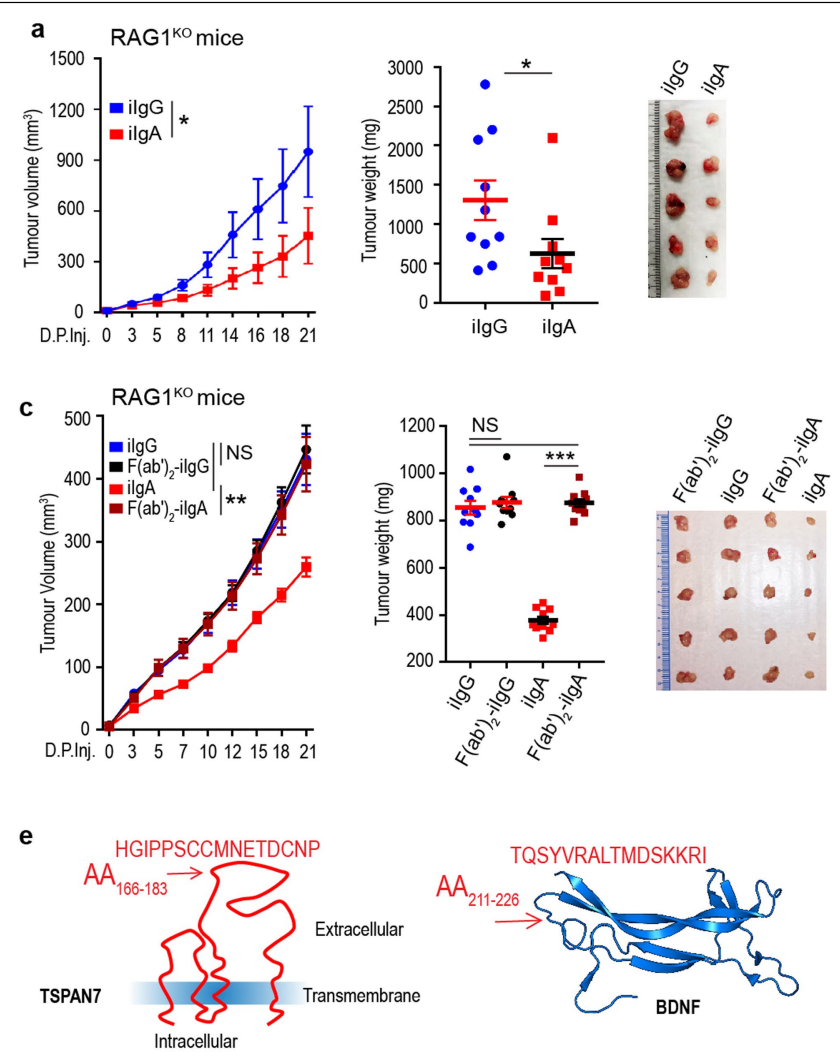

f
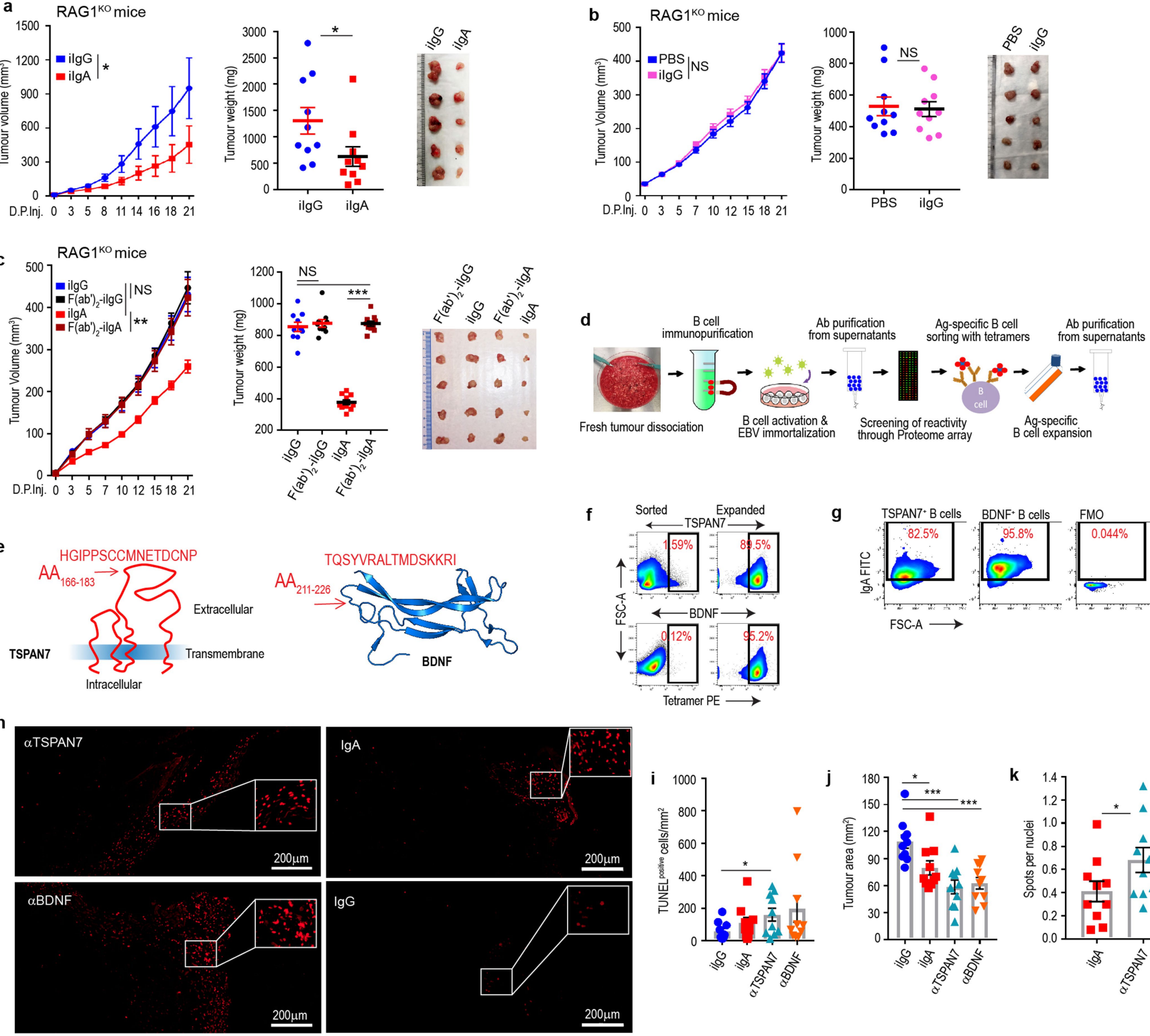

$$
\text { (1) }
$$

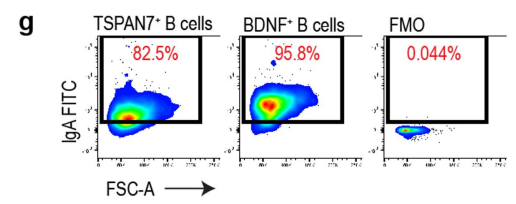

Extended Data Fig. 5 | TSPAN7- and BDNF-specific antibodies produced at tumour beds delay the progression of established tumours.

a, RAG1-deficient mice inoculated subcutaneously with $10^{7}$ OVCAR3 cells received $100 \mu$ g per $20 \mathrm{~g}$ body weight of irrelevant (i) IgA or irrelevant (i)IgG peritumourally at days $7,11,15,19$ and 23 after tumour inoculation. Tumour growth curves (left), tumour weight (centre) and representative differences in tumour volume (right) are shown. Growth curve statistics: ilgG versus ilgA, $P=0.0130$, paired two-tailed $t$-test. Tumour weight statistics, ilgG versus ilgA, $P=0.043$, unpaired two-tailed $t$-test. Data are mean \pm s.e.m. ${ }^{*} P \leq 0.05$. b, Tumour growth curves (left), as well as tumour volume (right) and weight (centre) in OVCAR3-tumour-bearing RAG1-deficient mice receiving irrelevant IgG antibodies or vehicle (PBS). Growth curve statistics: ilgG versus PBS, $P=0.1840$, paired two-tailed $t$-test. Tumour weight statistics: ilgG versus $P B S, P=0.8275$, (unpaired two-tailed $t$-test). Data are mean \pm s.e.m. NS, not significant. c, Tumour growth curves (left), as well as tumour volume (right) and weight (centre) in OVCAR3-tumour-bearing RAG1-deficient mice receiving full-length or pepsinized (Fc-removed) irrelevant IgG or irrelevant IgA antibodies. Curves and tumour weights were pooled from 2 independent experiments ( 10 mice per group in total). Growth curve statistics: ilgA versus $\mathrm{F}\left(\mathrm{ab}^{\prime}\right)_{2}$-ilgA, $P=0.0030$; ilgA versus ilgG, $P=0.0578$; ilgG versus $\mathrm{F}\left(\mathrm{ab}^{\prime}\right)_{2}$-ilgG, $P=0.0547$, paired two-tailed $t$-test. Tumour weight statistics: ilgA versus $\mathrm{F}\left(\mathrm{ab}^{\prime}\right)_{2}$-ilgA, $P<0.0001$;
ilgG versus $\mathrm{F}\left(\mathrm{ab}^{\prime}\right)_{2}-\mathrm{ilgG}, P=0.5585$; ilgG versus $\mathrm{F}\left(\mathrm{ab}^{\prime}\right)_{2}-\mathrm{ilgA}, P=0.5382$, unpaired two-tailed $t$-test. Data are mean \pm s.e.m. ${ }^{* *} P \leq 0.01$, ${ }^{* * *} P \leq 0.001$, NS, not significant. In a-c, growth curves and tumour weights were pooled from 2 independent experiments ( $n=10$ mice per group in total). d, Schematic of the optimized protocol for separating, immortalizing, characterizing and selecting tumour-reactive B cells from HGSOCs. e, Tetramers spanning the indicated loop in BDNF or the extracellular domain of TSPAN7 were used to sort reactive $B$ cells immortalized from ten independent HGSOCs.f, The reactivity of expanded cells was confirmed using the same tetramers. $g$, IgA represents the majority of TSPAN7- or BDNF-reactive B cells sorted from HGSOCs. h, Representative TUNEL (Alexa Fluor 647) staining images in xenograft tumours developed in RAG1-knockout mice. Scale bar, $200 \mu \mathrm{m}$. i, Estimation of TUNEL $^{+}$cells normalized to tumour area. ilgG versus anti-TSPAN7, $P=0.0293$. Data are mean \pm s.e.m. ${ }^{*} P \leq 0.05$, unpaired two-tailed $t$-test.j, Tumour area quantification. ilgG versus ilgA, $P=0.0146$; ilgG versus anti-BDNF, $P=0.0002$; ilgG versus anti-TSPAN7, $P=0.0002$. Data are mean \pm s.e.m. ${ }^{*} P \leq 0.05$, ${ }^{* * *} P \leq 0.001$, unpaired two-tailed $t$-test. $\mathbf{k}$, Quantification of irrelevant IgA and anti-TSPAN7-IgA antibody uptake in OVACR3 xenografts. $P=0.0343$. Data are mean \pm s.e.m. ${ }^{*} P \leq 0.05$, unpaired two-tailed $t$-test. $\ln \mathbf{h}-\mathbf{k}, n=10$ mice per group pooled from 2 independent experiments. 

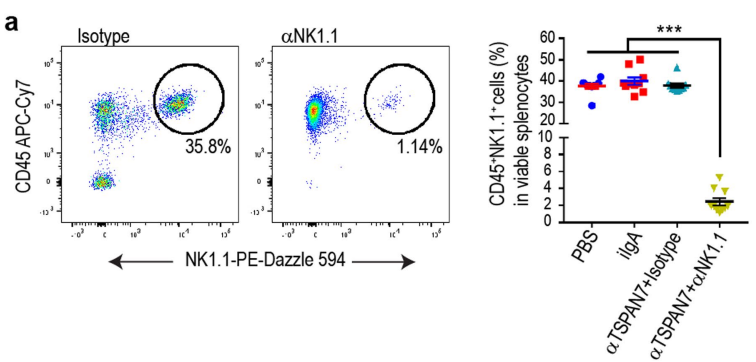

C Viable singlet $\mathrm{CD} 45^{+} \mathrm{CD} 11 \mathrm{~b}^{+}$cells
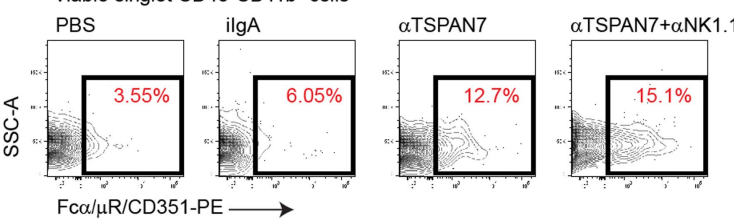

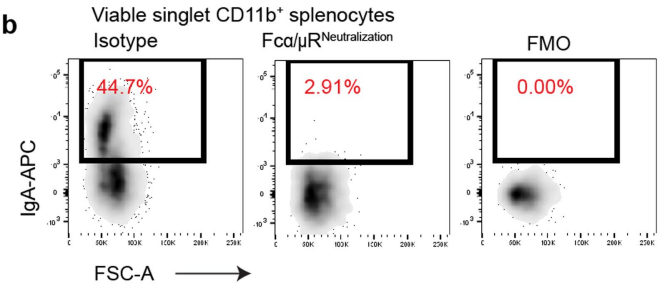

d PIGR ${ }^{\text {CRISPR }}$ tumour NSG mice
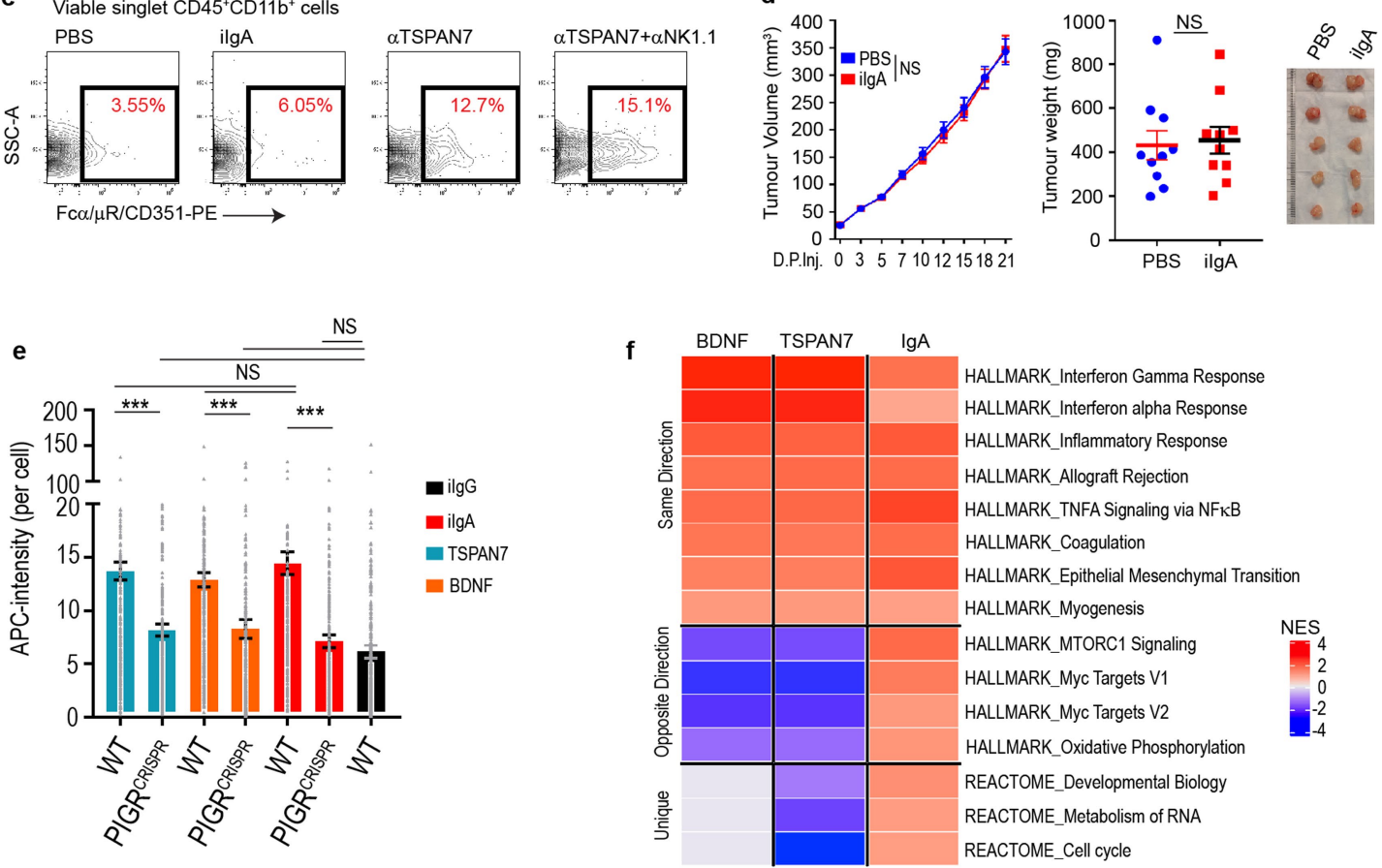

HALLMARK_Interferon Gamma Response HALLMARK_Interferon alpha Response HALLMARK_Inflammatory Response HALLMARK_Allograft Rejection HALLMARK_TNFA Signaling via NFKB HALLMARK_Coagulation HALLMARK_Epithelial Mesenchymal Transition HALLMARK_Myogenesis HALLMARK_MTORC1 Signaling HALLMARK_Myc Targets V1 HALLMARK_Myc Targets V2 HALLMARK_Oxidative Phosphorylation REACTOME_Developmental Biology REACTOME_Metabolism of RNA REACTOME_Cell cycle
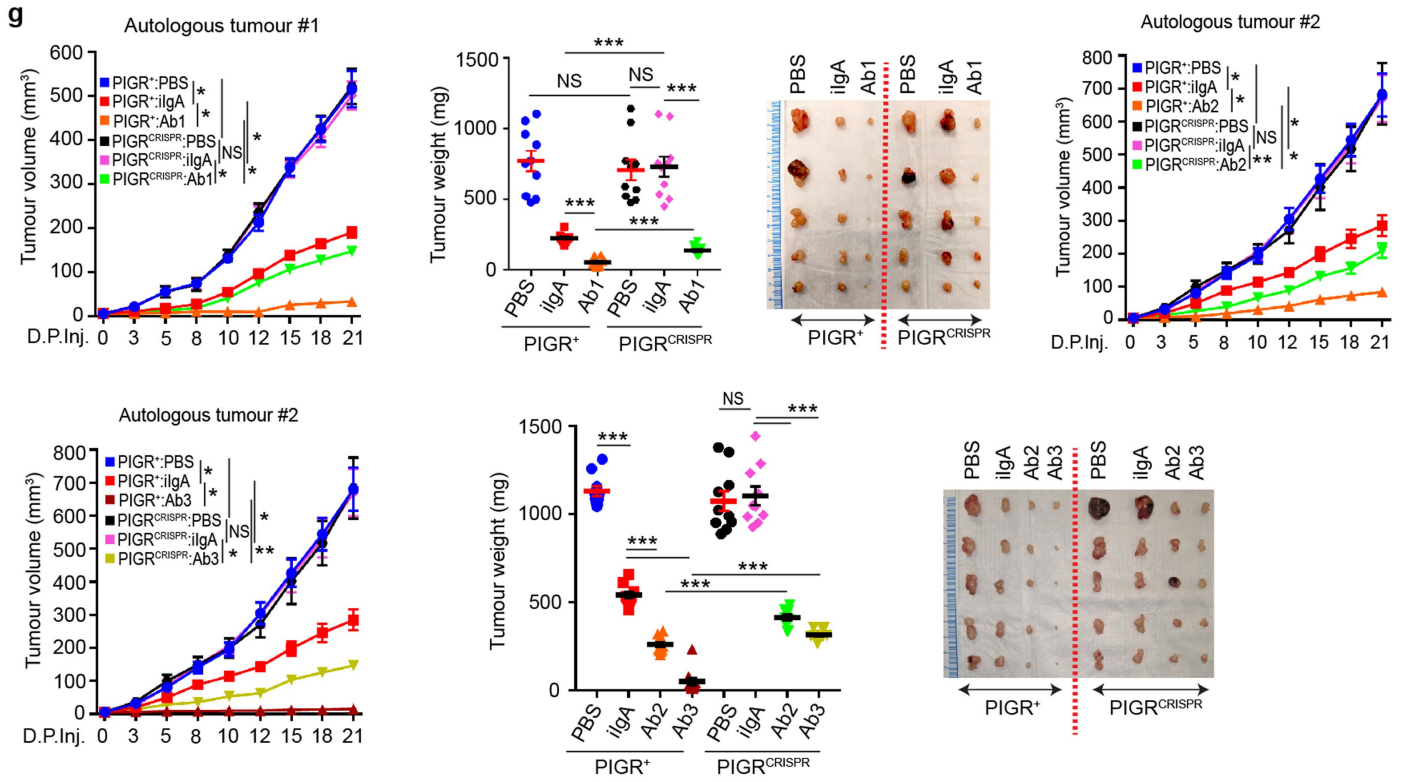

Extended Data Fig. 6 | See next page for caption. 


\section{Article}

Extended Data Fig. 6 | Tumour-derived IgA abrogates tumour growth through antigen-dependent redirection of $F \mathrm{c} \alpha / \boldsymbol{\mu} \mathbf{R}^{+}$myeloid cells and antigen-independent, pIgR-mediated transcytosis. a, Dot plots showing FACS analysis of splenocytes for NK1.1 depletion in RAG1-KO mice (left). Scatter plot showing $\mathrm{CD} 45^{+} \mathrm{NK} 1.1^{+}$cells percentages among viable splenocytes in respective treatment-group mice $(n=10$ mice per group pooled from 2 independent experiments) (right). $P<0.0001$, ordinary one-way ANOVA. Data are mean \pm s.e.m. ${ }^{* * *} P \leq 0.001$. b, Representative FACS dot plots showing binding of IgA antibodies to splenic $\mathrm{CD} 11 \mathrm{~b}^{+}$cells from tumour-bearing RAG1deficient mice, after incubation with $\mathrm{Fc} \alpha / \mu \mathrm{R}(\mathrm{CD} 351)$-neutralizing antibodies or isotype controls $(n=10)$. c, Representative FACS dot plots show CD $351^{+}$cells among viable $\mathrm{CD} 45^{+} \mathrm{CD} 11 \mathrm{~b}^{+}$cells in xenografts in RAG1-deficient mice treated with intratumoural anti-TSPAN7 (with or without NK1.1-depletion), compared to irrelevant IgA or vehicle (PBS) ( $n=10$ mice per group pooled from 2 independent experiments). d, Tumour growth curves (left), as well as tumour volume (right) and weight (centre) in pIgR ${ }^{\mathrm{CRISPR}}$ OVCAR3-tumour-bearing NSG mice receiving irrelevant IgA antibodies or vehicle (PBS). Paired or unpaired two-tailed $t$-tests for tumour growth curves or tumour weight comparisons, respectively. Data are mean \pm s.e.m. NS, not significant. e, Internalized intensity of antibodies (APC) were quantified and scattered bar graph showing comparison of antibody internalization in different treatment conditions, in which each dot represents quantification from one cell, pooled from three independent experiments. Supplementary Table 11 provides details of statistics. Data are mean \pm s.e.m. ${ }^{* * *} P \leq 0.001$, NS, not significant; unpaired twotailed $t$-test.f, Pathway analysis of RNA-seq from OVCAR3 cells treated with irrelevant IgA $\left(0.5 \mu \mathrm{g} \mathrm{ml}^{-1}\right)$, anti-TSPAN7-IgA $\left(0.5 \mu \mathrm{g} \mathrm{ml}^{-1}\right)$, anti-BDNF-IgA or no treatment for $24 \mathrm{~h}(n=3)$. g, Autologous tumour growth curves, tumour weight and volume in wild-type $\operatorname{pIgR}^{+}(\mathrm{WT})$ or $P I G R$-ablated (pIgR $\left.{ }^{\mathrm{CRISPR}}\right)$ autologous tumour-bearing RAG1-deficient mice receiving control or recombinant dimeric IgA antibodies (Ab1, Ab2 or Ab3) produced with three different matching IgA sequences clonally expanded in two different HGSOCs. Respective autologous HGSOC cells were used (tumour no. 1 for $A b 1$ and tumour no. 2 for $A b 2$ or Ab3). Data are mean \pm s.e.m. ${ }^{*} P \leq 0.05,{ }^{* *} P \leq 0.01,{ }^{* * *} P \leq 0.001$, NS, not significant. Supplementary Table 12 provides details of statistics. In $\mathbf{d}, \mathbf{g}$, curves and tumour weights were pooled from 2 independent experiments $(n=10$ mice per group in total). 
$\mathbf{a}_{\text {Tonsil }}$

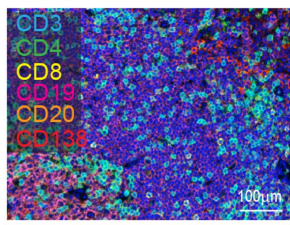

DAPI only
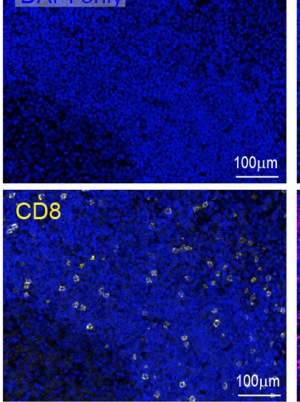

CD8 Isotype Control

$100 \mu \mathrm{m}$

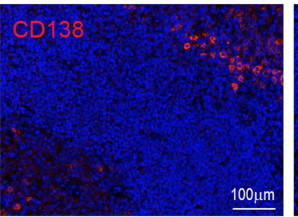

$100 \mu \mathrm{m}$
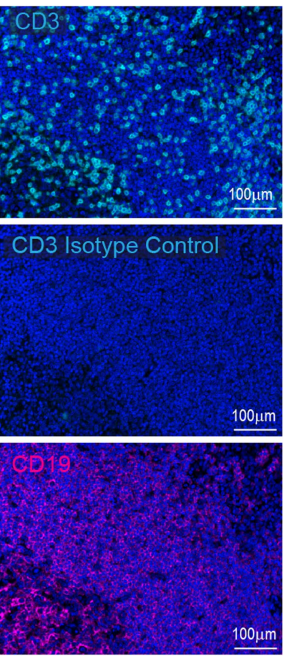

CD19 Isotype Control
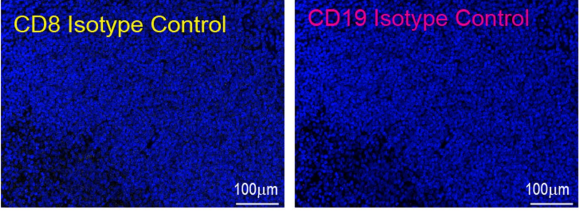

5
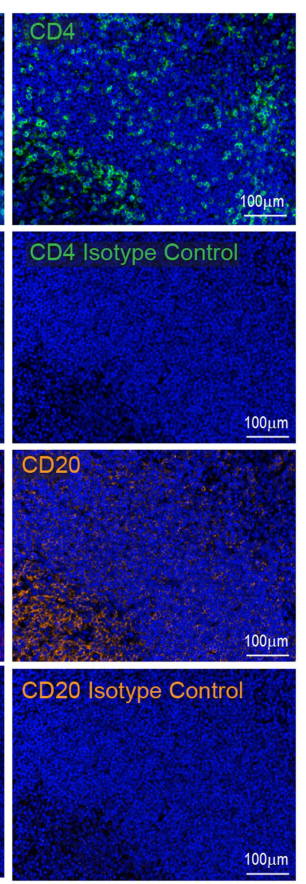

b Tonsil

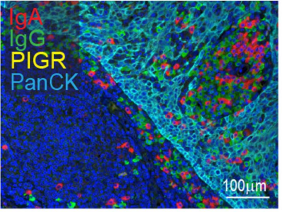

DAPI only
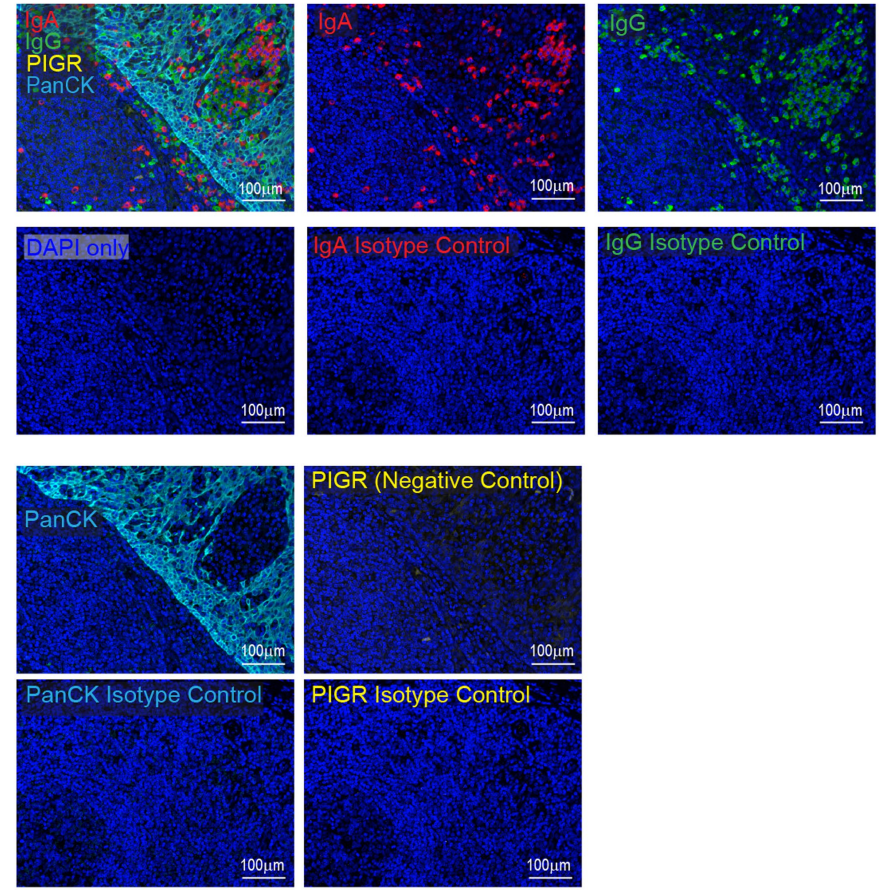

PIGR (Negative Control)
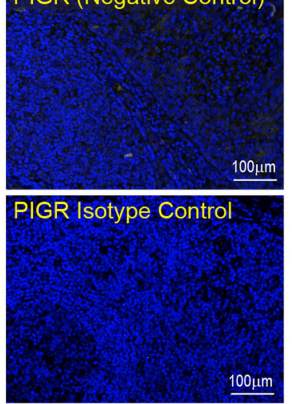

Extended Data Fig. $7 \mid$ Multiplex immunohistochemistry staining optimization for CD3, CD4, CD8, CD19, CD20, CD138, IgA, IgG, pIgR and PCK in tonsil tissues. a, $\mathbf{b}$, Multiplex staining of tonsil tissue sections $(n=5)$ with anti-human CD3, CD4, CD8, CD19, CD20 and CD138 antibodies or respective isotype controls (a), and IgA, IgG, plgR and PCK antibodies or respective isotype controls (b). Scale bar, $100 \mu \mathrm{m}$. 


\section{Article}

a
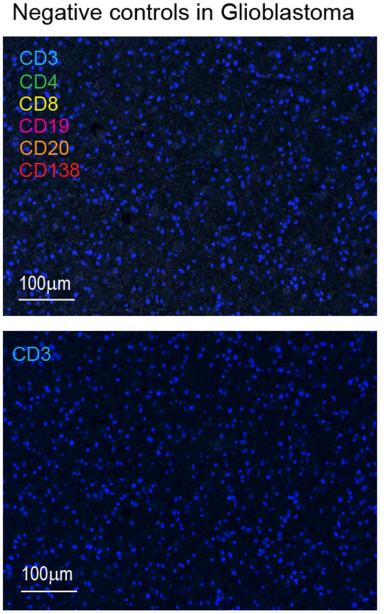

CD4.

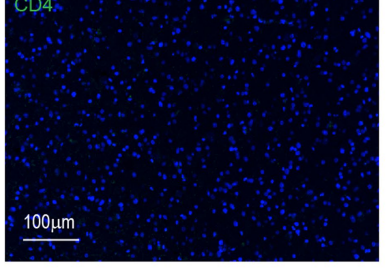

CD8

$100 \mu \mathrm{m}$
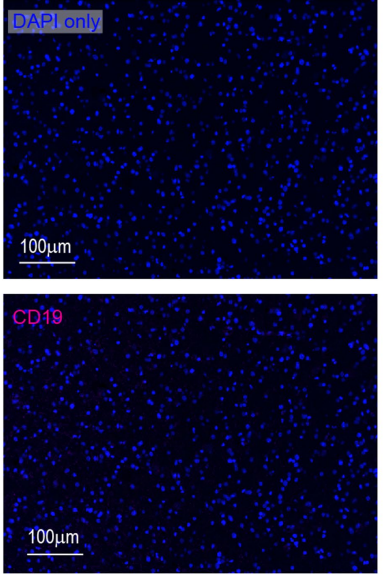

CD20

$100 \mu \mathrm{m}$

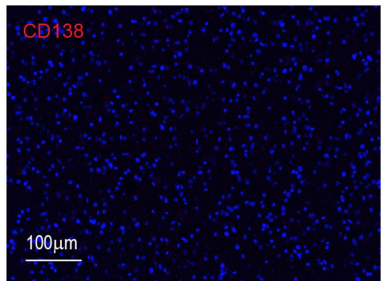

Negative controls in Glioblastoma
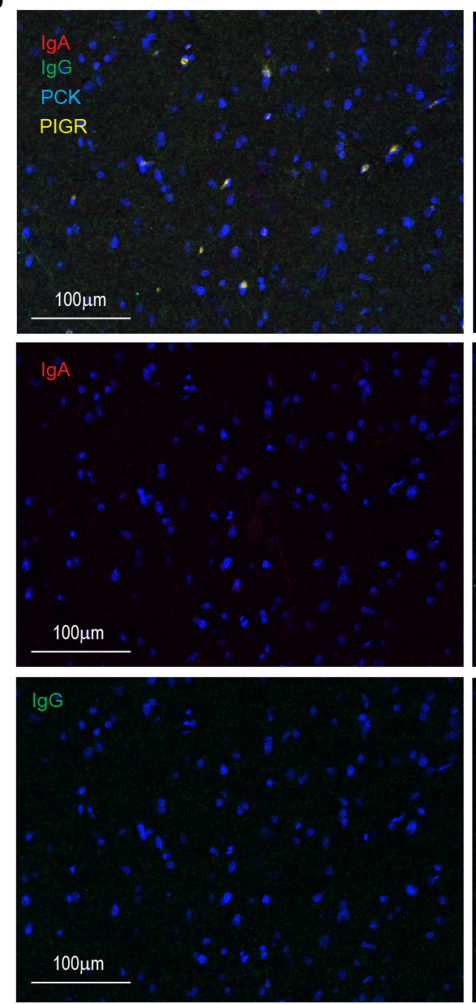

Kidney

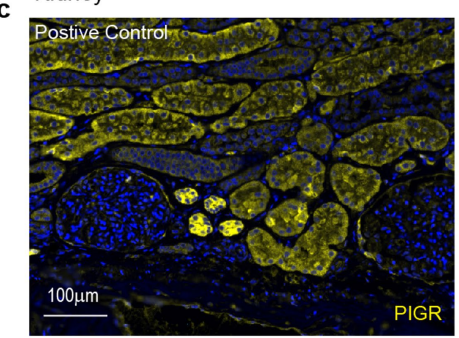

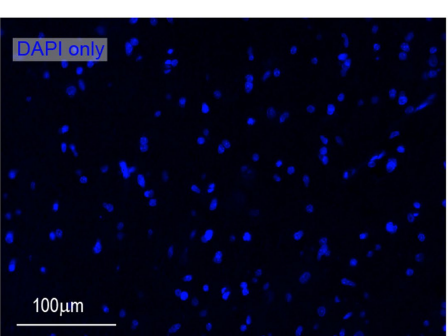

PCK
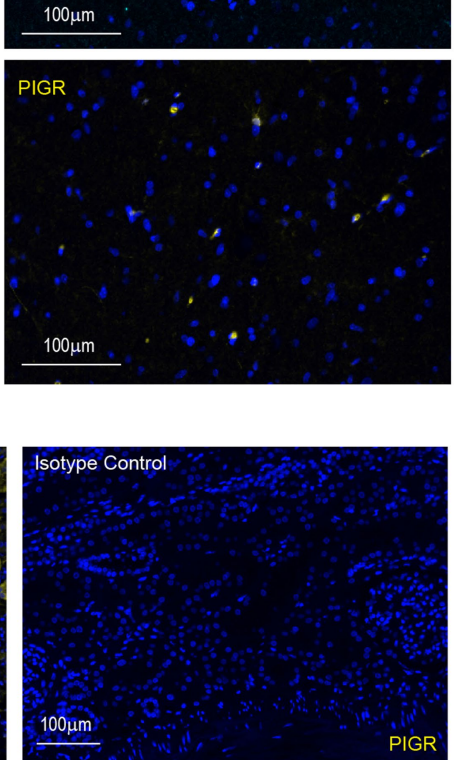

CD19, CD20 and CD138 antibodies (a), and IgA, IgG, pIgR and PCK antibodies (b). Scale bar, $100 \mu \mathrm{m}$.c, Multiplex staining of healthy kidney tissue sections $(n=4)$ with anti-human plgR antibody and respective isotype control. Scale bar, $100 \mu \mathrm{m}$. optimization for CD3, CD4, CD8, CD19, CD20, CD138, IgA, IgG, plgR and PCK in glioblastoma tissues, and for pIgR in kidney tissues. a, b, Multiplex staining of glioblastoma tissue sections $(n=5)$ with anti-human CD3, CD4, CD8, 

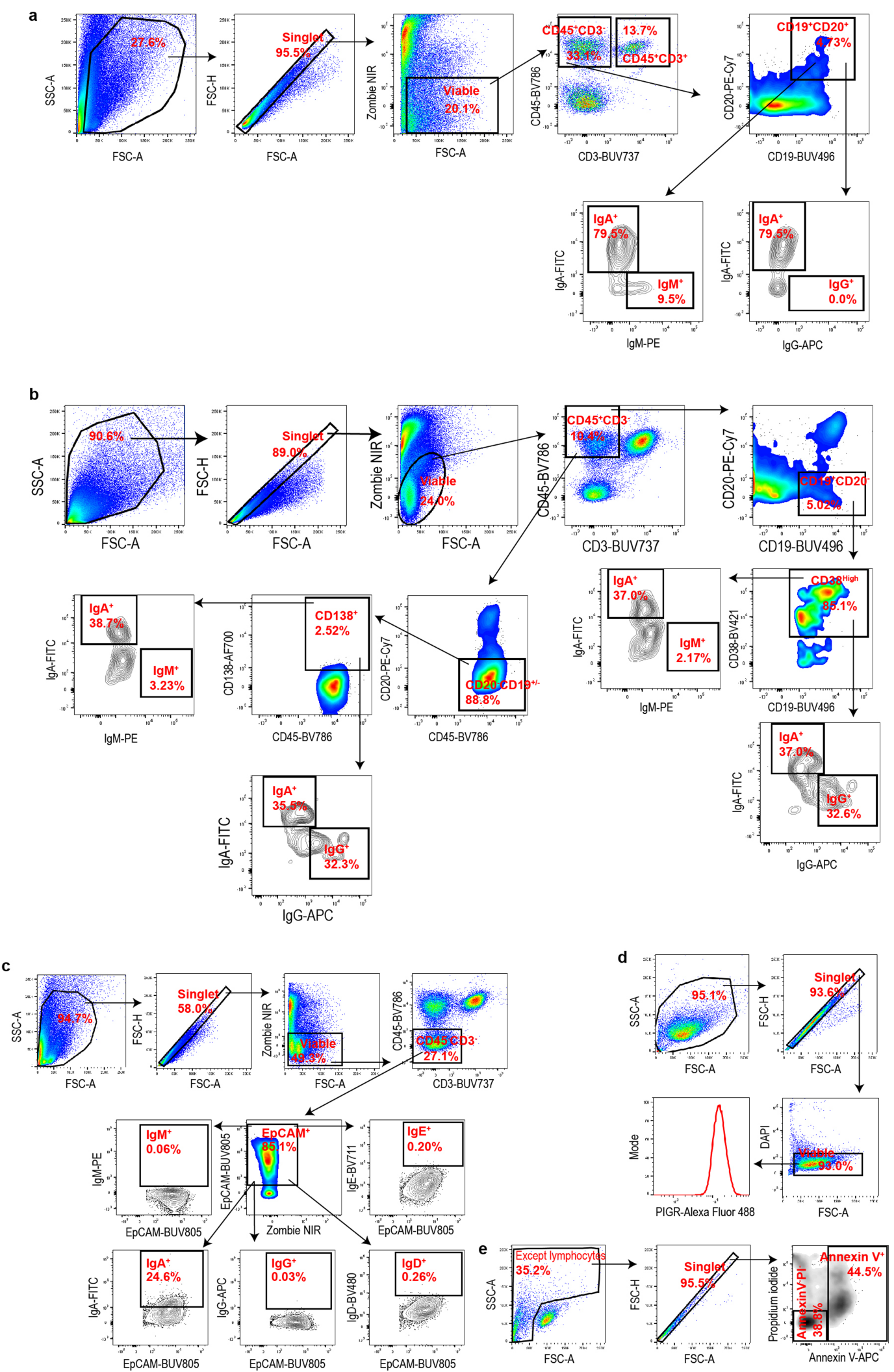

Extended Data Fig. 9 | Flow cytometry gating strategies. a, b, Gating strategies for FACS analysis shown in Fig. 1a, Extended Data Figs.1g, h, i, 2a, d, e. c, Gating strategies for FACS analysis shown in Fig. 1b. d, Gating strategies for
FACS analysis shown in Fig. 1d. e, Gating strategies for FACS analysis shown in Fig. 1g, Extended Data Fig. 4j, k. 

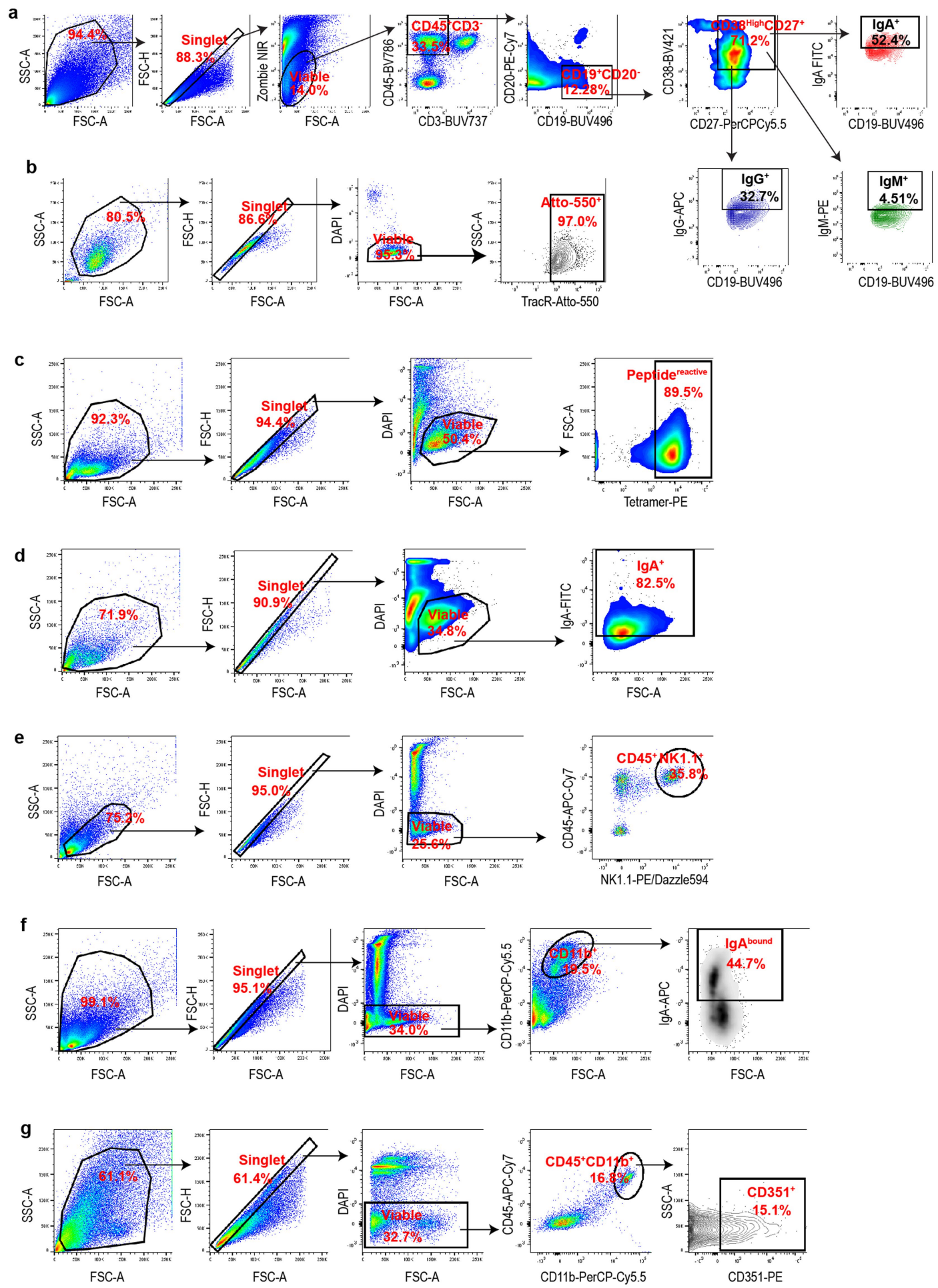

Extended Data Fig. 10 | Further flow cytometry gating strategies. a, Gating strategies for FACS analysis shown in Extended Data Fig. 2a.b, Gating strategies for FACS analysis shown in Extended Data Fig. 3c.c, Gating strategies for FACS analysis shown in Extended Data Fig. 5f. d, Gating strategies for FACS analysis shown in Extended Data Fig. 5g. e, Gating strategies for FACS analysis shown in Extended Data Fig. 6a.f, Gating strategies for FACS analysis shown in Extended Data Fig. 6b. g, Gating strategies for FACS analysis shown in Extended Data Fig. 6c. 


\section{Reporting Summary}

Nature Research wishes to improve the reproducibility of the work that we publish. This form provides structure for consistency and transparency in reporting. For further information on Nature Research policies, see our Editorial Policies and the Editorial Policy Checklist.

\section{Statistics}

For all statistical analyses, confirm that the following items are present in the figure legend, table legend, main text, or Methods section.

$\mathrm{n} / \mathrm{a} \mid$ Confirmed

$\bigotimes$ The exact sample size $(n)$ for each experimental group/condition, given as a discrete number and unit of measurement

$\bigotimes$ A statement on whether measurements were taken from distinct samples or whether the same sample was measured repeatedly

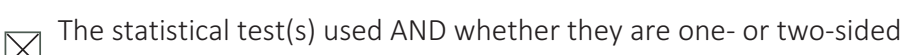

Only common tests should be described solely by name; describe more complex techniques in the Methods section.

$\bigotimes$ A description of all covariates tested

$\bigotimes$ A description of any assumptions or corrections, such as tests of normality and adjustment for multiple comparisons

A full description of the statistical parameters including central tendency (e.g. means) or other basic estimates (e.g. regression coefficient)

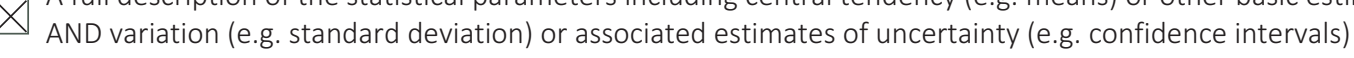

$\varnothing$ For null hypothesis testing, the test statistic (e.g. $F, t, r$ ) with confidence intervals, effect sizes, degrees of freedom and $P$ value noted

Give $P$ values as exact values whenever suitable.

\ $\square$ For Bayesian analysis, information on the choice of priors and Markov chain Monte Carlo settings

\ $\square$ For hierarchical and complex designs, identification of the appropriate level for tests and full reporting of outcomes

$\bigotimes$ Estimates of effect sizes (e.g. Cohen's $d$, Pearson's $r$ ), indicating how they were calculated

Our web collection on statistics for biologists contains articles on many of the points above.

\section{Software and code}

Policy information about availability of computer code

Data collection FACS data was collected from BD FACS LSR II and BD FACS ARIA using BD FACS Diva v8.0.1. Multiplex data was collected from PerkinEImer Vectra ${ }^{\oplus} 3$ Automated Quantitative Pathology Imaging System inForm v2.4.8. RNA Sequencing reads were collected using bcl2fastq v2.20. Confocal microscopy image acquisition was performed and data collected from Leica SP8 using LAS X (v3.5.5.19976). For quantitative analysis, fluorescence image acquisition was performed in Zeiss Imager Z2 upright microscope and data was collected using ZEN 2.3 (blue edition) software. H/E histology slides were scanned in Aperio-Leica Scanner Console (v102.0.7.5) and data were collected.

Data analysis All statistical tests were run using Graphpad Prism (v7.0) or R (v3.6.1). Flow cytometry data was analyzed with FlowJo v10.7.1. Dot plots from Multiplex images were done using FCS Image v7.0. Multiplex images were analyzed in inForm (v2.4.8) and HALO (v3.0.311.328). RNA-seq sequence processing and statistics was performed using cutadapt (v1.8.1), STAR (v2.5.3a), featureCounts (v1.5.3), htseq-count (v0.6.1), and DESeq2 (v1.30.0). GSEA (v4.0.2) was performed using gene sets from the MSigDB database. Single-cell V(D)J (BCR) sequence data was analyzed using CellRanger V(D)J (v3.1.0). RNA-seq and single-cell data visualization was performed in R (v3.6.1). CZI image files were imported into Definiens Tissue Studio (v4.7) and analyzed. 
Policy information about availability of data

All manuscripts must include a data availability statement. This statement should provide the following information, where applicable:

- Accession codes, unique identifiers, or web links for publicly available datasets

- A list of figures that have associated raw data

- A description of any restrictions on data availability

The RNA sequencing data and single cell BCR sequencing data related to this study are available at the NCBI Gene Expression Omnibus (GEO) under accession number GSE146820. The mass spectrometry proteomics data are available in PRIDE with identifier PXD018079. Source data are provided with this paper. Molecular and clinical data from The Cancer Genome Atlas for Ovarian Serous Cystadenocarcinoma (OV) are available at the cBio Cancer Genomics Portal (http:// www.cbioportal.org/), Broad Firehose website (https://gdac.broadinstitute.org/), and Genomic Data Commons Data Portal (https://portal.gdc.cancer.gov/). Source data are provided with this paper. The datasets generated during the current study are available from the corresponding author upon reasonable request.

\section{Field-specific reporting}

Please select the one below that is the best fit for your research. If you are not sure, read the appropriate sections before making your selection. \Life sciences Behavioural \& social sciences Ecological, evolutionary \& environmental sciences

For a reference copy of the document with all sections, see nature.com/documents/nr-reporting-summary-flat.pdf

\section{Life sciences study design}

All studies must disclose on these points even when the disclosure is negative.

Sample size Sample size for every experiment has been described in the manuscript and is based on the availability of adequate samples. No sample size calculations were performed prior to the study for human specimens. For most functional in vitro analyses, sample sizes were chosen based on the availability of target cells. Animal experiments used at least five mice per group per experiments. Since this study focuses on ovarian cancer, only female mice were included in the experimental design.

Data exclusions No data were excluded

Replication Experiments were performed at least two times and/or with sufficient cells/animals per group to demonstrate statistical significance.

Randomization The experiments were not randomized.

-HGSOC tumour and ascites specimens were obtained from de-identified patients and were not randomized. PBMC from de-identified donors without cancer were acquired and analyzed.

- Animals were not intentionally randomized. All animal experiments were conducted using 4-6 week-old female RAG1-deficient or NSG mice with procured from Charles River Laboratories and Jackson Laboratory, respectively.

Blinding $\quad$-Tumour volumes in mice were measured using code names on the cages and ear tags, instead of specific information about the treatments that the animals received. Apart from this, no blinding method was used for animal studies.

-RNA sequencing, BCR sequencing, Multiplex immunohistochemistry quantifications, fluorescence microscopy quantifications or LC-MS/MS were performed with unidentifiable demarcation.

-In case of in vitro experiments, samples often assigned code numbers to facilitate blinded flow cytometry, microscopy, luciferase assay. After all data were collected, the results were analyzed and decoded.

-For analysis of human specimens blinding is not applicable as no interventions were tested.

\section{Reporting for specific materials, systems and methods}

We require information from authors about some types of materials, experimental systems and methods used in many studies. Here, indicate whether each material, system or method listed is relevant to your study. If you are not sure if a list item applies to your research, read the appropriate section before selecting a response.

Materials \& experimental systems

Methods

Involved in the study

$\bigotimes$ Antibodies

$\bigotimes$ Eukaryotic cell lines

$\square$ Palaeontology and archaeology $\mathrm{n} / \mathrm{a}$ Involved in the study

Х $\square$ ChIP-seq

$\square \bigotimes$ Flow cytometry

$\bigotimes$ Animals and other organisms

\ $\square$ MRI-based neuroimaging

$\square$ Human research participants

\ $\square$ Clinical data

$\bigotimes \square$ Dual use research of concern 

anti-IgG-APC, BioLegend, 410720, M1310G05, Lot-B243888; anti-IgM-PE, BioLegend, 314508, MHM-88, Lot-B235317; anti-IgD-BV480, BD Biosciences, 566138, IA6-2, Lot-6294773; anti-IgE-BV711, BD Biosciences, 744319, G7-26, Lot-8002780; anti-plgR, ThermoScientific, PA5-35340, Lot-VE3000758; anti-plgR, Abcam, ab96196, Lot-GR3263410-2; anti-Phospho-Erk1/2, CST, 5726, D1H6G, Lot-1; anti-Erk1/2, CST, 4696, L34F12, Lot-23;

anti-DUSP5, Abcam, ab200708, EPR19684, Lot-GR3186774-2; anti- $\beta$-actin, CST, 4970, 13E5, Lot-15 and CST, 5125, 13E5, Lot-6; anti-IgA, Abcam, ab124716, EPR5367-76, Lot-GR87907-13; anti-IgG, Abcam, ab109489, EPR4421, Lot-GR3258727-4; anti-plgR-Secretory component, Abcam, ab3924, SC-05, GR3299752-2; anti-CD3, Dako, A0452, Lot-20057103; anti-CD4, Cell Marque, 104R-25, EP204, Lot- 53921; anti-CD8, Dako, M7103, C8/144B, Lot- 20055137; anti-CD19, Dako, M7296, LE-CD19, Lot-20067725 anti-CD20, Dako, M0755, L26, Lot-20042864; anti-CD138, Dako, M7228, MI15, Lot-20050508; anti-Pan Cytokeratin, Dako, M3515, AE1/AE3, Lot-10149487;

anti-mouse antibodies: anti-CD45-APC-Cy7, BioLegend, 103116, 30-F11, Lot-B257634; anti-CD11b-PerCP-Cy5.5, BioLegend, 101228, M1/70, Lot-B247471; anti-CD351-PE, BioLegend, 137306, TX61, Lot-B265236 anti-CD351, BioLegend, 137303, TX61, Lot- B275404; anti-NK1.1-PE/Dazzle594, BioLegend, 108748, PK136, Lot- B213746; anti-NK1.1, BioXCell, BE0036, PK136, Lot-693118J3;

Human native antibodies:

Native Human IgA, Abcam, ab91025, Lot-GR3246098-6;

Native Human IgG, Abcam, ab98981, Lot-GR3269929-2

Antibodies used in flow cytometry- Most of the primary antibodies used for flow cytometry in this study are widely used and well validated. The mentioned antibodies are tested by flow cytometry analysis by the manufacturer and/or in our lab: anti-CD45- staining of human peripheral blood lymphocytes by flow cytometry anti-CD3- staining of human peripheral blood lymphocytes by flow cytometry anti-CD19- staining of human peripheral blood leucocytes by flow cytometry anti-CD20- staining of human peripheral blood lymphocytes by flow cytometry anti-CD38- staining of human peripheral blood lymphocytes by flow cytometry anti-CD138- staining of human myeloma cell line U266 by flow cytometry anti-CD27- staining of human peripheral blood lymphocytes by flow cytometry anti-EpCAM- staining of human OVCAR3 cell line by flow cytometry anti-IgA- staining of human peripheral blood lymphocytes flow cytometry anti-lgG- staining of human peripheral blood lymphocytes by flow cytometry anti-IgM- staining of by human peripheral blood lymphocytes flow cytometry anti-lgD- staining of by human peripheral blood lymphocytes flow cytometry anti-lgE- staining of human peripheral blood lymphocytes by flow cytometry anti-plgR- staining of human HepG2 cell line by flow cytometry anti-CD45- staining of C57BL/6 mouse splenocytes by flow cytometry anti-CD11b- staining of C57BL/6 mouse splenocytes by flow cytometry anti-CD351- staining of Con-A stimulated C57BL/6 splenocytes by flow cytometry anti-NK-1.1- staining of C57BL/6 mouse splenocytes by flow cytometry

Antibodies used in in vitro or in vivo neutralization- We used following neutralizing antibodies with relevant publication references available at the manufacturer's website:

anti-CD351- block interaction with the Fca/ $\mu$ receptor (ref: Shibuya A, et al., Nat Immunol. 2000 Nov;1(5):441-6. doi: 10.1038/80886. PMID: 11062505)

anti-NK-1.1- in vivo depletion of NK1.1 expressing cells, mice (ref: Glasner A, et al., Immunity. 2018 Jan 16;48(1):107-119.e4. doi: 
Antibodies used in western blot and IP- Most of the primary antibodies used for western blotting and immunoprecipitation in this study are widely used and well validated. The mentioned antibodies are tested by the manufacturer and/or in our lab: anti-plgR- staining of Molt4 whole cell lysate by western blot analysis anti-IgA- staining of lysates from human tonsil, plasma and spleen by western blot analysis; Immunoprecipitation of IgA from human plasma and developed by western blot analysis

anti-phospho-Erk1/2- staining of extracts from A-431, HeLA, C6, COS-7 cell lines by western blot analysis anti-Erk1/2- staining of extracts from NIH/3T3, PC12 and COS cells by western blot analysis anti-DUSP5- staining of MEF (Mouse embryonic fibroblast cell line) whole cell lysate by western blot analysis anti- $\beta$-actin- staining of extracts from ACTA1, ACTA2, ACTB, ACTC, ACTG1, ACTG2, HeLa cell lines by western blot analysis anti- $\beta$-actin- staining of extracts from NIH/3T3, HeLa, COS, PC12 cell lines by western blot analysis anti-IgA- secretory component staining of MCF7 and MDA-MB-361 whole cell lysates by western blot analysis

Antibodies used in multiplex immunohistochemistry- Most of the primary antibodies used for multiplex immunohistochemistry in this study are widely used and well validated. The mentioned antibodies are tested by immunohistochemical analysis by the manufacturer and/or in our core facility:

anti-CD3- staining of sections of human tonsil tissues by immunohistochemistry anti-CD4- staining of sections of human tonsil and lymph node tissues by immunohistochemistry anti-CD8- staining of sections of human tonsil and spleen tissues by immunohistochemistry anti-CD19- staining of sections of human lymphoma, tonsil, precursor B-cell lymphoblastic leukemia/lymphoma, B-cell chronic lymphocytic leukemia/small lymphocytic lymphoma tissues by immunohistochemistry anti-CD20- staining of sections of human tonsil, mantle cell lymphoma, B-cell chronic lymphocytic leukemia/small lymphocytic lymphoma tissues by immunohistochemistry anti-CD138- staining of sections of human high-grade myeloma, tonsil, large B-cell lymphoma and appendix by immunohistochemistry

anti-pan-cytokeratin- staining of sections of human tonsil, seminoma, liver, merkel cell tumour tissues by immunohistochemistry anti-plgR- staining of sections of Cal27 xenograft tissue and HepG2 cells by immunohistochemistry anti-IgA- staining of sections of human colon and tonsil tissues by Immunohistochemistry anti-IgG- staining of sections of human tonsil tissues by Immunohistochemistry

\section{Eukaryotic cell lines}

Policy information about cell lines

Cell line source(s)

Authentication

Mycoplasma contamination

Commonly misidentified lines (See ICLAC register)
OVCAR3, A549, NIH-H23, HEK-293T, K562, THP1 cells were purchased from ATCC (Manassas, VA); OVCAR4, OVCAR5 and OVACR8 cells were procured from National Cancer Institute (Bethesda, MD); Kuramochi cell line was procured from JCRB Cell Bank, Japan. Human Ovarian Surface Epithelial (OSE) Cells were purchased from ScienCell Research Laboratories.

Cell lines were not authenticated

Cell lines were routinely tested negative for mycoplasma contamination

None

\section{Animals and other organisms}

Policy information about studies involving animals; ARRIVE guidelines recommended for reporting animal research

Laboratory animals

Female, 4-6 weeks old RAG1-deficient (B6.129S7-RAG1 knock-out) mice and NSG mice of same age groups were procured from Charles River Laboratories and Jackson Laboratory, respectively

Wild animals

None

Field-collected samples None

Ethics oversight

Institutional Animal Care and Use Committee at the University of South Florida

Note that full information on the approval of the study protocol must also be provided in the manuscript.

\section{Human research participants}

Policy information about studies involving human research participants

Population characteristics

-Human high grade serous ovarian cancer (HGSOC) tissues were procured under protocols approved by the Committee for the Protection of Human Subjects at Dartmouth-Hitchcock Medical Center, and under a protocol approved by H. Lee Moffitt Cancer Center. All specimens were classified as surgical discard and remained totally de-identified. Stage III-IV human HGSOC 
specimens and malignant ascites samples were procured. Use of samples in MCC cohort TMA was approved by institutional review board at Moffitt Cancer Center. Use of samples in the NHS cohort was approved by institutional review board at Brigham and Women's Hospital and Harvard T.H. Chan School of Public Health. Use of samples in NECC cohort TMA was approved by institutional review boards at Brigham and Women's Hospital and Dartmouth Medical School. Universal consent form was obtained for all subjects. All HGSOC specimens analyzed in this study are described in Supplementary Table 1.

- We obtained randomly selected peripheral blood mononuclear cells (PBMCs) from de-identified, cancer-free female donors (Moffitt Cancer Center).

Recruitment

Ethics oversight
None

Moffitt Cancer Center Institutional Review Board

Note that full information on the approval of the study protocol must also be provided in the manuscript.

\section{Flow Cytometry}

\section{Plots}

Confirm that:

\The axis labels state the marker and fluorochrome used (e.g. CD4-FITC).

\The axis scales are clearly visible. Include numbers along axes only for bottom left plot of group (a 'group' is an analysis of identical markers).

$\bigotimes$ All plots are contour plots with outliers or pseudocolor plots.

\A numerical value for number of cells or percentage (with statistics) is provided.

\section{Methodology}

Sample preparation

Instrument

Software

Cell population abundance

Gating strategy
Flow cytometry was performed by staining with Zombie NIR (BioLegend) or Zombie Yellow (BioLegend) or DAPI (ThermoScientific) viability dye, blocking with anti-CD16/32 (BioLegend), and staining for $30 \mathrm{~min}$ at 4 으 with the following anti-human antibodies: CD45 (BD Biosciences, HI30, 1:300), CD3 (BD Biosciences, SK7, 1:200), CD19 (BD Biosciences, HIB19, 1:200), CD20 (BioLegend, 2H7, 1:200), CD38 (BD Biosciences, HIT2, 1:200), CD138 (BioLegend, MI15, 1:200), CD27 (BD Biosciences, M-T271, 1:200), IgA (Tonbo Biosciences, 35-8016-M001, 1:20), IgG (BioLegend, M1310G05, 1:200), IgM (BioLegend, MHM-88, 1:200), IgD (BD Biosciences, IA6-2, 1:100), IgE (BD Biosciences, G7-26, 1:100), EpCAM (BD Biosciences, KS1/4, 1:200), plgR (ThermoScientific, PA5-35340, 1:50) or tetramers against TSPAN7 or BDNF. For intracellular staining for immunoglobulin isotypes, cells were first incubated with surface staining antibodies (30 min in ice), followed by fixation (30 min in RT) (eBioscience) and finally incubation with the antibodies in the permeabilization buffer (eBioscience) with antibodies for intracellular markers (45 min in RT).

Mice xenograft tumour single cell suspensions or splenocytes were blocked with Fc blocker (BioLegend) and analyzed by flow cytometry after incubation for $30 \mathrm{~min}$ at 4 으 with following anti-mouse antibodies: CD45 (BioLegend, 30-F11), CD11b (BioLegend, M1/70), CD351 (BioLegend, TX61) or with APC-conjugated human IgA. Splenocytes from RAG1-deficient mice were mechanically dissociated and RBCs were removed, followed by neutralization of $F c \alpha / \mu$ receptor (Fc $\alpha / \mu R$ ) by incubation with CD351-neutralizing antibodies (BioLegend, TX61, 137303) or with isotype controls (BioLegend, 400123) at a concentration of $2.0 \mu \mathrm{g} / 106$ cells in $100 \mu \mathrm{l}$ volume for $30 \mathrm{~min}$ in ice. After washing, splenocytes were then incubated with APC-conjugated human IgA for another $30 \mathrm{~min}$ in ice and analyzed by flow cytometry.

FACS LSR-II and FACS Aria sorter, BD Biosciences

FACS Diva and FlowJo_V10

Post-sort purity was analysed for samples with more than 5000 target cells collected. In all cases purity was greater than $95 \%$

Gating strategy is shown in Extended Data Figures 26-29

$\triangle$ Tick this box to confirm that a figure exemplifying the gating strategy is provided in the Supplementary Information. 